\title{
Prandtl boundary layer expansions of steady Navier-Stokes flows over a moving plate
}

\author{
Yan Guo* Toan T. Nguyen ${ }^{\dagger}$
}

April 3, 2018

\begin{abstract}
This paper concerns the validity of the Prandtl boundary layer theory in the inviscid limit for steady incompressible Navier-Stokes flows. The stationary flows, with small viscosity, are considered on $[0, L] \times \mathbb{R}_{+}$, assuming a no-slip boundary condition over a moving plate at $y=0$. We establish the validity of the Prandtl boundary layer expansion and its error estimates.
\end{abstract}

\section{Contents}

1 Introduction $\quad 2$

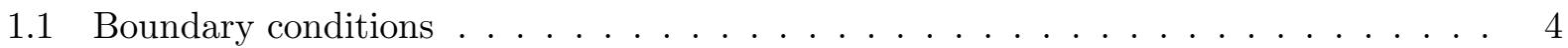

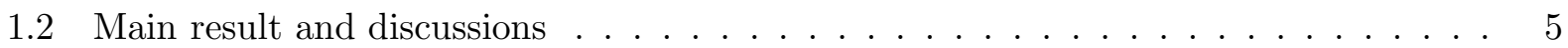

2 Construction of the approximate solutions 8

2.1 Zeroth-order Prandtl layers . . . . . . . . . . . . . . . . . . . . 9

$2.2 \varepsilon^{1 / 2}$-order corrections . . . . . . . . . . . . . . . . . . . . 13

2.3 Euler correctors . . . . . . . . . . . . . . . . . . . . . . 14

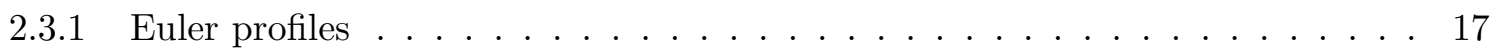

2.4 Prandtl correctors . . . . . . . . . . . . . . . . . . . . 18

2.4.1 Construction of Prandtl layers . . . . . . . . . . . . . . . . 19

2.4.2 Cut-off Prandtl layers . . . . . . . . . . . . . . . . . . . 25

2.5 Proof of Proposition $2.1 \ldots \ldots \ldots \ldots \ldots \ldots \ldots \ldots$

3 Linear stability estimates 2

3.1 Energy estimates . . . . . . . . . . . . . . . . . . . . . . . . . 29

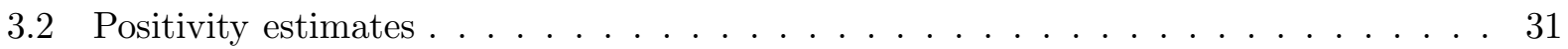

3.3 Proof of Proposition $3.1 \ldots \ldots \ldots \ldots \ldots$

$4 \quad L^{\infty}$ estimates $\quad 38$

5 Proof of the main theorem

*Division of Applied Mathematics, Brown University, 182 George street, Providence, RI 02912, USA. Email: Yan_Guo@Brown.edu

${ }^{\dagger}$ Department of Mathematics, Pennsylvania State University, State College, PA 16802, USA. Email: nguyen@math.psu.edu. 


\section{Introduction}

In this paper, we consider the stationary incompressible Navier-Stokes equations

$$
\begin{aligned}
U U_{X}+V U_{Y}+P_{X} & =\varepsilon U_{X X}+\varepsilon U_{Y Y} \\
U V_{X}+V V_{Y}+P_{Y} & =\varepsilon V_{X X}+\varepsilon V_{Y Y} \\
U_{X}+V_{Y} & =0
\end{aligned}
$$

posed in a two dimensional domain $\Omega=\{(X, Y): 0 \leq X \leq L, Y \geq 0\}$, with a "no-slip" boundary condition

$$
U(X, 0)=u_{b}>0, \quad V(X, 0)=0
$$

on the boundary at $Y=0$. The given constant $u_{b}$ can be viewed as the moving speed of the plate (that is, the boundary edge $Y=0$ ). The case when $u_{b} \equiv 0$ refers to the classical no-slip boundary condition on a motionless boundary $Y=0$. The boundary conditions at $x=0, L$ will be prescribed explicitly in the text.

We are interested in the problem when $\varepsilon \rightarrow 0$. The study of the inviscid limit and asymptotic boundary layer expansions of Navier-Stokes equations (1.1) in the presence of no-slip boundary condition is one of the central problems in the mathematical analysis of fluid mechanics. A formal limit $\varepsilon \rightarrow 0$ should lead the Euler flow $\left[U_{0}, V_{0}\right]$ inside $\Omega$ which satisfies only non-penetration condition at $Y=0$ :

$$
V_{0}(X, 0)=0 .
$$

Throughout this paper, we assume that the outside Euler flow is a shear flow

$$
\left[U_{0}, V_{0}\right] \equiv\left[u_{e}^{0}(Y), 0\right]
$$

for some smooth functions $u_{e}^{0}(Y)$. We note that there is no pressure $p_{e}$ for this Euler flow. Generically, there is a mismatch between the tangential velocities of the Euler flow $u_{e} \equiv U_{0}(X, 0) \equiv u_{e}^{0}(0)$ and the prescribed Navier-Stokes flows $U(X, 0)=u_{b}$ on the boundary.

Due to the mismatch on the boundary, Prandtl in 1904 proposed a thin fluid boundary layer of size $\sqrt{\varepsilon}$ to connect different velocities $u_{e}$ and $u_{b}$. We shall work with the scaled boundary layer, or Prandtl's, variables:

$$
x=X, \quad y=\frac{Y}{\sqrt{\varepsilon}} .
$$

In these variables, we express the solution of the NS equation $[U, V]$ via $\left[U^{\varepsilon}, V^{\varepsilon}\right]$ as

$$
[U(X, Y), V(X, Y)]=\left[U(x, \sqrt{\varepsilon} y), \sqrt{\varepsilon}\left\{\frac{V(x, \sqrt{\varepsilon} y)}{\sqrt{\varepsilon}}\right\}\right]=\left[U^{\varepsilon}(x, y), \sqrt{\varepsilon} V^{\varepsilon}(x, y)\right]
$$

in which we note that the scaled normal velocity $V^{\varepsilon}$ is $\frac{1}{\sqrt{\varepsilon}}$ of the original velocity $V$. Similarly, $P(X, Y)=P^{\varepsilon}(x, y)$. In these new variables, the Navier-Stokes equations (1.1) now read

$$
\begin{aligned}
U^{\varepsilon} U_{x}^{\varepsilon}+V^{\varepsilon} U_{y}^{\varepsilon}+P_{x}^{\varepsilon} & =U_{y y}^{\varepsilon}+\varepsilon U_{x x}^{\varepsilon} \\
U^{\varepsilon} V_{x}^{\varepsilon}+V^{\varepsilon} V_{y}^{\varepsilon}+\frac{P_{y}^{\varepsilon}}{\varepsilon} & =V_{y y}^{\varepsilon}+\varepsilon V_{x x}^{\varepsilon} \\
U_{x}^{\varepsilon}+V_{y}^{\varepsilon} & =0 .
\end{aligned}
$$


Throughout the paper, we perform our analysis directly on these scaled equations together with the same no-slip boundary conditions (1.2). Prandtl then hypothesized that the Navier-Stokes flow can be approximately decomposed into two parts:

$$
\begin{aligned}
{\left[U^{\varepsilon}, V^{\varepsilon}\right] } & \approx\left[u_{e}^{0}(\sqrt{\varepsilon} y), 0\right]+[\bar{u}(x, y), \bar{v}(x, y)] \\
P^{\varepsilon} & \approx \bar{p}(x, y)
\end{aligned}
$$

in which $u_{e}^{0}(\sqrt{\varepsilon} y)$ denotes the Euler flow as in (1.3). Putting the ansatz into the Navier-Stokes equations (1.4) and collecting the leading terms in $\varepsilon$, we obtain the Prandtl layer corrector $[\bar{u}, \bar{v}]$ which satisfies

$$
\begin{aligned}
{\left[u_{e}+\bar{u}\right] \bar{u}_{x}+v \bar{u}_{y}+\bar{p}_{x} } & =\bar{u}_{y y} \\
\bar{u}_{x}+\bar{v}_{y} & =0
\end{aligned}
$$

where $\bar{p}_{x}=\bar{p}_{x}(x)$, since by the second equation in (1.4), $\bar{p}_{y}=0$. Evaluating the first equation at $y=\infty$, one gets $\bar{p}_{x}=0$, which is precisely due to the assumption that the trace of Euler on the boundary does not depend on $x$ (a.k.a, Bernoulli's law). Hence, the boundary layer satisfies

$$
\bar{u}(x, 0)=-u_{e}+u_{b}<0, \quad \bar{v}(x, 0)=0, \quad \lim _{y \rightarrow \infty} \bar{u}(x, y)=0 .
$$

The Prandtl layer $[\bar{u}, \bar{v}]$ is subject to an "initial" condition at $x=0$ :

$$
\bar{u}(0, y)=\bar{u}_{0}(y)
$$

Regarded as one of the most important achievements of modern fluid mechanics, Prandtl's the boundary layer expansion (1.5) connects the theory of ideal fluid (Euler flows) with the real fluid (Navier-Stokes flows) near the boundary, for a large Reynolds number (or equivalently, $\varepsilon \ll 1$ ). Such a theory has led to tremendous applications and advances in science and engineering. In particular, since the Prandtl layer solution $[\bar{u}, \bar{v}]$ satisfies an evolution equation in $x$, it is much easier to compute its solutions numerically than those of the original NS flows $\left[U^{\varepsilon}, V^{\varepsilon}\right]$ which satisfy an elliptic boundary-value problem. Many other shear layer phenomena in fluids, such as wake flows ([17, page 187]), plane jet flows ([17, page 190]), as well as shear layers between two parallel flows, can also be described by the Prandtl layer theory (1.6) and (1.7).

In spite of the huge success of Prandtl's boundary layer theory in applications, it remains an outstanding open problem to rigorously justify the validity of expansion (1.5) in the inviscid limit. The purpose of this paper is to provide an affirmative answer along this direction.

As it turns out, we will need higher order approximations, as compared to (1.5), in order to be able to control the remainders. Precisely, we search for asymptotic expansions of the scaled Navier-Stokes solutions $\left[U^{\varepsilon}, V^{\varepsilon}, P^{\varepsilon}\right]$ in the following form:

$$
\begin{aligned}
& U^{\varepsilon}(x, y)=u_{e}^{0}(\sqrt{\varepsilon} y)+u_{p}^{0}(x, y)+\sqrt{\varepsilon} u_{e}^{1}(x, \sqrt{\varepsilon} y)+\sqrt{\varepsilon} u_{p}^{1}(x, y)+\varepsilon^{\gamma+\frac{1}{2}} u^{\varepsilon}(x, y) \\
& V^{\varepsilon}(x, y)=v_{p}^{0}(x, y)+v_{e}^{1}(x, \sqrt{\varepsilon} y)+\sqrt{\varepsilon} v_{p}^{1}(x, y)+\varepsilon^{\gamma+\frac{1}{2}} v^{\varepsilon}(x, y) \\
& P^{\varepsilon}(x, y)=\sqrt{\varepsilon} p_{e}^{1}(x, \sqrt{\varepsilon} y)+\sqrt{\varepsilon} p_{p}^{1}(x, y)+\varepsilon p_{p}^{2}(x, y)+\varepsilon^{\gamma+\frac{1}{2}} p^{\varepsilon}(x, y)
\end{aligned}
$$

for some $\gamma>0$, in which $\left[u_{e}^{j}, v_{e}^{j}, p_{e}^{j}\right]$ and $\left[u_{p}^{j}, v_{p}^{j}, p_{p}^{j}\right]$, with $j=0,1$, denote the Euler and Prandtl profiles, respectively, and $\left[u^{\varepsilon}, v^{\varepsilon}, p^{\varepsilon}\right]$ collects all the remainder solutions. Here, we note that these 
profile solutions also depend on $\varepsilon$, and the Euler flows are always evaluated at $(x, \sqrt{\varepsilon} y)$, whereas the Prandtl profiles are at $(x, y)$.

Formally speaking, plugging the above ansatz into (1.4) and matching the order in $\varepsilon$, we easily get that $\left[u_{p}^{0}, v_{p}^{0}, 0\right]$ solves the nonlinear Prandtl equation (1.6), whereas the next Euler profile $\left[u_{e}^{1}, v_{e}^{1}, p_{e}^{1}\right]$ solves the linearized Euler equations around $\left(u_{e}^{0}, 0\right)$ :

$$
\begin{array}{r}
u_{e}^{0} u_{e x}^{1}+v_{e}^{1} u_{e y}^{0}+p_{e x}^{1}=0 \\
u_{e}^{0} v_{e x}^{1}+p_{e y}^{1}=0 \\
u_{e x}^{1}+v_{e y}^{1}=0
\end{array}
$$

or equivalently in the vorticity formulation,

$$
-u_{e}^{0} \Delta v_{e}^{1}+u_{e y y}^{0} v_{e}^{1}=0,
$$

with $\left[u_{e}^{1}, p_{e}^{1}\right]$ being recovered from the last two equations in (1.10). The next Prandtl layer $\left[u_{p}^{1}, v_{p}^{1}, p_{p}^{1}\right]$ solves the linearized Prandtl equations around $\left[u_{e}+u_{p}^{0}, v_{p}^{0}+v_{e}^{1}\right]$, with a source term $E_{p}$ :

$$
\begin{aligned}
\left(u_{e}+u_{p}^{0}\right) u_{p x}^{1}+u_{p}^{1} u_{p x}^{0}+\left(v_{p}^{0}+v_{e}^{1}\right) u_{p y}^{1}+v_{p}^{1} u_{p x}^{0}+p_{p x}^{1}-u_{p y y}^{1} & =E_{p}, \\
u_{p x}^{1}+v_{p y}^{1} & =0,
\end{aligned}
$$

with $p_{p}^{1}=p_{p}^{1}(x)$. Certainly, the remainder solutions $\left[u^{\varepsilon}, v^{\varepsilon}, p^{\varepsilon}\right]$ solve the linearized Navier-Stokes equations around the approximate solutions, with a source that contains nonlinearity in $\left[u^{\varepsilon}, v^{\varepsilon}\right]$; see Section 5 for details.

Note however that as we deal with functions in Sobolev spaces, all profile solutions are required to vanish at $y=\infty$. As it will be clear in the text, the actual Prandtl layers will introduce nonzero normal velocity at infinity, and is one of the issues in controlling the remainders, since the profiles then won't even be integrable. As a result, our Prandtl layers $\left[u_{p}^{1}, v_{p}^{1}\right]$ in the expansion (1.9) are being cut-off for large $y$ of the actual layers solving (1.12). In Section 2, we shall provide detailed construction of the approximate solutions and derive sufficient estimates for our analysis.

\subsection{Boundary conditions}

The zeroth Euler flow $u_{e}^{0}$ is given. Due to the no-slip boundary condition at $y=0$, we require that

$$
u_{e}+u_{p}^{0}(x, 0)=u_{b}, \quad v_{e}^{1}(x, 0)+v_{p}^{0}(x, 0)=0,
$$

from the zeroth order in $\varepsilon$ of the expansion (1.9), and

$$
u_{p}^{1}(x, 0)=-u_{e}^{1}(x, 0), \quad v_{p}^{1}(x, 0)=0,
$$

from the $\sqrt{\varepsilon}$-order layers. We also assume that

$$
\lim _{y \rightarrow \infty} u_{p}^{0}(x, y)=0, \quad \lim _{y \rightarrow \infty} u_{p}^{1}(x, y)=0, \quad \lim _{Y \rightarrow \infty}\left[u_{e}^{j}, v_{e}^{j}\right](x, Y)=0 .
$$

The normal velocities $v_{p}^{0}(x, y), v_{p}^{1}(x, y)$ in the boundary layers are constructed from $u_{p}^{0}(x, y), u_{p}^{1}(x, y)$, respectively, through the divergence-free condition. We note that in general $\lim _{y \rightarrow \infty} v_{p}^{j}(x, y) \neq 0$ and hence, cut-off functions will be introduced to localize $v_{p}^{j}$. 
Next, we discuss boundary conditions at $x=0, L$. Since the Prandtl layers solve parabolic-type equations, we require only "initial" conditions at $x=0$ :

$$
u_{p}^{0}(0, y)=\bar{u}_{0}(y), \quad u_{p}^{1}(0, y)=\bar{u}_{1}(y)
$$

whereas we prescribe boundary values for the Euler profiles at both $x=0, L$ :

$$
u_{e}^{1}(0, Y)=u_{b}^{1}(Y), \quad v_{e}^{1}(0, Y)=V_{b 0}(Y), \quad v_{e}^{1}(L, Y)=V_{b L}(Y)
$$

with compatibility conditions $V_{b 0}(0)=v_{p}^{0}(0,0), V_{b L}(0)=v_{p}^{0}(L, 0)$ at the corners of the domain $[0, L] \times \mathbb{R}_{+}$.

Finally, we impose the following boundary conditions for the remainder solution $\left[u^{\varepsilon}, v^{\varepsilon}\right]$ :

$$
\begin{array}{rlrl}
{\left[u^{\varepsilon}, v^{\varepsilon}\right]_{y=0}} & =0 \text { (no-slip), } \quad\left[u^{\varepsilon}, v^{\varepsilon}\right]_{x=0}=0 \quad \text { (Dirichlet), } \\
p^{\varepsilon}-2 \varepsilon u_{x}^{\varepsilon} & =0, \quad u_{y}^{\varepsilon}+\varepsilon v_{x}^{\varepsilon}=0 & \text { at } x=L \text { (Neumann or stress-free). }
\end{array}
$$

Certainly, one may wish to consider different boundary conditions for $\left[u^{\varepsilon}, v^{\varepsilon}\right]$ at $x=L$. However, to avoid a possible formation of boundary layers with respect to $x$ near the boundary $x=L$, the above Neumann stress-free condition appears the most convenient candidate to impose.

\subsection{Main result and discussions}

We are ready to state our main result:

Theorem 1.1. Let $u_{e}^{0}(Y)$ be a given smooth Euler flow, and let $u_{b}^{1}(Y), V_{b 0}(Y), V_{b L}(Y)$, with $Y=$ $\sqrt{\varepsilon} y$, and $\bar{u}_{0}(y)$ and $\bar{u}_{1}(y)$ be given smooth data and decay exponentially fast at infinity in their arguments, and let $u_{b}$ be a positive constant. We assume that $\left|V_{b L}(Y)-V_{b 0}(Y)\right| \lesssim L$ for small $L$, and

$$
\min _{0 \leq y \leq \infty}\left\{u_{e}^{0}(\sqrt{\varepsilon} y)+\bar{u}_{0}(y)\right\}>0 .
$$

Then, there exists a positive number $L$ that depends only on the given data so that the boundary layer expansions (1.9), with the profiles satisfying the boundary conditions in Section 1.1, hold for $\gamma \in\left(0, \frac{1}{4}\right)$. Precisely, $\left[U^{\varepsilon}, V^{\varepsilon}, P^{\varepsilon}\right]$ as defined in (1.9) is the unique solution to the Navier-Stokes equations (1.4), so that the remainder solutions $\left[u^{\varepsilon}, v^{\varepsilon}\right]$ satisfy

$$
\left\|\nabla_{\varepsilon} u^{\varepsilon}\right\|_{L^{2}}+\varepsilon^{\frac{1}{2}}\left\|\nabla_{\varepsilon} v^{\varepsilon}\right\|_{L^{2}}+\varepsilon^{\frac{\gamma}{2}}\left\|u^{\varepsilon}\right\|_{L^{\infty}}+\varepsilon^{\frac{1}{2}+\frac{\gamma}{2}}\left\|v^{\varepsilon}\right\|_{L^{\infty}} \leq C_{0}
$$

for some constant $C_{0}$ that depends only on the given data. Here, $\nabla_{\varepsilon}=\left(\sqrt{\varepsilon} \partial_{x}, \partial_{y}\right)$, and $\|\cdot\|_{L^{p}}$ denotes the usual $L^{p}$ norm over $[0, L] \times \mathbb{R}_{+}$.

As a direct corollary of our main theorem above, we obtain the inviscid limit of the steady Navier-Stokes flows, with prescribed data up to the order of square root of viscosity.

Corollary 1.2. Under the same assumption as made in Theorem 1.1, there are exact solutions $[U, V]$ to the original Navier-Stokes equations (1.1) on $\Omega=[0, L] \times \mathbb{R}_{+}$, with $L$ being as in Theorem 1.1, so that

$$
\begin{aligned}
\sup _{(x, y) \in \Omega}\left|U(x, y)-u_{e}^{0}(y)-u_{p}^{0}\left(x, \frac{y}{\sqrt{\varepsilon}}\right)\right| & \lesssim \varepsilon^{\frac{1}{2}} \\
\sup _{(x, y) \in \Omega}\left|V(x, y)-\sqrt{\varepsilon} v_{p}^{0}\left(x, \frac{y}{\sqrt{\varepsilon}}\right)-\sqrt{\varepsilon} v_{e}^{1}(x, y)\right| & \lesssim \varepsilon^{\frac{\gamma}{2}+\frac{1}{2}}
\end{aligned}
$$


as $\varepsilon \rightarrow 0$, for given Euler flow $u_{e}^{0}$, the constructed Euler flows $\left[u_{e}^{1}, v_{e}^{1}\right]$ and Prandtl layers $\left[u_{p}^{0}, \sqrt{\varepsilon} v_{p}^{0}\right]$. In particular, we have the convergence $(U, V) \rightarrow\left(u_{e}^{0}, 0\right)$ in the usual $L^{p}$ norm, with a rate of convergence of order $\varepsilon^{1 / 2 p}, 1 \leq p<\infty$, in the inviscid limit of $\varepsilon \rightarrow 0$.

Remark 1.3. We note that the Prandtl layers $\left[u_{p}^{0}, v_{p}^{0}\right]$ are not exactly the layers $[\bar{u}, \bar{v}]$ solving (1.6)-(1.8). Indeed, whereas $u_{p}^{0}=\bar{u}$, we have the normal velocity $v_{p}^{0}=\int_{y}^{\infty} \bar{u}_{x}$, but $\bar{v}=-\int_{0}^{y} \bar{u}_{x}$ for the true Prandtl layer. The introduction of the Euler layer $\left[u_{e}^{1}, v_{e}^{1}\right]$ was necessary to correct this.

Let us give a few comments about the main result. First, the nonzero condition (1.14) and $u_{b}>0$ are naturally related to the situation where boundary layers are near a moving plate: such as a wake flow of a moving body, a moving plane jet flow, and a shear layer between two parallel flows. It may also be related to the well-known fact in engineering that injection of moving fluids at the surface prevents the boundary layer separation.

It is widely known that the mathematical study of Prandtl boundary layers and the inviscid limit problem is challenging due to its characteristic nature at the boundary (that is, $v=0$ at $y=0)$ and the instability of generic boundary layers $([6,77,8,9])$. Here, for steady flows, we are able to justify the Prandtl boundary layer theory. There are several issues to overcome. The first is to carefully construct Euler and Prandtl solutions and derive sufficient estimates. The complication occurs due to the fact that we have to truncate the actual layers in order to fit in our functional framework, and the lack of a priori estimates for linearized Prandtl equations. The construction of the approximate solutions is done in Section 2.

Next, once the approximate solutions are constructed, we need to derive stability estimates for the remainder solutions. Due to the limited regularity obtained for the Prandtl layers $\left[u_{p}^{1}, v_{p}^{1}\right]$, we shall study the linearization around the following approximate solutions:

$$
u_{s}(x, y):=u_{e}^{0}(\sqrt{\varepsilon} y)+u_{p}^{0}(x, y)+\sqrt{\varepsilon} u_{e}^{1}(x, \sqrt{\varepsilon} y), \quad v_{s}(x, y):=v_{p}^{0}(x, y)+v_{e}^{1}(x, \sqrt{\varepsilon}) .
$$

A straightforward calculation (Section 5) yields the equations for the remainder solutions $\left[u^{\varepsilon}, v^{\varepsilon}, p^{\varepsilon}\right]$ in (1.9):

$$
\begin{aligned}
u_{s} u_{x}^{\varepsilon}+u^{\varepsilon} u_{s x}+v_{s} u_{y}^{\varepsilon}+v^{\varepsilon} u_{s y}+p_{x}^{\varepsilon}-\Delta_{\varepsilon} u^{\varepsilon} & =R_{1}\left(u^{\varepsilon}, v^{\varepsilon}\right) \\
u_{s} v_{x}^{\varepsilon}+u^{\varepsilon} v_{s x}+v_{s} v_{y}^{\varepsilon}+v^{\varepsilon} v_{s y}+\frac{p_{y}^{\varepsilon}}{\varepsilon}-\Delta_{\varepsilon} v^{\varepsilon} & =R_{2}\left(u^{\varepsilon}, v^{\varepsilon}\right) \\
u_{x}^{\varepsilon}+v_{y}^{\varepsilon} & =0
\end{aligned}
$$

with $\Delta_{\varepsilon}=\partial_{y}^{2}+\varepsilon \partial_{x}^{2}$. Here, $\left[u_{s}, v_{s}\right]$ denotes the leading approximate solutions (see (1.15) ), and the remainders $R_{1,2}\left(u^{\varepsilon}, v^{\varepsilon}\right)$ are defined as in (5.4). The standard energy estimate (Section 3.1) yields precisely a control on $\left\|\nabla_{\varepsilon} u^{\varepsilon}\right\|_{L^{2}}$ and $\sqrt{\varepsilon}\left\|\nabla_{\varepsilon} v^{\varepsilon}\right\|_{L^{2}}$, but cannot close the analysis, due to the large convective term: $\int u_{s y} u^{\varepsilon} v^{\varepsilon}$, for instance. Indeed, this is a very common and central difficulty in the stability theory of boundary layers.

The most crucial ingredient (Section [3.2) in the proof is to give bound on $\left\|\nabla_{\varepsilon} v^{\varepsilon}\right\|_{L^{2}}$ (in order one, instead of order $\sqrt{\varepsilon}$ from the energy estimate). The key is to study the vorticity equation,

$$
-u_{s} \Delta_{\epsilon} v^{\varepsilon}+v_{s} \Delta_{\epsilon} u^{\varepsilon}-u^{\varepsilon} \Delta_{\epsilon} v_{s}+v^{\varepsilon} \Delta_{\epsilon} u_{s}=\Delta_{\epsilon} \omega^{\varepsilon}+R_{1 y}-\varepsilon R_{2 x} .
$$

with a new multiplier $\frac{v^{\varepsilon}}{u_{s}}$. Here, the assumption (1.14) and $u_{b}>0$, together with the Maximum Principle for the Prandtl equations (see estimate (2.8)), assure that $u_{s}$ is bounded away from zero. Formally, without worrying about boundary terms, the integral $\int \Delta_{\varepsilon} \omega^{\varepsilon} v^{\varepsilon}$ vanishes. Hence, the 
leading term in the vorticity estimate lies in the convection: $-u_{s} \Delta_{\epsilon} v^{\varepsilon}+v^{\varepsilon} \Delta_{\epsilon} u_{s}$, or to leading order in the boundary layer analysis, $-u_{s} \partial_{y}^{2} v^{\varepsilon}+u_{s y y} v^{\varepsilon}$. Our key observation is then the positivity of the second-order operator:

$$
-\partial_{y y}+\frac{u_{s y y}}{u_{s}}
$$

Indeed, a direct calculation yields

$$
\begin{aligned}
-\int v_{y y} v & =\int\left|v_{y}\right|^{2}=\int\left|\partial_{y}\left\{\frac{v}{u_{s}} u_{s}\right\}\right|^{2} \\
& =\int u_{s}^{2}\left|\partial_{y}\left\{\frac{v}{u_{s}}\right\}\right|^{2}+\int u_{s y}^{2}\left\{\frac{v}{u_{s}}\right\}^{2}+2 \int \partial_{y}\left\{\frac{v}{u_{s}}\right\} u_{s} u_{s y}\left\{\frac{v}{u_{s}}\right\} \\
& =\int u_{s}^{2}\left|\partial_{y}\left\{\frac{v}{u_{s}}\right\}\right|^{2}+\int u_{s y}^{2}\left\{\frac{v}{u_{s}}\right\}^{2}-\int\left\{\frac{v}{u_{s}}\right\}^{2}\left[u_{s} u_{s y}\right]_{y} \\
& =\int u_{s}^{2}\left|\partial_{y}\left\{\frac{v}{u_{s}}\right\}\right|^{2}-\int \frac{u_{s y y}}{u_{s}} v^{2}
\end{aligned}
$$

which gives the positivity estimate:

$$
\int\left\{-\partial_{y y}+\frac{u_{s y y}}{u_{s}}\right\} v v=\int\left|\partial_{y} v\right|^{2}+\int \frac{u_{s y y}}{u_{s}} v^{2}=\int u_{s}^{2}\left|\partial_{y}\left\{\frac{v}{u_{s}}\right\}\right|^{2}>0 .
$$

The desired bound on $v_{y}^{\varepsilon}$, and in fact, $\nabla_{\varepsilon} v^{\varepsilon}$ is derived from this positivity estimate and the weighted estimates from the vorticity equation. Precisely,

$$
\begin{aligned}
\iint v_{y}^{2} & =\iint\left\{\partial_{y}\left[u_{s}\left\{\frac{v}{u_{s}}\right\}\right]\right\}^{2} \leq 2 \iint u_{s}^{2}\left\{\frac{v}{u_{s}}\right\}_{y}^{2}+2 \iint\left\{\partial_{y} u_{s}\right\}^{2}\left\{\frac{v}{u_{s}}\right\}^{2} \\
& \leq 2 \iint u_{s}^{2}\left|\partial_{y}\left\{\frac{v}{u_{s}}\right\}\right|^{2}+2 \iint\left[\partial_{y}\left\{\frac{v}{u_{s}}\right\}\right]^{2} \times \sup _{x} \int y\left\{\partial_{y} u_{s}\right\}^{2} d y \\
& \leq 2 \iint u_{s}^{2}\left|\partial_{y}\left\{\frac{v}{u_{s}}\right\}\right|^{2}\left[1+\frac{1}{\min u_{s}^{2}} \times \sup _{x} \int y\left\{\partial_{y} u_{s}\right\}^{2} d y\right] \\
& \lesssim \iint u_{s}^{2}\left|\partial_{y}\left\{\frac{v}{u_{s}}\right\}\right|^{2},
\end{aligned}
$$

in which the last inequality used the estimate (3.8) on $u_{s}$.

In addition, the Dirichlet boundary condition at $x=0$ and the stress-free boundary condition at $x=L$ as imposed in (1.13) are carefully designed to ensure boundary contributions at $x=0$ and $x=L$ are controllable.

Our second ingredient is to derive $L^{\infty}$ estimate for the remainder solution $\left[u^{\varepsilon}, v^{\varepsilon}\right]$ and to close the nonlinear analysis; Sections 4 and 5. We have to overcome the issue of regularity of solutions to the elliptic problem in domains with corners. In particular, it is a subtlety to justify the integrability of all terms in integration by parts, given the limited regularity provided for the solution near the corners. We remark that in the case $u_{b}=0$, our analysis does not directly apply due to the presence of zero points of the profile solutions $u_{s}$, and hence the function $\frac{v^{\varepsilon}}{u_{s}}$ can no longer be used as a multiplier. Our positivity estimate is lost in this limiting, but classical, case. 
Finally, the third ingredient is the construction of profiles (or approximate solutions) which enables us to establish the error estimates and to close our nonlinear iteration. Such constructions are delicate (Section 2), due to the regularity requirement of $v_{p x x}^{1}$ in the remainder $R_{2}\left(u^{\varepsilon}, v^{\varepsilon}\right.$ ). In order to control it, we need to create artificial new boundary layer at $y=0$ in (2.30) to guarantee sufficient regularity for the first order Euler correction $\left[u_{e}^{1}, v_{e}^{1}, p_{e}^{1}\right]$. More importantly, to construct both $v_{e}^{1}$ and $v_{p}^{1}$, the positivity estimate (1.16) once again plays the decisive role; see Sections 2.2 and 2.4.

We are not aware of any work in the literature that deals with the validity of the Prandtl boundary layer theory for the steady Navier-Stokes flows. For unsteady flows, there are very interesting contributions [1, 15, 16] in the analyticity framework, 10] in the case where the initial vorticity is assumed to be away from the boundary, or [11 for special Navier-Stokes flows. An analogous program for unsteady flows as done for the steady case in the precent paper appears not possible, due to the fact that (unsteady) boundary layers are known to be very unstable; see, for instance, [5, 6, 7, 8, 9].

Notation. Throughout the paper, we shall use $\langle y\rangle=\sqrt{1+y^{2}}$, and $\|\cdot\|_{L^{p}}$ or occasionally $\|\cdot\|_{p}$ to denote the usual $L^{p}$ norms, $p \geq 1$, with integration taken over $\Omega=[0, L] \times \mathbb{R}_{+}$. We also use $\|\cdot\|_{L^{p}(0, L)}$ and $\|\cdot\|_{L^{p}\left(\mathbb{R}_{+}\right)}$to denote the $L^{p}$ norms with integration taken over $[0, L]$ and $\mathbb{R}_{+}$, respectively. We shall denote by $C\left(u_{s}, v_{s}\right)$ a universal constant that depends only on the given Euler flow $u_{e}^{0}$ and boundary data. Occasionally, we simply write $C$ or use the notation $\lesssim$ in the estimates. By uniform estimates, we always mean those that are independent of smallness of $\varepsilon$ and $L$. The smallness of $L$ is determined depending only on the given data, whereas $\varepsilon$ is taken arbitrarily small, once the given data and $L$ are fixed. In particular, $\varepsilon \ll L$.

\section{Construction of the approximate solutions}

In order to construct the approximate solutions, we plug the Ansatz (1.9) into the scaled NavierStokes equations (1.4), and match the order in $\varepsilon$ to determine the equations for the profiles. For our own convenience, let us introduce

$$
\begin{aligned}
& u_{\text {app }}(x, y)=u_{e}^{0}(\sqrt{\varepsilon} y)+u_{p}^{0}(x, y)+\sqrt{\varepsilon} u_{e}^{1}(x, \sqrt{\varepsilon} y)+\sqrt{\varepsilon} u_{p}^{1}(x, y) \\
& v_{\text {app }}(x, y)=v_{p}^{0}(x, y)+v_{e}^{1}(x, \sqrt{\varepsilon} y)+\sqrt{\varepsilon} v_{p}^{1}(x, y) \\
& p_{\text {app }}(x, y)=\sqrt{\varepsilon} p_{e}^{1}(x, \sqrt{\varepsilon} y)+\sqrt{\varepsilon} p_{p}^{1}(x, y)+\varepsilon p_{p}^{2}(x, y) .
\end{aligned}
$$

We then calculate the error caused by the approximation:

$$
\begin{aligned}
& R_{\mathrm{app}}^{u}:=\left[u_{\mathrm{app}} \partial_{x}+v_{\mathrm{app}} \partial_{y}\right] u_{\mathrm{app}}+\partial_{x} p_{\mathrm{app}}-\Delta_{\varepsilon} u_{\mathrm{app}} \\
& R_{\mathrm{app}}^{v}:=\left[u_{\mathrm{app}} \partial_{x}+v_{\mathrm{app}} \partial_{y}\right] v_{\mathrm{app}}+\frac{1}{\varepsilon} \partial_{y} p_{\mathrm{app}}-\Delta_{\varepsilon} v_{\mathrm{app}}
\end{aligned}
$$

or explicitly,

$$
\begin{aligned}
R_{\mathrm{app}}^{u}= & {\left[\left\{u_{e}^{0}+u_{p}^{0}+\sqrt{\varepsilon}\left[u_{e}^{1}+u_{p}^{1}\right]\right\} \partial_{x}+\left\{v_{p}^{0}+v_{e}^{1}+\sqrt{\varepsilon} v_{p}^{1}\right\} \partial_{y}\right]\left\{u_{e}^{0}+u_{p}^{0}+\sqrt{\varepsilon}\left[u_{e}^{1}+u_{p}^{1}\right]\right\} } \\
& +\sqrt{\varepsilon} \partial_{x}\left\{p_{e}^{1}+p_{p}^{1}+\sqrt{\varepsilon} p_{p}^{2}\right\}-\left(\partial_{y}^{2}+\varepsilon \partial_{x}^{2}\right)\left\{u_{e}^{0}+u_{p}^{0}+\sqrt{\varepsilon}\left[u_{e}^{1}+u_{p}^{1}\right]\right\} \\
R_{\mathrm{app}}^{v}= & {\left[\left\{u_{e}^{0}+u_{p}^{0}+\sqrt{\varepsilon}\left[u_{e}^{1}+u_{p}^{1}\right]\right\} \partial_{x}+\left\{v_{p}^{0}+v_{e}^{1}+\sqrt{\varepsilon} v_{p}^{1}\right\} \partial_{y}\right]\left\{v_{p}^{0}+v_{e}^{1}+\sqrt{\varepsilon} v_{p}^{1}\right\} } \\
& +\frac{1}{\sqrt{\varepsilon}} \partial_{y}\left\{p_{e}^{1}+p_{p}^{1}+\sqrt{\varepsilon} p_{p}^{2}\right\}-\left(\partial_{y}^{2}+\varepsilon \partial_{x}^{2}\right)\left\{v_{p}^{0}+v_{e}^{1}+\sqrt{\varepsilon} v_{p}^{1}\right\},
\end{aligned}
$$


in which we recall that the Euler profiles are always evaluated at $(x, z)=(x, \sqrt{\varepsilon} y)$. We shall construct the approximate solutions so that $R_{\text {app }}^{u, v}$ are being small in $\varepsilon$. In this long section, we shall prove that

Proposition 2.1. Under the same assumptions as in Theorem 1.1, there are approximate solutions $\left[u_{\mathrm{app}}, v_{\mathrm{app}}, p_{\mathrm{app}}\right]$ so that

$$
\left\|R_{\text {app }}^{u}\right\|_{L^{2}}+\sqrt{\varepsilon}\left\|R_{\text {app }}^{v}\right\|_{L^{2}} \leq C(L, \kappa) \varepsilon^{3 / 4-\kappa},
$$

for arbitrarily small $\kappa>0$. Furthermore, there hold various regularity estimates on the approximate solutions which are summarized in Corollary 2.3 for the zeroth-order Prandtl layers $\left[u_{p}^{0}, v_{p}^{0}\right]$, Section 2.3.1 for the Euler profiles $\left[u_{e}^{1}, v_{e}^{1}\right]$, and Section 2.4.2 for the Prandtl layers $\left[u_{p}^{1}, v_{p}^{1}\right]$.

\subsection{Zeroth-order Prandtl layers}

The (leading) zeroth order terms on the right-hand side of (2.2a) consist of

$$
R^{u, 0}:=\left\{u_{e}^{0}+u_{p}^{0}\right\}\left\{u_{e}^{0}+u_{p}^{0}\right\}_{x}+\left\{v_{p}^{0}+v_{e}^{1}\right\}\left\{u_{e}^{0}+u_{p}^{0}\right\}_{y}-\left\{u_{e}^{0}+u_{p}^{0}\right\}_{y y}
$$

in which we note that $\left\{u_{e}^{0}\right\}_{x}=0$. Since the Euler flows are evaluated at $(x, z)=(x, \sqrt{\varepsilon} y)$, we may write

$$
\left\{v_{p}^{0}+v_{e}^{1}\right\} \partial_{y} u_{e}^{0}=\sqrt{\varepsilon}\left\{v_{p}^{0}+v_{e}^{1}\right\} u_{e z}^{0}
$$

and, with $u_{e}=u_{e}^{0}(0)$,

$$
u_{e}^{0} u_{p x}^{0}+v_{e}^{1} u_{p y}^{0}=u_{e} u_{p x}^{0}+v_{e}^{1}(x, 0) u_{p y}^{0}+\sqrt{\varepsilon} u_{e z}(\sqrt{\varepsilon} y) y u_{p x}^{0}+\sqrt{\varepsilon} v_{e z}^{1}(\sqrt{\varepsilon} y) y u_{p y}^{0}+E^{0}
$$

in which $E^{0}$ satisfies

$$
\begin{aligned}
E^{0} & :=\sqrt{\varepsilon} u_{p x}^{0} \int_{0}^{y}\left\{u_{e z}^{0}(\sqrt{\varepsilon} \theta)-u_{e z}^{0}(\sqrt{\varepsilon} y)\right\} d \theta+\sqrt{\varepsilon} u_{p y}^{0} \int_{0}^{y}\left\{v_{e z}^{1}(\sqrt{\varepsilon} \theta)-v_{e z}^{1}(\sqrt{\varepsilon} y)\right\} d \theta \\
& =\varepsilon u_{p x}^{0} \int_{0}^{y} \int_{y}^{\theta} u_{e z z}^{0}(\sqrt{\varepsilon} \tau) d \tau d \theta+\varepsilon u_{p y}^{0} \int_{0}^{y} \int_{y}^{\theta} v_{e z z}^{1}(\sqrt{\varepsilon} \tau) d \tau d \theta .
\end{aligned}
$$

In particular, $E_{0}$ is in the high order in $\varepsilon$, as to be proved rigorously in the next section; see (2.40). To leading order, this yields the nonlinear Prandtl problem for $u_{p}^{0}$ :

$$
\left\{\begin{array}{c}
\left\{u_{e}+u_{p}^{0}\right\} u_{p x}^{0}+\left\{v_{p}^{0}+v_{e}^{1}(x, 0)\right\} u_{p y}^{0}=u_{p y y}^{0}, \quad v_{p}^{0}(x, y):=\int_{y}^{\infty} u_{p x}^{0} d y \\
u_{p}^{0}(x, 0)=u_{b}-u_{e}, \quad v_{p}^{0}(x, 0)+v_{e}^{1}(x, 0)=0, \quad u_{p}^{0}(0, y)=\bar{u}_{0}(y)
\end{array}\right.
$$

Having constructed the Prandtl layer $\left[u_{p}^{0}, v_{p}^{0}\right]$, the zeroth order term $R^{u, 0}$ is reduced to

$$
R^{u, 0}=\sqrt{\varepsilon}\left\{v_{p}^{0}+v_{e}^{1}\right\} u_{e z}^{0}+\sqrt{\varepsilon} u_{e z}(\sqrt{\varepsilon} y) y u_{p x}^{0}+\sqrt{\varepsilon} v_{e z}^{1}(\sqrt{\varepsilon} y) y u_{p y}^{0}-\varepsilon u_{e z z}^{0}+E^{0},
$$

which will be put into the next order in $\varepsilon$.

Lemma 2.2. Let $u_{p}^{0}(0, y):=\bar{u}_{0}(y)$ be an arbitrary smooth boundary data for the Prandtl layer at $x=0$. Assume that $\min _{y}\left\{u_{e}+\bar{u}_{0}(y)\right\}>0$. Then, there exists a positive number $L$ so that the 
problem (2.4) has the unique smooth solution $u_{p}^{0}(x, y)$ in $[0, L] \times \mathbb{R}_{+}$. Furthermore, for all $n \geq 0$, $k \geq 0$, there exists a constant $C_{0}\left(n, k, \bar{u}_{0}\right)$ so that there holds the uniform bound:

$$
\sup _{x \in[0, L]}\left\|\langle y\rangle^{n / 2} \partial_{x}^{k} u_{p}^{0}\right\|_{L^{2}\left(\mathbb{R}_{+}\right)}+\left\|\langle y\rangle^{n / 2} \partial_{x}^{k} \partial_{y} u_{p}^{0}\right\|_{L^{2}\left(0, L ; L^{2}\left(\mathbb{R}_{+}\right)\right)} \leq C_{0}\left(n, k, \bar{u}_{0}\right),
$$

with $\langle y\rangle=\sqrt{1+|y|^{2}}$. Here, the constant $C\left(n, k, \bar{u}_{0}\right)$ depends on $n, k$, and the $\langle y\rangle^{n}$-weighted $H^{2 k}\left(\mathbb{R}_{+}\right)$norm of the boundary value $\bar{u}_{0}(y)$.

Corollary 2.3. Let $u_{p}^{0}$ be the Prandtl layer constructed as in Lemma 2.2. Then, there holds

$$
\sup _{x \in[0, L]}\left\|\langle y\rangle^{n / 2} \partial_{x}^{k} \partial_{y}^{j}\left[u_{p}^{0}, v_{p}^{0}\right]\right\|_{L^{2}\left(\mathbb{R}_{+}\right)} \leq C_{0}\left(n, k, j, \bar{u}_{0}\right),
$$

for arbitrary $n, k, j$.

Proof. Indeed, the proof follows directly from Lemma 2.2 and a use of equations (2.4) for the Prandtl layer to bound $\partial_{y}^{2} u_{p}^{0}$ by those of lower-order derivative terms.

Proof of Lemma 2.2. Let $u_{e}=u_{e}^{0}(0)$. Following Oleinik [12], we use the von Mises transformation:

$$
\eta:=\int_{0}^{y}\left(u_{e}+u_{p}^{0}(x, \theta)\right) d \theta, \quad \mathbf{w}(x, \eta):=u_{e}+u_{p}^{0}(x, y(\eta)) .
$$

The function $\mathbf{w}$ then solves

$$
\mathbf{w}_{x}=\left\{\mathbf{w}_{\eta}\right\}_{\eta}
$$

on $[0, L] \times \mathbb{R}_{+}$. Note that by the standard Maximum Principle (to the equation for $\mathbf{w}^{2}$ ), we have

$$
\mathbf{w} \geq \min _{y}\left\{u_{b}, u_{e}+\left.u_{p}^{0}\right|_{x=0}\right\} \geq c_{0}>0
$$

for some positive constant $c_{0}$. Hence, the above is a non-degenerate parabolic equation. Since w does not vanish on the boundary, we introduce $w=\mathbf{w}-u_{e}-\left[u_{b}-u_{e}\right] e^{-\eta}$. Hence, it follows that $w$ vanishes at both $y=0$ and $y=\infty$, and there holds

$$
w_{x}=\left[\mathbf{w} w_{\eta}\right]_{\eta}-\left[u_{b}-u_{e}\right]\left[w e^{-\eta}\right]_{\eta}-F_{\eta}, \quad F(\eta):=\left[u_{b}-u_{e}\right]\left[u_{e}+\left[u_{e}-u_{b}\right] e^{-\eta}\right] e^{-\eta} .
$$

Clearly, $\langle\eta\rangle^{n} F(\cdot) \in W^{k, p}\left(\mathbb{R}_{+}\right)$, for arbitrary $n, k \geq 0$ and $p \in[1, \infty]$. We shall solve this equation via the standard contraction mapping.

First, let us derive a priori weighted estimates. We introduce the following weighted iterative norm:

$$
\mathcal{N}_{j}(x):=\sum_{k=0}^{j} \int\langle\eta\rangle^{n}\left|\partial_{x}^{k} w\right|^{2}+\sum_{k=0}^{j} \int_{0}^{x} \int\langle\eta\rangle^{n}\left|\partial_{x}^{k} w_{\eta}\right|^{2}, \quad j \geq 0 .
$$

By multiplying the equation (2.9) by $\langle\eta\rangle^{n} w$, it follows the standard weighted energy estimate:

$$
\frac{1}{2} \frac{d}{d x} \int\langle\eta\rangle^{n}|w|^{2}+\int\langle\eta\rangle^{n} \mathbf{w}\left|w_{\eta}\right|^{2} \leq \int\left[\langle\eta\rangle^{n-1}|w|\left|w_{\eta}\right|+\langle\eta\rangle^{n} e^{-\eta}\left|w w_{\eta}\right|+\langle\eta\rangle^{n}\left|w F_{\eta}\right|\right],
$$

for $n \geq 0$, which together with the Young's inequality yields

$$
\frac{1}{2} \frac{d}{d x} \int\langle\eta\rangle^{n}|w|^{2}+\int\langle\eta\rangle^{n} \mathbf{w}\left|w_{\eta}\right|^{2} \leq C \int\langle\eta\rangle^{n}|w|^{2}+C \int\langle\eta\rangle^{n}\left|F_{\eta}\right|^{2} .
$$


The Gronwall inequality then yields

$$
\sup _{0 \leq x \leq L} \int\langle\eta\rangle^{n}|w|^{2}+\int_{0}^{L} \int\langle\eta\rangle^{n}\left|w_{\eta}\right|^{2} \leq C(L),
$$

which gives $\mathcal{N}_{0}(L) \leq C(L)$, for some constant $C(L)$ that depends only on large $L$ and the give data $u_{e}, u_{b}, c_{0}$ in the problem.

Next, taking $x$-derivatives of (2.9), we get

$$
\partial_{x}^{j} w_{x}=\left\{\mathbf{w} \partial_{x}^{j} w_{\eta}\right\}_{\eta}+\sum_{\alpha=0}^{j} C_{j}^{j-\alpha}\left\{\partial_{x}^{j-\alpha} \mathbf{w} \partial_{x}^{\alpha} w_{\eta}\right\}_{\eta}-\left[u_{b}-u_{e}\right]\left[\partial_{x}^{j} w e^{-\eta}\right]_{\eta}
$$

for $j \geq 1$. Similarly as above, multiplying the equation by $\langle\eta\rangle^{n} \partial_{x}^{j} w$ yields the inequality

$$
\begin{aligned}
& \frac{1}{2} \frac{d}{d x} \int\langle\eta\rangle^{n}\left|\partial_{x}^{j} w\right|^{2}+\int\langle\eta\rangle^{n} \mathbf{w}\left|\partial_{x}^{j} w_{\eta}\right|^{2} \\
\leq & n \int\langle\eta\rangle^{n-1} \mathbf{w} \partial_{x}^{j} w_{\eta} \partial_{x}^{j} w+C \sum_{\alpha<j} \int\langle\eta\rangle^{n}\left\{\partial_{x}^{j-\alpha} \mathbf{w} \partial_{x}^{\alpha} w_{\eta}\right\} \partial_{x}^{j} w_{\eta} \\
& +C \sum_{\alpha<j} \int\langle\eta\rangle^{n-1}\left\{\partial_{x}^{j-\alpha} \mathbf{w} \partial_{x}^{\alpha} w_{\eta}\right\} \partial_{x}^{j} w-\left[u_{b}-u_{e}\right] \int\left[\partial_{x}^{j} w e^{-\eta}\right]_{\eta}\langle\eta\rangle^{n} \partial_{x}^{j} w .
\end{aligned}
$$

Let us treat each term on the right. For arbitrary positive constant $\delta$, we get

$$
\begin{aligned}
\int\langle\eta\rangle^{n-1} \mathbf{w} \partial_{x}^{j} w_{\eta} \partial_{x}^{j} w & \leq \delta \int \mathbf{w}\langle\eta\rangle^{n}\left|\partial_{x}^{j} w_{\eta}\right|^{2}+C_{\delta}\|\mathbf{w}\|_{\infty} \int\langle\eta\rangle^{n}\left|\partial_{x}^{j} w\right|^{2} \\
\int\left[\partial_{x}^{j} w e^{-\eta}\right]_{\eta}\langle\eta\rangle^{n} \partial_{x}^{j} w & \leq \delta \int \mathbf{w}\langle\eta\rangle^{n}\left|\partial_{x}^{j} w_{\eta}\right|^{2}+C_{\delta} \int\langle\eta\rangle^{n}\left|\partial_{x}^{j} w\right|^{2} .
\end{aligned}
$$

By choosing $\delta$ sufficiently small, the first term in the above inequalities can be absorbed into the left-hand side of the inequality (2.12). Next, for $0<\alpha<j$, we have

$$
\begin{aligned}
\int\langle\eta\rangle^{n}\left\{\partial_{x}^{j-\alpha} \mathbf{w} \partial_{x}^{\alpha} w_{\eta}\right\} \partial_{x}^{j} w_{\eta} & \leq \delta \int \mathbf{w}\langle\eta\rangle^{n}\left|\partial_{x}^{j} w_{\eta}\right|^{2}+C_{\delta}\left\|\partial_{x}^{j-\alpha} \mathbf{w}\right\|_{\infty}^{2} \int\langle\eta\rangle^{n} \mathbf{w}\left|\partial_{x}^{\alpha} w_{\eta}\right|^{2} \\
\int\langle\eta\rangle^{n-1}\left\{\partial_{x}^{j-\alpha} \mathbf{w} \partial_{x}^{\alpha} w_{\eta}\right\} \partial_{x}^{j} w & \leq C\left\|\partial_{x}^{j-\alpha} \mathbf{w}\right\|_{\infty}\left\|\sqrt{\langle\eta\rangle^{n} \mathbf{w}} \partial_{x}^{\alpha} w_{\eta}\right\|_{L^{2}\left(\mathbb{R}_{+}\right)}\left\|\langle\eta\rangle^{\frac{n-2}{2}} \partial_{x}^{j} w\right\|_{L^{2}\left(\mathbb{R}_{+}\right)} \\
& \leq \delta \int \mathbf{w}\langle\eta\rangle^{n}\left|\partial_{x}^{\alpha} w_{\eta}\right|^{2}+C_{\delta}\left\|\partial_{x}^{j-\alpha} \mathbf{w}\right\|_{\infty}^{2} \int\langle\eta\rangle^{n}\left|\partial_{x}^{j} w\right|^{2}
\end{aligned}
$$

Whereas in the case $\alpha=0$, we instead estimate

$$
\begin{aligned}
\int\langle\eta\rangle^{n}\left\{\partial_{x}^{j} \mathbf{w} w_{\eta}\right\} \partial_{x}^{j} w_{\eta} & \leq \delta \int \mathbf{w}\langle\eta\rangle^{n}\left|\partial_{x}^{j} w_{\eta}\right|^{2}+C_{\delta}\left\|w_{\eta}\right\|_{\infty}^{2} \int\langle\eta\rangle^{n}\left|\partial_{x}^{j} \mathbf{w}\right|^{2} \\
\int\langle\eta\rangle^{n-1}\left\{\partial_{x}^{j} \mathbf{w} w_{\eta}\right\} \partial_{x}^{j} w & \leq\left\|w_{\eta}\right\|_{\infty}\left\|\sqrt{\langle\eta\rangle^{n}} \partial_{x}^{j} \mathbf{w}\right\|_{L^{2}\left(\mathbb{R}_{+}\right)}\left\|\langle\eta\rangle^{\frac{n-2}{2}} \partial_{x}^{j} w\right\|_{L^{2}\left(\mathbb{R}_{+}\right)} .
\end{aligned}
$$

It remains to give bounds on $\left\|w_{\eta}\right\|_{\infty}$ and $\left\|\partial_{x}^{j-\alpha} \mathbf{w}\right\|_{\infty}$, for $0<\alpha \leq j$. We recall the definition $\mathbf{w}=w+u_{e}+\left[u_{b}-u_{e}\right] e^{-\eta}$. Using the Sobolev embedding, we get

$$
\begin{aligned}
\left\|\partial_{x}^{j-\alpha} \mathbf{w}\right\|_{\infty}^{2} & \leq C+\left\|\partial_{x}^{j-\alpha} w\right\|_{\infty}^{2} \leq C+\left\|\partial_{x}^{j-\alpha} w\right\|_{L^{2}\left(\mathbb{R}_{+}\right)}\left\|\partial_{x}^{j-\alpha} w_{\eta}\right\|_{L^{2}\left(\mathbb{R}_{+}\right)} \\
& \leq C+\left\|\partial_{x}^{j-\alpha} w\right\|_{L^{2}\left(\mathbb{R}_{+}\right)}\left[\left\|\partial_{x}^{j-\alpha} w_{\left.\right|_{x=0}}\right\|_{L^{2}\left(\mathbb{R}_{+}\right)}+\left\|\partial_{x}^{j-\alpha} w_{\eta}\right\|_{L^{2}}^{1 / 2}\left\|\partial_{x}^{j-\alpha+1} w_{\eta}\right\|_{L^{2}}^{1 / 2}\right]
\end{aligned}
$$


in which $\|\cdot\|_{L^{2}}$ denotes the usual $L^{2}$ norm over $[0, x] \times \mathbb{R}_{+}$. In term of the iterative norm $\mathcal{N}_{j}(x)$, this yields

$$
\left\|\partial_{x}^{j-\alpha} \mathbf{w}\right\|_{\infty}^{2} \leq C+\mathcal{N}_{j-\alpha}^{1 / 2}(x)\left[\left\|\partial_{x}^{j-\alpha} w_{\eta_{x=0}}\right\|_{L^{2}\left(\mathbb{R}_{+}\right)}+\mathcal{N}_{j}^{1 / 2}(x)\right]
$$

for $0<\alpha \leq j$. Next, to estimate $\left\|w_{\eta}\right\|_{\infty}$, we use the embedding: $\left\|w_{\eta}\right\|_{\infty}^{2} \leq C\left\|w_{\eta}\right\|_{L^{2}\left(\mathbb{R}_{+}\right)}\left\|w_{\eta}\right\|_{H^{1}\left(\mathbb{R}_{+}\right)}$. Using the equation (2.9) and the lower bound on $\mathbf{w}$, we get

$$
\left\|w_{\eta \eta}\right\|_{L^{2}\left(\mathbb{R}_{+}\right)} \leq C\left(\left\|w_{x}\right\|_{L^{2}\left(\mathbb{R}_{+}\right)}+\|w\|_{H^{1}\left(\mathbb{R}_{+}\right)}+\left\|w_{\eta}^{2}\right\|_{L^{2}\left(\mathbb{R}_{+}\right)}+\left\|F_{\eta}\right\|_{L^{2}\left(\mathbb{R}_{+}\right)}\right)
$$

in which the Sobolev embedding for the supremum norm again yields

$$
\left\|w_{\eta}^{2}\right\|_{L^{2}\left(\mathbb{R}_{+}\right)} \leq C\left\|w_{\eta}\right\|_{L^{2}\left(\mathbb{R}_{+}\right)}^{3 / 2}\left\|w_{\eta}\right\|_{H^{1}}^{1 / 2} \leq \delta\left\|w_{\eta \eta}\right\|_{L^{2}\left(\mathbb{R}_{+}\right)}+C_{\delta}\left\|w_{\eta}\right\|_{L^{2}\left(\mathbb{R}_{+}\right)}^{3} .
$$

Choosing $\delta$ sufficiently small, we conclude that

$$
\left\|w_{\eta}\right\|_{\infty} \lesssim 1+\left\|w_{x}\right\|_{L^{2}\left(\mathbb{R}_{+}\right)}+\|w\|_{H^{1}\left(\mathbb{R}_{+}\right)}+\left\|w_{\eta}\right\|_{L^{2}\left(\mathbb{R}_{+}\right)}^{2} .
$$

Hence, integrating the above inequality over $[0, x]$, recalling the definition of the iterative norm, and using the uniform bound on $\mathcal{N}_{0}(L)$, we obtain

$$
\begin{aligned}
\int_{0}^{x}\left\|w_{\eta}\right\|_{\infty}^{2} & \lesssim 1+\int_{0}^{x}\left(\left\|w_{x}\right\|_{L^{2}\left(\mathbb{R}_{+}\right)}^{2}+\|w\|_{H^{1}\left(\mathbb{R}_{+}\right)}^{2}\right)+\sup _{s \in[0, x]}\left\|w_{\eta}\right\|_{L^{2}\left(\mathbb{R}_{+}\right)}^{2} \int_{0}^{x}\left\|w_{\eta}\right\|_{L^{2}\left(\mathbb{R}_{+}\right)}^{2} \\
& \lesssim 1+\int_{0}^{x}\left\|w_{x}\right\|_{L^{2}\left(\mathbb{R}_{+}\right)}^{2}+\left\|w_{\eta_{x=0}}\right\|_{L^{2}\left(\mathbb{R}_{+}\right)}^{2}+\left\|w_{x \eta}\right\|_{L^{2}}^{2} \\
& \lesssim 1+\left\|w_{\eta_{x=0}}\right\|_{L^{2}\left(\mathbb{R}_{+}\right)}^{2}+\int_{0}^{x} \mathcal{N}_{1}(s) d s .
\end{aligned}
$$

Putting the above estimates altogether into the $j^{\text {th }}$ weighted estimates (2.12), integrating the result over $[0, x]$ and rearranging terms, we obtain

$$
\begin{aligned}
N_{j}(x) \lesssim & \int_{0}^{x}\left(1+\|\mathbf{w}\|_{\infty}+\left\|w_{\eta}\right\|_{\infty}^{2}\right) \int\langle\eta\rangle^{n}\left|\partial_{x}^{j} w\right|^{2}+\left\|\partial_{x}^{j-\alpha} \mathbf{w}\right\|_{\infty}^{2} \sum_{\alpha<j} \iint\langle\eta\rangle^{n} \mathbf{w}\left|\partial_{x}^{\alpha} w_{\eta}\right|^{2} \\
& +\left\|\partial_{x}^{j-\alpha} \mathbf{w}\right\|_{\infty} \sum_{\alpha<j}\left\|\sqrt{\langle\eta\rangle^{n} \mathbf{w}} \partial_{x}^{\alpha} w_{\eta}\right\|_{L^{2}}\left\|\langle\eta\rangle^{\frac{n-2}{2}} \partial_{x}^{j} w\right\|_{L^{2}} \\
\lesssim & \mathcal{N}_{j}(0)+\int_{0}^{x}\left[1+\mathcal{N}_{1}(s)+\mathcal{N}_{j-1}(x)\right] \mathcal{N}_{j}(s) d s
\end{aligned}
$$

The Gronwall inequality then yields

$$
\mathcal{N}_{j}(L) \leq C \mathcal{N}_{j}(0), \quad \forall j \geq 0,
$$

for $L$ sufficiently small. Here, we note that the smallness of $L$ and the constant $C$ depend only on the given data in the problem. The standard contraction mapping, together with a priori bounds, yields the existence and the uniform bound of the solutions to (2.9) in $[0, L] \times \mathbb{R}_{+}$. Changing back to the original coordinates yields the lemma, upon noting that $y \sim \eta$ thanks to the upper and lower bound of $\mathbf{w}$. 
Remark 2.4. It is possible to iterate our above scheme to obtain a global-in- $x$ solution to the Prandtl equation. Indeed, the $L^{2}$ estimate (2.11) yields the global existence of a bounded weak solution. The standard Nash-Moser's iteration applied to the parabolic equation (2.9) then yields a uniform bound in $C^{1}$ and hence $H^{1}$ spaces. By a view of the iterative estimate (2.16) which is only nonlinear at the first step for $\mathcal{N}_{1}$, it follows a uniform bound for all $\mathcal{N}_{k}$, for $k \geq 1$, uniformly in small $L$. This yields the global smooth solution.

\section{$2.2 \quad \varepsilon^{1 / 2}$-order corrections}

Next, we collect all terms with a factor $\sqrt{\varepsilon}$ from (2.2a), together with the new $\sqrt{\varepsilon}$-order terms arising from $R^{u, 0}$ (see (2.5)), to get

$$
\begin{aligned}
R^{u, 1}:= & {\left[u_{e}^{1}+u_{p}^{1}\right] \partial_{x}\left[u_{e}^{0}+u_{p}^{0}\right]+\left[u_{e}^{0}+u_{p}^{0}\right] \partial_{x}\left[u_{e}^{1}+u_{p}^{1}\right]+v_{p}^{1} \partial_{y}\left[u_{e}^{0}+u_{p}^{0}\right]+\left[v_{p}^{0}+v_{e}^{1}\right] \partial_{y}\left[u_{e}^{1}+u_{p}^{1}\right] } \\
& +p_{e x}^{1}+p_{p x}^{1}-\partial_{y}^{2}\left[u_{e}^{1}+u_{p}^{1}\right]+\left[y u_{p x}^{0}+v_{p}^{0}+v_{e}^{1}\right] u_{e z}^{0}+y v_{e z}^{1} u_{p y}^{0} .
\end{aligned}
$$

We construct the Euler and Prandtl layers so that $R^{u, 1}$ is of order $\sqrt{\varepsilon}$. We rearrange terms with respect to the interior variables $(x, \sqrt{\varepsilon} y)$ and the boundary-layer variables $(x, y)$, respectively. We stress that when the partial derivative $\partial_{y}$ hits an interior term with scaling $\sqrt{\varepsilon} y$, that term can be moved to the next order. For instance, $\left[v_{p}^{0}+v_{e}^{1}\right] \partial_{y} u_{e}^{1}=\sqrt{\varepsilon}\left[v_{p}^{0}+v_{e}^{1}\right] u_{e z}^{1}(\sqrt{\varepsilon} y)$. Having this in mind, the leading interior terms consist of

$$
u_{e}^{0} u_{e x}^{1}+v_{e}^{1} u_{e z}^{0}+p_{e x}^{1}=0
$$

and the boundary-layer terms consisting of

$$
\begin{aligned}
{\left[u_{e}^{1}+u_{p}^{1}\right] u_{p x}^{0}+} & u_{p}^{0} u_{e x}^{1}+\left[u_{e}^{0}+u_{p}^{0}\right] u_{p x}^{1}+v_{p}^{1}\left[u_{p y}^{0}+\sqrt{\varepsilon} u_{e z}^{0}\right]+\left[v_{p}^{0}+v_{e}^{1}\right] u_{p y}^{1}+p_{p x}^{1}-u_{p y y}^{1} \\
& +\left[y u_{p x}^{0}+v_{p}^{0}\right] u_{e z}^{0}+y v_{e z}^{1} u_{p y}^{0}=0,
\end{aligned}
$$

in which the equalities are made to precisely get rid of these leading terms. Hence, having constructed these layers, the error is then reduced to

$$
\sqrt{\varepsilon}\left[v_{p}^{0}+v_{e}^{1}\right] u_{e z}^{1}-\varepsilon u_{e z z}^{1} .
$$

Next, let us consider the normal component (2.2b). Clearly, the leading term is $\frac{1}{\sqrt{\varepsilon}} p_{p y}^{1}$, which leads to the fact that Prandtl's pressure is independent of $y$ :

$$
p_{p}^{1}=p_{p}^{1}(x)
$$

The next (zeroth) order in (2.2b) consists of

$$
R^{v, 0}:=\left[u_{e}^{0}+u_{p}^{0}\right]\left[v_{p x}^{0}+v_{e x}^{1}\right]+\left[v_{p}^{0}+v_{e}^{1}\right] \partial_{y}\left[v_{p}^{0}+v_{e}^{1}\right]+p_{e z}^{1}+p_{p y}^{2}-\partial_{y}^{2}\left[v_{p}^{0}+v_{e}^{1}\right] .
$$

Again as above, we shall enforce $R^{v, 0}=0$ (possibly, up to error of order $\sqrt{\varepsilon}$ ). Note that $v_{p}^{1}$ has now been determined through the divergence-free condition and the construction of $u_{p}^{1}$. We take the interior layer $\left[u_{e}^{1}, v_{e}^{1}, p_{e}^{1}\right]$ to satisfy

$$
u_{e}^{0} v_{e x}^{1}+p_{e z}^{1}=0 .
$$


Whereas, the next layer pressure $p_{p}^{2}$ is taken to be of the form

$$
p_{p}^{2}(x, y)=\int_{y}^{\infty}\left[\left[u_{e}^{0}+u_{p}^{0}\right] v_{p x}^{0}+u_{p}^{0} v_{e x}^{1}+\left[v_{p}^{0}+v_{e}^{1}\right]\left[v_{p y}^{0}+\sqrt{\varepsilon} v_{e z}^{1}\right]-v_{p y y}^{0}-\varepsilon v_{e z z}^{1}\right](x, \theta) d \theta .
$$

With this choice of $p_{p}^{2}$ and (2.21), the error term $R^{v, 0}$ in this leading order is reduced to

$$
R^{v, 0}=\sqrt{\varepsilon}\left[v_{p}^{0}+v_{e}^{1}\right] v_{e z}^{1}-\varepsilon v_{e z z}^{1} .
$$

The set of equations (2.17), (2.21), together with the divergence-free condition, constitutes the profile equations for the Euler correction $\left[u_{e}^{1}, v_{e}^{1}, p_{e}^{1}\right]$, whereas equations (2.18) and (2.20) are for the divergence-free Prandtl layers $\left[u_{p}^{1}, v_{p}^{1}, p_{p}^{1}\right]$.

\section{$2.3 \quad$ Euler correctors}

We construct the Euler corrector $\left[v_{e}^{1}, u_{e}^{1}, p_{e}^{1}\right]$ solving (2.17), (2.21). For sake of presentation, we drop the superscript 1 . Writing the equation for vorticity $w_{e}=u_{e z}-v_{e x}$ and note that $w_{e x}=-\Delta v_{e}$. This leads to the following elliptic problem for $v_{e}$ :

$$
-u_{e}^{0} \Delta v_{e}+u_{e z z}^{0} v_{e}=0
$$

with $\Delta=\partial_{x}^{2}+\partial_{z}^{2}$, together with the boundary conditions as in Section 1.1, which we recall

$$
v_{e}(x, 0)=-v_{p}^{0}(x, 0), \quad v_{e}(0, z)=V_{b 0}(z), \quad v_{e}(L, z)=V_{b L}(z)
$$

with the comparability assumption: $V_{b 0}(0)=-v_{p}^{0}(0,0)$ and $V_{b L}(0)=-v_{p}^{0}(L, 0)$. We need to derive higher regularity estimates for $v_{e}$. Due to the presence of corners in the domain for $(x, z)$, singularity could occur. To avoid this, we instead consider the following elliptic problem:

$$
-u_{e}^{0} \Delta v_{e}+u_{e z z}^{0} v_{e}=E_{b},
$$

with the same boundary conditions (2.25), in which $E_{b}$ is introduced as a boundary layer corrector. To define $E_{b}$, let us introduce

$$
\begin{aligned}
B(x, z) & :=\left(1-\frac{x}{L}\right) \frac{V_{b 0}(z)}{v_{p}^{0}(0,0)} v_{p}^{0}(x, 0)+\frac{x}{L} \frac{V_{b L}(z)}{v_{p}^{0}(L, 0)} v_{p}^{0}(x, 0) \\
& =\frac{V_{b 0}(z)}{v_{p}^{0}(0,0)} v_{p}^{0}(x, 0)+\frac{x}{L}\left(\frac{V_{b L}(z)}{v_{p}^{0}(L, 0)}-\frac{V_{b 0}(z)}{v_{p}^{0}(0,0)}\right) v_{p}^{0}(x, 0) .
\end{aligned}
$$

Here, without loss of generality, assume that $v_{p}^{0}(0,0), v_{p}^{0}(L, 0) \neq 0$. It is clear that $B(x, z)$ satisfies the boundary conditions (2.25), thanks to the compatibility assumption at the corners. In addition, if we assume $\left|\partial_{z}^{k}\left(V_{b L}-V_{b 0}\right)(z)\right| \leq C L$, it follows that $B \in W^{k, p}$ for arbitrary $k, p$. In particular, the function

$$
F_{e}(x, z):=-u_{e}^{0} \Delta B+u_{e z z}^{0} B
$$

is arbitrarily smooth and there holds

$$
\left\|\langle z\rangle^{n} F_{e}\right\|_{W^{k, q}} \leq C
$$


for any $n, k \geq 0$ and $q \in[1, \infty]$, for some constant $C$ that is independent of small $L$. Let us then introduce the function $w$ through

$$
v_{e}=B+w
$$

The function $w$ solves the following elliptic problem with homogenous boundary conditions:

$$
-u_{e}^{0} \Delta w+u_{e z z}^{0} w=E_{b}+F_{e}(x, z), \quad w_{\mid \partial \Omega}=0 .
$$

To obtain high regularity for $w$, we introduce the boundary layer corrector:

$$
E_{b}(x, z):=-\chi\left(\frac{z}{\varepsilon}\right) F_{e}(x, 0),
$$

in which $\chi(\cdot)$ is a cut-off function with support in $[0,1]$ and with $\chi(0)=1$. It follows that

$$
\left\|\langle z\rangle^{n} \partial_{z}^{k} E_{b}\right\|_{L^{q}} \leq C \varepsilon^{-k+\frac{1}{q}}, \quad q \geq 1, \quad n, k \geq 0 .
$$

Let us now derive sufficient estimates on $v_{e}$. We prove the following:

Lemma 2.5. Assume that $V_{b 0}$ and $V_{b L}$ are sufficiently smooth, rapidly decaying at infinity, and satisfy $\left|\partial_{z}^{k}\left(V_{b L}-V_{b 0}\right)(z)\right| \leq C L$, for $k \geq 0$, uniformly in $z$. There exists a unique smooth solution $v_{e}$ to the elliptic problem (2.25), (2.26), and (2.30), and there holds

$$
\left\|v_{e}\right\|_{\infty}+\left\|\langle z\rangle^{n} v_{e}\right\|_{H^{2}} \leq C_{0}, \quad\left\|\langle z\rangle^{n} v_{e}\right\|_{H^{3}} \leq C_{0} \varepsilon^{-1 / 2}, \quad\left\|\langle z\rangle^{n} v_{e}\right\|_{H^{4}} \leq C_{0} \varepsilon^{-3 / 2}
$$

for $n \geq 0$ and for some constant $C_{0}$ that depends on the given boundary data, and but does not depend on $L$, when $L$ is small. In addition,

$$
\left\|\langle z\rangle^{n} v_{e}\right\|_{W^{2, q}} \leq C(L), \quad\left\|\langle z\rangle^{n} v_{e}\right\|_{W^{3, q}} \leq C(L) \varepsilon^{-1+1 / q}, \quad\left\|\langle z\rangle^{n} v_{e}\right\|_{W^{4, q}} \leq C(L) \varepsilon^{-2+1 / q},
$$

for $n \geq 0, q \in(1, \infty)$, and for some constant $C(L)$ that could depend on small L. Here, we note that the integration in the above $\|\cdot\|_{W^{k, q}}$ norms is taken with respect to $(x, z)$ in $[0, L] \times \mathbb{R}_{+}$.

Proof. We write $v_{e}=B+w$ with the smooth $B$ defined as in (2.27). It suffices to derive estimates on $w$, solving (2.29). We first perform the basic $L^{2}$ estimate. Multiplying the equation by $w / u_{e}^{0}$ and using the zero boundary conditions yield

$$
\iint\left(-\Delta w w+\frac{u_{e z z}^{0}}{u_{e}^{0}}|w|^{2}\right)=\iint\left(|\nabla w|^{2}+\frac{u_{e z z}^{0}}{u_{e}^{0}}|w|^{2}\right)=\iint \frac{w\left(E_{b}+F_{e}\right)}{u_{e}^{0}} .
$$

Thanks to the crucial positivity estimate (see (1.16) and (1.17) with $u_{e}^{0}$ ), we have

$$
\int\left(\left|w_{z}\right|^{2}+\frac{u_{e z z}^{0}}{u_{e}^{0}}|w|^{2}\right)=\int\left|u_{e}^{0}\right|^{2}\left\{\frac{w}{u_{e}^{0}}\right\}_{z}^{2} \geq \theta_{0} \int\left|w_{z}\right|^{2}
$$

in which we have used $\left|w / u_{0}^{e}\right| \leq \sqrt{z}\left\|\left(w / u_{e}^{0}\right)_{z}\right\|_{L^{2}\left(\mathbb{R}_{+}\right)}$and the fast decay property of $u_{e z}^{0}$ to obtain the lower bound, for some constant $\theta_{0}$ independent of $L$. In addition, we estimate

$$
\iint \frac{w\left(E_{b}+F_{e}\right)}{u_{e}^{0}} \leq \iint \frac{\sqrt{x}\left|E_{b}+F_{e}\right|}{u_{e}^{0}}\left\|w_{x}\right\|_{L^{2}(0, L)} \leq C\left\|E_{b}+F_{e}\right\|_{L^{2}}\|\nabla w\|_{L^{2}} \leq C\|\nabla w\|_{L^{2}} .
$$


Putting the above estimates into the energy estimate, together with a use of the standard Young's inequality, we get

$$
\|w\|_{H^{1}} \leq C
$$

for some constant $C$ that is independent of small $L$, in which the $L^{2}$ norm of $w$ is bounded by the Poincare's inequality.

Next, to derive high order energy estimates, we write the equation as

$$
-\Delta w=G_{e}, \quad G_{e}:=\frac{1}{u_{e}^{0}}\left(E_{b}+F_{e}-u_{e z z}^{0} w\right) .
$$

Clearly, $\left\|G_{e}\right\|_{L^{2}} \leq C$. In addition, since $E_{b}(x, 0)+F_{e}(x, 0)=0$, we have $G_{e}=0$ and hence $w=w_{z z}=0$ on the boundary $z=0$. We thus have the following $H^{2}$ energy estimate by multiplying the elliptic equation by $w_{z}$ :

$$
\iint\left|\nabla w_{z}\right|^{2}+\int_{0}^{L} w_{z z} w_{z}(x, 0)+\left.\int_{0}^{\infty} w_{z x} w_{z}\right|_{x=0} ^{x=L}=\iint\left(G_{e}\right)_{z} w_{z}=-\iint G_{e} w_{z z} \leq\left\|G_{e}\right\|_{L^{2}}\left\|w_{z z}\right\|_{L^{2}} .
$$

Since $w_{z z}(x, 0)=0$ and $w_{z}(0, z)=w_{z}(L, z)=0$, the boundary terms vanish. Hence, together with a use of the Young's inequality, we have obtained the uniform bound $\left\|w_{z}\right\|_{H^{1}} \leq C$. Using the equation to estimate $w_{x x}$ in term of the rest, we thus obtain the full $H^{2}$ bound of the solution $w$, and hence of $v_{e}$, uniformly in small $L$. In addition, since $w=0$ on the boundary, we have

$$
\begin{aligned}
|w(x, z)| & \leq \int_{0}^{x}\left|w_{x}(\theta, z)\right| d \theta \leq \int_{0}^{x}\left(\int_{0}^{z}\left|w_{x} w_{x z}\right|(\theta, \eta) d \eta\right)^{1 / 2} d \theta \\
& \leq \sqrt{x}\left\|w_{x}\right\|_{L^{2}}^{1 / 2}\left\|w_{x z}\right\|_{L^{2}}^{1 / 2} \leq C_{0} \sqrt{L},
\end{aligned}
$$

thanks to the $H^{2}$ bound on $w$. This proves the uniform boundedness of $v_{e}$.

As for the weighted estimates, we consider the elliptic problem for $\langle z\rangle^{n} w$, with $n \geq 1$, which solves

$$
-\Delta\left(\langle z\rangle^{n} w\right)=\frac{\langle z\rangle^{n}}{u_{e}^{0}}\left(E_{b}+F_{e}-u_{e z z}^{0} w\right)-w \partial_{z}^{2}\langle z\rangle^{n}-2 w_{z} \partial_{z}\langle z\rangle^{n}
$$

the homogenous boundary conditions. By induction, $\langle z\rangle^{n-1} w$ is uniformly bounded in $H^{2}$, and hence the right-hand side of the above elliptic problem is uniformly bounded in $H^{1}$. The same proof given just above for the unweighted norm yields $\left\|\langle z\rangle^{n} w\right\|_{H^{2}} \leq C$, for all $n \geq 1$.

Next, we derive higher regularity estimates for $w$. We recall that from (2.28) and (2.31), there holds

$$
\left\|\langle z\rangle^{n} \partial_{z}^{k}\left(E_{b}+F_{e}\right)\right\|_{L^{q}} \leq C\left(1+\varepsilon^{-k+\frac{1}{q}}\right), \quad q \geq 1, \quad n, k \geq 0 .
$$

Let us now consider the elliptic problems for $w_{z}$ and $w_{z z}$ :

$$
-\Delta w_{z}=\partial_{z}\left[\frac{1}{u_{e}^{0}}\left(F_{e}+E_{b}-u_{e z z}^{0} w\right)\right], \quad w_{\left.z\right|_{x=0, L}}=w_{\left.z z\right|_{z=0}}=0
$$

and

$$
-\Delta w_{z z}=\partial_{z}^{2}\left[\frac{1}{u_{e}^{0}}\left(F_{e}+E_{b}-u_{e z z}^{0} w\right)\right], \quad w_{\left.z z\right|_{\partial \Omega}}=0
$$


Here, we note that $w_{z z}=0$ on the boundary, precisely due to the layer corrector $E_{b}$ and the equation (2.33). Next, note that the source term in the above equations has its $L^{2}$ norm bounded by $C \varepsilon^{-1 / 2}$ and $C \varepsilon^{-3 / 2}$, respectively. Again, the above $H^{2}$ energy estimates then give

$$
\left\|\langle z\rangle^{n} \partial_{z}^{k} w\right\|_{H^{2}} \leq C \varepsilon^{-k+1 / 2}, \quad k=1,2, \quad n \geq 0 .
$$

We now estimate $w$ in $H^{3}$ and $H^{4}$ norms. Indeed, thanks to the $H^{2}$ bound on $w_{z}, w_{z z}$, it remains to estimate $w_{x x x}$ in $L^{2}$ and $H^{1}$, respectively. Thanks to (2.33), we may write

$$
w_{x x x}=-w_{z z x}+\Delta w_{x}=-w_{z z x}-\partial_{x}\left[\frac{1}{u_{e}^{0}}\left(F_{e}+E_{b}-u_{e z z}^{0} w\right)\right] .
$$

This yields at once the desired weighted $L^{2}$ and $H^{1}$ estimates on $w_{x x x}$, and hence the full weighted $H^{3}$ and $H^{4}$ estimates on $w$.

Finally, the $W^{k, q}$ estimates follow simply from the standard elliptic theory in $[0, L] \times \mathbb{R}$, when we make the odd extension to $z<0$ for (2.33). We note that the boundary layer construction (2.30) ensures that the odd extension of $G_{e} \in W^{2, q}([0, L] \times \mathbb{R})$. This completes the proof of the lemma.

\subsubsection{Euler profiles}

We now construct the Euler corrector $\left[u_{e}^{1}, v_{e}^{1}, p_{e}^{1}\right]$ that is used in the boundary-layer expansion (1.9)). We take $v_{e}^{1}=v_{e}$, where $v_{e}$ solves the modified elliptic problem (2.26), with an extra source $E_{b}$. By a view of (2.21) and the divergence-free condition, we take

$$
\begin{aligned}
& u_{e}^{1}:=u_{b}^{1}(z)-\int_{0}^{x} v_{e z}^{1}(\xi, z) d \xi \\
& p_{e}^{1}:=p_{b}-\int_{0}^{x}\left[-u_{e}^{0} v_{e z}^{1}+v_{e}^{1} u_{e z}^{0}\right](\xi, 0) d \xi-\int_{0}^{z}\left[u_{e}^{0} v_{e x}^{1}\right](x, \theta) d \theta,
\end{aligned}
$$

for any constant $p_{b}$. Without loss of generality, we take $p_{b}=0$. Clearly, by definition and the uniform $H^{2}$ estimates on $v_{e}^{1}$ (Lemma 2.5), we have

$$
\left\|u_{e}^{1}\right\|_{\infty}+\left\|\langle z\rangle^{n} u_{e}^{1}\right\|_{H^{1}} \leq C_{0}, \quad n \geq 0
$$

By construction, $\left[u_{e}^{1}, v_{e}^{1}, p_{e}^{1}\right]$ solves (2.21), the divergence-free condition, and instead of (2.17), the equation

$$
u_{e}^{0} u_{e x}^{1}+v_{e}^{1} u_{e z}^{0}+p_{e x}^{1}=-\int_{z}^{\infty} E_{b}(x, \theta) d \theta
$$

As compared to (2.17), this contributes a new error term into (2.19), which is now defined as

$$
R^{u, 1}=\sqrt{\varepsilon}\left[v_{p}^{0}+v_{e}^{1}\right] u_{e z}^{1}-\varepsilon u_{e z z}^{1}+\int_{z}^{\infty} E_{b}(x, \theta) d \theta .
$$

To give an estimate on the error term, we first note that throughout the paper we work with the coordinates $(x, y)$, whereas the Euler flows are evaluated at $(x, z)=(x, \sqrt{\varepsilon} y)$. Thanks to Corollary 2.3. the boundedness of $v_{e}$ and (2.35), we have

$$
\left\|\sqrt{\varepsilon}\left[v_{p}^{0}+v_{e}^{1}\right] u_{e z}^{1}\right\|_{L^{2}} \leq \sqrt{\varepsilon}\left\|v_{p}^{0}+v_{e}^{1}\right\|_{\infty}\left\|u_{e z}^{1}(\sqrt{\varepsilon} \cdot)\right\|_{L^{2}} \leq C \varepsilon^{1 / 4}\left\|u_{e z}^{1}(\cdot)\right\|_{L^{2}} .
$$


Similarly, by definition and the estimates from Lemma 2.5, we have

$$
\varepsilon\left\|u_{e z z}^{1}(\sqrt{\varepsilon} \cdot)\right\|_{L^{2}} \leq \varepsilon^{3 / 4}\left\|u_{b z}^{1}\right\|_{L^{2}}+C \varepsilon^{3 / 4}\left\|\langle z\rangle^{n} v_{e z z z}^{1}\right\|_{L^{2}} \leq C \varepsilon^{1 / 4},
$$

and by the estimate (2.31),

$$
\left\|\int_{\sqrt{\varepsilon} y}^{\infty} E_{b}(x, \theta) d \theta\right\|_{L^{2}} \leq C \varepsilon^{-1 / 4}\left\|\langle z\rangle^{n} E_{b}(\cdot)\right\|_{L^{2}} \leq C \varepsilon^{1 / 4}
$$

Hence, we obtain the uniform error estimate:

$$
\left\|R^{u, 1}\right\|_{L^{2}} \leq C \varepsilon^{1 / 4}
$$

Similarly, we give an estimate on $R^{v, 0}$ defined as in (2.23). Thanks to the boundedness of $v_{p}^{0}$ and $v_{e}^{1}$, and the estimates on $v_{e}^{1}$ (Lemma 2.5), we get

$$
\left\|R^{v, 0}\right\|_{L^{2}} \leq \sqrt{\varepsilon}\left\|v_{p}^{0}+v_{e}^{1}\right\|_{L^{\infty}}\left\|v_{e z}^{1}\right\|_{L^{2}}+\varepsilon\left\|v_{e z z}^{1}\right\|_{L^{2}} \leq C \varepsilon^{1 / 4} .
$$

Finally, we estimate $E^{0}$ defined as in (2.3). Using the fact that $u_{p}^{0}$ is rapidly decaying at infinity and $v_{e z z}^{1}$ is in $L^{2}$, we obtain

$$
\left\|E^{0}\right\|_{L^{2}} \leq \varepsilon\left\|\langle y\rangle u_{p x}^{0}\right\|_{L^{2}}\left\|u_{e z z}^{0}\right\|_{L^{\infty}}+\varepsilon\left\|\langle y\rangle u_{p y}^{0}\right\|_{L^{2}}\left\|v_{e z z}^{1}\right\|_{L^{2}} \leq C \varepsilon^{3 / 4} .
$$

\subsection{Prandtl correctors}

In this subsection, we shall construct Prandtl layer $\left[u_{p}^{1}, v_{p}^{1}, p_{p}^{1}\right]$, solving (2.18), (2.20). For convenience, let us denote

$$
u^{0} \equiv u_{e}^{0}(\sqrt{\varepsilon} y)+u_{p}^{0}
$$

After rearranging terms, the equation (2.18) for the $\sqrt{\varepsilon}$-order Prandtl corrector $\left[u_{p}, u_{p}, p_{p}\right]$, after dropping the superscript 1 , becomes

$$
\begin{aligned}
u^{0} u_{p x} & +u_{p} u_{x}^{0}+v_{p} u_{y}^{0}+\left[v_{p}^{0}+v_{e}^{1}\right] u_{p y}-u_{p y y} \\
& =-u_{e z}^{0}\left[y u_{p x}^{0}+v_{p}^{0}\right]-y v_{e z}^{1} u_{p y}^{0}-u_{e}^{1} u_{p x}^{0}-u_{p}^{0} u_{e x}^{1}-p_{p x} \\
& =: F_{p}
\end{aligned}
$$

in which we note that the source term $F_{p}$ includes the unknown pressure $p_{p}$. Thanks to (2.20), $p_{p}=p_{p}(x)$ and hence, by evaluating the equation (2.41) at $y=\infty$, we get $p_{p x}=0$. We shall solve (2.41) together with the divergence-free condition

$$
u_{p x}+v_{p y}=0
$$

and the boundary conditions:

$$
u_{p}(0, y)=\bar{u}_{1}(y), \quad u_{p}(x, 0)=-u_{e}^{1}(x, 0), \quad v_{p}(x, 0)=0, \quad \lim _{y \rightarrow \infty} u_{p}(x, y)=0 .
$$




\subsubsection{Construction of Prandtl layers}

There is a natural energy estimate associated with the linearized Prandtl equation (2.41), yielding bound on $u_{p y}$ in term of $v_{p}$. The difficulty is in controlling the unknown $v_{p}$. Our construction starts with the crucial positivity estimate (1.16). Indeed, we introduce the inner product

$$
[[v, w]] \equiv \int_{\mathbb{R}_{+}}\left[v_{y} w_{y}+\frac{u_{y y}^{0}}{u^{0}} v w\right] d y .
$$

Note that by (1.16) the quantity $[[v, v]]$ yields a bound on $\left\|v_{y}\right\|_{L^{2}\left(\mathbb{R}_{+}\right)}^{2}$. We therefore shall re-write the equation in term of $v_{p}$, putting $u_{p}$ in the source term. Precisely, taking $y$-derivative of (2.41) and using the divergence-free condition yield

$$
-u^{0} v_{p y y}+v_{p} u_{y y}^{0}+u_{p} u_{x y}^{0}+\left[v_{p}^{0}+v_{e}^{1}\right] u_{p y y}+\sqrt{\varepsilon} v_{e z}^{1} u_{p y}-u_{p y y y}=F_{p y},
$$

or equivalently, in a view of the inner product,

$$
\begin{aligned}
& -v_{p y y}+\frac{u_{y y}^{0}}{u^{0}} v_{p}-\partial_{y}^{2}\left(\frac{u_{p y}}{u^{0}}\right) \\
& =\frac{1}{u^{0}}\left(F_{p y}-u_{p} u_{x y}^{0}-\left[v_{p}^{0}+v_{e}^{1}\right] u_{p y y}-\sqrt{\varepsilon} v_{e z}^{1} u_{p y}\right)-2 u_{p y y}\left(\frac{1}{u^{0}}\right)_{y}-u_{p y}\left(\frac{1}{u^{0}}\right)_{y y} \\
& =: G_{p} .
\end{aligned}
$$

Furthermore, taking $x$-derivative of (2.42) yields

$$
-v_{p x y y}+\frac{u_{y y}^{0}}{u^{0}} v_{p x}+\left(\frac{v_{p y y}}{u^{0}}\right)_{y y}=G_{p x}-\left(\frac{u_{y y}^{0}}{u^{0}}\right)_{x} v_{p}+\left(u_{p y}\left(\frac{1}{u^{0}}\right)_{x}\right)_{y y}
$$

with $G_{p}$ defined as in (2.42). We shall solve the problem (2.42)-(2.43) for $v_{p}$, with $u_{p x}+v_{p y}=0$, and the boundary conditions:

$$
v_{p}(x, 0)=0, \quad v_{p y}(x, 0)=u_{e x}^{1}(x, 0) .
$$

We prove the following:

Lemma 2.6. There exists a unique smooth divergence-free solution $\left[u_{p}, v_{p}\right]$ solving (2.41) with initial condition $u_{p}(0, y)=\bar{u}_{1}(y)$ and the boundary conditions (2.44). Furthermore, there hold

$$
\left\|\left[u_{p}, v_{p}\right]\right\|_{L^{\infty}}+\sup _{0 \leq x \leq L}\left\|\langle y\rangle^{n} v_{p y y}\right\|_{L^{2}\left(\mathbb{R}_{+}\right)}+\left\|\langle y\rangle^{n} v_{p x y}\right\|_{L^{2}} \lesssim C(L, \kappa) \varepsilon^{-\kappa},
$$

for arbitrary small $\kappa$, and high regularity estimates

$$
\sup _{0 \leq x \leq L}\left\|\langle y\rangle^{n} v_{p x y y}\right\|_{L^{2}\left(\mathbb{R}_{+}\right)}+\left\|\langle y\rangle^{n} v_{p x x y}\right\|_{L^{2}} \lesssim C(L) \varepsilon^{-1},
$$

uniformly in small $\varepsilon, L$, in which the bounds depend only on the constructed profiles $\left[u^{0}, v_{p}^{0}\right]$, the given boundary data, and small $L$.

The proof consists of several steps. First, we express the boundary conditions of $v_{p}$ in term of the given data $u_{p}(0, y)=\bar{u}_{1}(z)$. 
Lemma 2.7. For smooth solutions $\left[u_{p}, v_{p}\right]$ solving (2.41) with $u_{p}(0, y)=\bar{u}_{1}(y)$. Then, there hold

$$
\begin{aligned}
\left\|\langle y\rangle^{n} v_{p}(0, \cdot)\right\|_{\dot{H}^{k+1}\left(\mathbb{R}_{+}\right)} & \leq C_{0}\left(1+\left\|\langle y\rangle^{n} \bar{u}_{1}\right\|_{H^{k+3}\left(\mathbb{R}_{+}\right)}\right) \\
\left\|\langle y\rangle^{n} v_{p x}(0, \cdot)\right\|_{\dot{H}^{k+1}\left(\mathbb{R}_{+}\right)} & \leq C_{0}\left(1+\left\|\langle y\rangle^{n} \bar{u}_{1}\right\|_{H^{k+3}\left(\mathbb{R}_{+}\right)}+\left\|\langle y\rangle^{-m} u_{e x x}^{1}(0, \sqrt{\varepsilon} \cdot)\right\|_{H^{k}\left(\mathbb{R}_{+}\right)}\right)
\end{aligned}
$$

for $n, m, k \geq 0$, for some constant $C_{0}=C_{0}\left(u^{0}, v_{p}^{0},\left[u_{e}^{1}, v_{e}^{1}\right](0, \cdot)\right)$ depending only on the profile $\left[u^{0}, v_{p}^{0}\right]$ and given data $\left[u_{e}^{1}, v_{e}^{1}\right](0, \cdot)$.

Proof. Define the stream function $\psi=\int_{0}^{y} u_{p} d y$. Then, $u_{p}=\psi_{y}$ and $v_{p}=-\psi_{x}$. We introduce the quantity $w=u_{0} \psi_{y}-u_{y}^{0} \psi$. Using (2.41), we get

$$
w_{x}=-u_{x y}^{0} \psi-\left[v_{p}^{0}+v_{e}^{1}\right] u_{p y}+u_{p y y}+F_{p},
$$

for $F_{p}$ defined as in (2.41). Hence, the boundary values of $w$ and $w_{x}$ can be computed directly from the given boundary data $\bar{u}_{1}(y),\left[u_{p}^{0}, v_{p}^{0}\right]$, and $F_{p}(0, y)$. Precisely, for $k \geq 0$, we get

$\left\|\partial_{y}^{k} w(0, \cdot)\right\|_{L^{2}} \leq C\left(u^{0}, v_{p}^{0}\right)\left\|\bar{u}_{1}\right\|_{H^{k}\left(\mathbb{R}_{+}\right)}, \quad\left\|\partial_{y}^{k} w_{x}(0, \cdot)\right\|_{L^{2}} \leq C\left(u^{0}, v_{p}^{0}\right)\left\|\bar{u}_{1}\right\|_{H^{k+2}\left(\mathbb{R}_{+}\right)}+\left\|\partial_{y}^{k} F_{p}(0, \cdot)\right\|_{L^{2}\left(\mathbb{R}_{+}\right)}$

for some constant $C\left(u^{0}, v_{p}^{0}\right)$ that depends on the high regularity norms of $u^{0}, v_{p}^{0}$; see the estimates on $\left[u_{p}^{0}, v_{p}^{0}\right]$ in Corollary 2.3. By definition of $F_{p}$ in (2.41) and the fact that $u_{e}^{1}, v_{e}^{1}$, and hence $\partial_{z}^{k}\left[u_{e}^{1}, v_{e}^{1}, u_{e x}^{1}\right]$, are all given on the boundary $x=0$, we get

$$
\left\|\partial_{y}^{k} F_{p}(0, \cdot)\right\|_{L^{2}\left(\mathbb{R}_{+}\right)} \leq C\left(u^{0}, v_{p}^{0},\left[u_{e}^{1}, v_{e}^{1}\right](0, \cdot)\right),
$$

in which the $y$-decay factor comes directly from the decay property of $u_{p}^{0}$ and $C\left(u^{0}, v_{p}^{0},\left[u_{e}^{1}, v_{e}^{1}\right](0, \cdot)\right)$ depends on high regularity norms of $u^{0}, v_{p}^{0}$, and $\left[u_{e}^{1}, v_{e}^{1}\right](0, \cdot)$.

Next, from the definition of $w$, we can write

$$
\psi=u^{0} \int_{0}^{y} \frac{w(x, \theta)}{\left\{u^{0}\right\}^{2}} d \theta
$$

and so we have

$$
\left\|\langle y\rangle^{n} \partial_{y}^{k+1} v_{p}(0, \cdot)\right\|_{L^{2}\left(\mathbb{R}_{+}\right)}=\left\|\langle y\rangle^{n} \partial_{y}^{k+1} \psi_{x}(0, \cdot)\right\|_{L^{2}\left(\mathbb{R}_{+}\right)} \leq C\left(\|w(0, \cdot)\|_{H^{k}\left(\mathbb{R}_{+}\right)}+\left\|w_{x}(0, \cdot)\right\|_{H^{k}\left(\mathbb{R}_{+}\right)}\right) .
$$

This proves the claimed estimate for $v_{p}(0, \cdot)$ in $\dot{H}^{k+1}\left(\mathbb{R}_{+}\right)$. Next, as for $v_{x}$ estimate, we differentiate (2.47) with respect to $x$ and get

$$
\left\|w_{x x}(0, \cdot)\right\|_{H^{k}\left(\mathbb{R}_{+}\right)} \leq C\left(u^{0}, v_{p}^{0}\right)\left(\left\|\langle y\rangle^{-2} \psi_{x}(0, \cdot)\right\|_{H^{k}\left(\mathbb{R}_{+}\right)}+\left\|v_{p}(0, \cdot)\right\|_{H^{k+3}\left(\mathbb{R}_{+}\right)}\right)+\left\|F_{p x}(0, \cdot)\right\|_{H^{k}\left(\mathbb{R}_{+}\right)} .
$$

Again by definition of $F_{p}$ in (2.41), we have

$$
\left\|F_{p x}(0, y)\right\|_{H^{k}\left(\mathbb{R}_{+}\right)} \leq C\left(u^{0}, v_{p}^{0},\left[u_{e}^{1}, v_{e}^{1}\right](0, \cdot)\right)\left(1+\left\|\langle y\rangle^{-m} u_{e x x}^{1}(0, \cdot)\right\|_{H^{k}\left(\mathbb{R}_{+}\right)}\right)
$$

in which $C\left(u^{0}, v_{p}^{0}, v_{e}^{1}(0, \cdot)\right)$ depends on high regularity norms of $u^{0}, v_{p}^{0}$, and $v_{e}^{1}(0, \cdot)$. Similarly, $v_{p y y}(0, y)$ can be written in term of $u_{p}(0, y), v_{p}(0, y), F_{p y}(0, y)$, according to (2.42). This gives

$$
\left\|v_{p}(0, \cdot)\right\|_{H^{k+3}\left(\mathbb{R}_{+}\right)} \leq C\left(u^{0}, v_{p}^{0},\left[u_{e}^{1}, v_{e}^{1}\right](0, \cdot)\right)\left(1+\left\|v_{p}(0, \cdot)\right\|_{\dot{H}^{k+1}\left(\mathbb{R}_{+}\right)}\right)
$$

This yields estimates on $v_{p x}$ on the boundary as claimed. 
Lemma 2.8. There exists a positive number $L>0$ so that for each $N$ large, the fourth order elliptic equation:

$$
-v_{y y x}+\frac{u_{y y}^{0}}{u^{0}} v_{x}+\left\{\frac{v_{y y}}{u^{0}}\right\}_{y y}=f_{y}+g
$$

on $[0, L] \times[0, N]$ has a unique solution satisfying initial condition $v(0, y)=\bar{v}_{0}(y)$ and boundary conditions: $\left[v, v_{y}\right]=0$ at $y=0, N$, with sources $f, g$ in weighted $L^{2}$ spaces. Furthermore, there holds

$$
\begin{aligned}
\sup _{0 \leq x \leq L} & \left\|\langle y\rangle^{n} \partial_{x}^{j} v_{y y}\right\|_{L^{2}([0, N])}+\left\|\langle y\rangle^{n} \partial_{x}^{j} v_{x y}\right\|_{L^{2}} \\
& \leq C \sum_{k=0}^{1}\left(\left\|\langle y\rangle^{n} \partial_{x}^{k} v_{y y}\right\|_{L^{2}(\{x=0\})}+\left\|\langle y\rangle^{n} \partial_{x}^{k} f\right\|_{L^{2}}+\left\|\langle y\rangle^{n+\frac{3}{2}} \partial_{x}^{k} g\right\|_{L^{2}}\right)
\end{aligned}
$$

for $j=0,1$, for any $n \geq 0$, as long as the right-hand side is finite.

Proof. Let us choose an orthogonal basis $\left\{e^{i}(y)\right\}_{i=1}^{\infty}$ in $H^{2}([0, N])$ with $\left[e^{i}, e_{y}^{i}\right]=0$ at $y=0, N$, for all $i \geq 1$. The orthogonality is obtained with respect to the $[[\cdot]]$ inner product:

$$
\left[\left[e^{i}, e^{j}\right]\right]=\delta_{i j} .
$$

Such an orthogonal basis exists, since [[.]] is equivalent to the usual inner product in $H^{1}([0, N])$. Then, we introduce the weak formulation of (2.48):

$$
\left[\left[v_{x}, e^{i}\right]\right]+\int \frac{v_{y y} e_{y y}^{i}}{u^{0}} d y=\int\left(-f e_{y}^{i}+g e^{i}\right) d y
$$

for all $e^{i}(y), i \geq 1$. Next, for each fixed $k$, we construct an approximate solution in $\operatorname{Span}\left\{e^{i}(y)\right\}_{i=1}^{k}$ defined as

$$
v^{k}(x, y):=\sum_{i=1}^{k} a^{i}(x) e_{i}(y)
$$

for each $x \in[0, L]$. Then, $v^{k}(x \cdot)$ solves

$$
\left[\left[v_{x}^{k}, e^{i}\right]\right]+\int \frac{v_{y y}^{k} e_{y y}^{i}}{u^{0}}=\int\left(-f e_{y}^{i}+g e^{i}\right) d y
$$

which by orthogonality yields a system of ODE equations:

$$
a_{x}^{i}+\sum_{i=1}^{k} a^{j} \int \frac{e_{y y}^{j} e_{y y}^{i}}{u^{0}}=\int\left(-f e_{y}^{i}+g e^{i}\right) d y .
$$

Since $f, g \in L^{2}\left(\mathbb{R}_{+}\right)$, the ODE system has the unique smooth solution $a^{k}$ and hence, $v^{k}$ is defined uniquely and smooth. Multiplying (2.50) by $a_{x}^{i}$ and taking the sum over $i$, we get

$$
\left[\left[v_{x}^{k}, v_{x}^{k}\right]\right]+\int \frac{v_{y y}^{k} v_{y y x}^{k}}{u^{0}}=\int\left(-f v_{x y}^{k}+g v_{x}^{k}\right) d y
$$

which is equivalent to

$$
\left[\left[v_{x}^{k}, v_{x}^{k}\right]\right]+\frac{1}{2} \frac{d}{d x} \int \frac{\left\{v_{y y}^{k}\right\}^{2}}{u^{0}}=\int\left(-f v_{x y}^{k}+g v_{x}^{k}-\frac{1}{2}\left\{\frac{1}{u^{0}}\right\}_{x}\left\{v_{y y}^{k}\right\}^{2}\right) d y .
$$


By the positivity estimate (1.16) and (1.17) with $u^{0}$, we note that $\left[\left[v_{x}^{k}, v_{x}^{k}\right]\right] \geq \theta_{0}\left\|v_{x y}^{k}\right\|_{L^{2}(0, N)}^{2}$. The standard Gronwall inequality then yields

$$
\sup _{x \in[0, L]} \int\left|v_{y y}^{k}\right|^{2}+\left\|v_{x y}^{k}\right\|_{L^{2}}^{2} \leq C\left(\left\|v_{y y}^{k}\right\|_{L^{2}(\{x=0\})}^{2}+\|f\|_{L^{2}}^{2}+\left\|\langle y\rangle^{3 / 2} g\right\|_{L^{2}}^{2}\right)
$$

in which we have used the inequality $\left\|g v_{x}^{k}\right\|_{L^{1}} \leq C\left\|v_{x y}^{k}\right\|_{L^{2}}\left\|\langle y\rangle^{3 / 2} g\right\|_{L^{2}}$. Taking limit as $k \rightarrow \infty$, we obtain the solution to (2.48) at once. This also proves the claim (2.49) when $j=n=0$.

Next, we shall derive high regularity estimates. We take $x$-derivative of (2.50) to get

$$
\left[\left[v_{x x}^{k}, e^{i}\right]\right]+\int \frac{v_{x y y}^{k} e_{y y}^{i}}{u^{0}}=-\int\left(\left\{\frac{u_{y y}^{0}}{u^{0}}\right\}_{x} v_{x}^{k} e^{i}+\left\{\frac{1}{u^{0}}\right\}_{x} v_{y y}^{k} e_{y y}^{i}\right)+\int\left(-f_{x} e_{y}^{i}+g_{x} e^{i}\right) d y .
$$

Recall now that $v_{x x}^{i}=\sum_{i=1}^{k} a_{x x}^{i} e^{i}$, which is a smooth function, since $a^{i}, e^{i}$ are both smooth. Using $v_{x x}^{i}$ as a test function, we then get

$$
\begin{aligned}
& {\left[\left[v_{x x}^{k}, v_{x x}^{k}\right]\right]+\frac{1}{2} \frac{d}{d x} \int \frac{\left\{v_{y y x}^{k}\right\}^{2}}{u^{0}} } \\
= & -\frac{1}{2} \int\left\{\frac{1}{u^{0}}\right\}_{x}\left\{v_{x y y}^{k}\right\}^{2}-\int\left(\left\{\frac{u_{y y}^{0}}{u^{0}}\right\}_{x} v_{x}^{k} v_{x x}^{k}+\left\{\frac{1}{u^{0}}\right\}_{x} v_{y y}^{k} v_{x x y y}^{k}\right)+\int\left(-f_{x} v_{x x y}^{k}+g_{x} v_{x x}^{k}\right) d y .
\end{aligned}
$$

The Gronwall inequality, together with (2.51), yields the claim (2.49) for the unweighted estimates, upon integrating by parts in $y$ the third term on the right. Almost identically, we may now insert the weight function $w(y)=\langle y\rangle^{2 n}$ and take inner products against $w(y) v_{x}^{k}$ and $w(y) v_{x x}^{k}$, respectively in the above energy estimates to obtain the weighted estimates as claimed. We avoid repeating the details.

Proof of Lemma 2.6. To apply the previous step, we first take care of the non-zero boundary conditions (2.44). Indeed, let us take $\chi(\cdot)$ to be a cutoff function near 0 with $\chi(0)=1$, and introduce

$$
\bar{v}=v_{p}(x, y)-y \chi(y) u_{e x}^{1}(x, 0) .
$$

Hence, $\bar{v}=\bar{v}_{y}=0$ at both $y=0$ and $y=N$. In addition, from (2.43), $\bar{v}$ solves

$$
\begin{aligned}
-\bar{v}_{x y y}+\frac{u_{y y}^{0}}{u^{0}} \bar{v}_{x}+\left(\frac{\bar{v}_{y y}}{u^{0}}\right)_{y y}= & G_{p x}-\left(\frac{u_{y y}^{0}}{u^{0}}\right)_{x} v_{p}+\left(u_{p y}\left(\frac{1}{u^{0}}\right)_{x}\right)_{y y}-(y \chi)_{y y} u_{e x x}^{1}(x, 0) \\
& +\frac{u_{y y}^{0}}{u^{0}} y \chi u_{e x x}^{1}(x, 0)+\left(\frac{(y \chi)_{y y}}{u^{0}}\right)_{y y} u_{e x}^{1}(x, 0) \\
= & : f_{y}+g,
\end{aligned}
$$


in which $G_{p}$ is defined as in (2.42). Explicitly, we have defined

$$
\begin{aligned}
f:= & u_{p y y}\left(\frac{1}{u^{0}}\right)_{x}+u_{p y}\left(\frac{1}{u^{0}}\right)_{x y}+\left(\frac{v_{p}^{0}+v_{e}^{1}}{u^{0}}\right) v_{p y y}+2 v_{p y y}\left(\frac{1}{u^{0}}\right)_{y}+\frac{v_{e x}^{1}}{u^{0}} u_{p y} \\
g:= & \left(\frac{1}{u^{0}}\right)_{x}\left(F_{p y}-u_{p} u_{x y}^{0}-\left[v_{p}^{0}+v_{e}^{1}\right] u_{p y y}-\sqrt{\varepsilon} v_{e z}^{1} u_{p y}\right)-\left(\frac{v_{e x}^{1}}{u^{0}}\right)_{y} u_{p y} \\
& +\frac{1}{u^{0}}\left(F_{p x y}-u_{p x} u_{x y}^{0}-u_{p} u_{x x y}^{0}-v_{p x}^{0} u_{p y y}-\sqrt{\varepsilon} v_{e x z}^{1} u_{p y}-\sqrt{\varepsilon} v_{e z}^{1} u_{p x y}\right) \\
& -\left(\frac{v_{p}^{0}+v_{e}^{1}}{u^{0}}\right)_{y} v_{p y y}-2 v_{p y y}\left(\frac{1}{u^{0}}\right)_{y y}-2 u_{p y y}\left(\frac{1}{u^{0}}\right)_{x y}-u_{p x y}\left(\frac{1}{u^{0}}\right)_{y y}-u_{p y}\left(\frac{1}{u^{0}}\right)_{x y y} \\
& -\left(\frac{u_{y y}^{0}}{u^{0}}\right)_{x} v_{p}-(y \chi)_{y y} u_{e x x}^{1}(x, 0)+\frac{u_{y y}^{0}}{u^{0}} y \chi u_{e x x}^{1}(x, 0)+\left(\frac{(y \chi)_{y y}}{u^{0}}\right)_{y y} u_{e x}^{1}(x, 0)
\end{aligned}
$$

with

$$
F_{p}=-u_{e z}^{0}\left[y u_{p x}^{0}+v_{p}^{0}\right]-y v_{e z}^{1} u_{p y}^{0}-u_{e}^{1} u_{p x}^{0}-u_{p}^{0} u_{e x}^{1} .
$$

Here, we note that the divergence-free condition is imposed: $u_{p x}=-v_{p y}=-\bar{v}_{y}+(y \chi)_{y} u_{e x}^{1}(x, 0)$. We construct the unique solution $\bar{v}$ to the above problem, and hence the solution $v_{p}$ to (2.43) via a contraction mapping theorem. We shall work with the norm:

$$
\left\||\bar{v} \|| \equiv \sup _{0 \leq x \leq L} \int\left|\bar{v}_{y y}\right|^{2}+\iint\left|\bar{v}_{x y}\right|^{2} d x d y .\right.
$$

Lemma 2.8 (or precisely, the estimate (2.51)) yields

$$
\|\bar{v}\| \| \leq C\left(\left\|\bar{v}_{y y}\right\|_{L^{2}(\{x=0\})}^{2}+\|f\|_{L^{2}}^{2}+\left\|\langle y\rangle^{3 / 2} g\right\|_{L^{2}}^{2}\right)
$$

with $(f, g)$ defined as in (2.52). Recall that $\bar{v}=v_{p}(x, y)+y \chi(y) v_{e z}^{1}(x, 0)$ with $v_{e}^{1}$ given on the boundary $x=0$. Hence, $\bar{v}_{y y}(0, y)$ can be estimates as follows, thanks to Lemma 2.7.

$$
\left\|v_{\text {pyy }}(0, \cdot)\right\|_{L^{2}\left(\mathbb{R}_{+}\right)} \leq C\left(u^{0}, v_{p}^{0},\left[u_{e}^{1}, v_{e}^{1}\right](0, \cdot)\right)\left(1+\left\|\bar{u}_{1}\right\|_{H^{4}\left(\mathbb{R}_{+}\right)}\right) .
$$

The uniform bound on $\left\|\bar{v}_{y y}\right\|_{L^{2}(\{x=0\})}^{2}$ follows.

Next, let us give bounds on $f, g$. For instance, $|\bar{v}| \leq y^{3 / 2}\left\|v_{y y}\right\|_{L^{2}\left(\mathbb{R}_{+}\right)},\left|\bar{v}_{y}\right| \leq y^{1 / 2}\left\|v_{y y}\right\|_{L^{2}\left(\mathbb{R}_{+}\right)}$, and thus

$$
\begin{aligned}
& \iint\langle y\rangle^{-n}|\bar{v}|^{2} \leq C \sup _{x}\left\|v_{y y}\right\|_{L^{2}\left(\mathbb{R}_{+}\right)}^{2} \iint\langle y\rangle^{-n+3} \leq C L\|\mid \bar{v}\| \|, \\
& \iint\langle y\rangle^{-n}\left|\bar{v}_{y}\right|^{2} \leq C \sup _{x}\left\|v_{y y}\right\|_{L^{2}\left(\mathbb{R}_{+}\right)}^{2} \iint\langle y\rangle^{-n+1} \leq C L\|\mid \bar{v}\| \|,
\end{aligned}
$$

for some large $n$. Such a spatial decay $\langle y\rangle^{-n}$ is produced by the rapid decay property of $u_{p}^{0}$. Similarly, we have

$$
\iint\langle y\rangle^{-n}\left|u_{p}\right|^{2} \leq C \iint\langle y\rangle^{-n}\left[\left|\bar{u}_{1}\right|^{2}+L \int_{0}^{L}\left|u_{p x}\right|^{2}\right] \leq C L\left(\left\|\bar{u}_{1}\right\|_{L^{2}\left(\mathbb{R}_{+}\right)}^{2}+\left\|u_{e x}^{1}(x, 0)\right\|_{L^{2}(0, L)}^{2}+\||\bar{v} \||),\right.
$$

using the fact that $u_{p x}=-v_{p y}=-\bar{v}_{y}+(y \chi)_{y} u_{e x}^{1}(x, 0)$. As for $u_{p y}$, we use $\left|u_{p y}\right| \leq\left|\bar{u}_{1 y}\right|+$ $\sqrt{L}\left\|u_{p x y}\right\|_{L^{2}(0, L)}$. Next, we bound $\langle y\rangle^{-n} u_{p y y}$, which by observation the decaying factor $\langle y\rangle^{-n}$ is 
always present, for large $n$, due to the decay property of $\left[u_{p}^{0}, v_{p}^{0}\right]$. To do so, we use (2.41) to estimate

$$
\iint\langle y\rangle^{-n}\left|u_{p y y}\right|^{2} \leq \iint\langle y\rangle^{-n}\left|u^{0} u_{p x}+u_{p} u_{x}^{0}+v_{p} u_{y}^{0}+\left[v_{p}^{0}+v_{e}^{1}\right] u_{p y}-F_{p}\right|^{2}
$$

which is again bounded by $C+C L \mid\|v\| \|$. Finally, we note that

$$
\begin{aligned}
\left\|u_{\text {exx }}^{1}(x, 0)\right\|_{L^{2}}^{2} & \leq \iint\left|v_{\text {exz }}^{1}(x, z) v_{\text {exzz }}^{1}(x, z)\right| \\
& \leq\left\|v_{\text {exz }}^{1}\right\|_{L^{q^{\prime}}}\left\|v_{\text {exzz }}^{1}\right\|_{L^{q}} \leq C(L, q) \varepsilon^{-1+1 / q}
\end{aligned}
$$

for arbitrary pair $\left(q, q^{\prime}\right)$ so that $1 / q+1 / q^{\prime}=1$; here, we take $q \rightarrow 1$.

Taking $L$ sufficiently small in the above estimates and in (2.53) yields a uniform bound on $\||\bar{v}\|\mid\|$ :

$$
\|\bar{v}\| \mid \leq C(L, \kappa) \varepsilon^{-\kappa}
$$

for arbitrarily small $\kappa>0$. Since the equation is linear in $\bar{v}$, this assures the existence of the unique solution to (2.52) and hence to (2.43). In addition, the above construction can be repeated to obtain a global solution in $x$ for any given $L$ for the existence of the Euler data. Finally, taking $N \rightarrow \infty$, we obtain the solution to (2.41) over $[0, \infty] \times \mathbb{R}_{+}$. The claimed weighted estimates follow similarly, using the rapid decay property of $u_{p}^{0}$.

In addition, the boundedness of $v_{p}$ follows by the calculation:

$$
\begin{aligned}
|v(x, y)|^{2} & \leq \int_{0}^{y}\left|v v_{y}\right| d y \leq \int_{0}^{\infty}\langle y\rangle^{-n}|v| d y+\int_{0}^{\infty}\langle y\rangle^{n}\left|v_{y}\right|^{2} d y \\
& \leq C \sup _{x}\left\|v_{y y}\right\|_{L^{2}\left(\mathbb{R}_{+}\right)}^{2}+\int_{0}^{\infty}\langle y\rangle^{n}\left|v_{y}(0, y)\right|^{2} d y+C \iint\langle y\rangle^{n}\left|v_{x y}\right|^{2} d x d y,
\end{aligned}
$$

which is bounded thanks to the previous bound on $\||v|\|$ and the uniform estimate of $v_{p}$ on the boundary $x=0$. Similarly, boundedness of $u_{p}$ follows from the definition

$$
u_{p}(x, y)=\bar{u}_{1}(y)+\int_{0}^{x} v_{p y} d x
$$

in which

$$
\int_{0}^{L}\left|v_{p y}\right|^{2} d x \leq \iint\langle y\rangle^{-n}\left|v_{p y}\right|^{2}+\iint\langle y\rangle^{n}\left|v_{p y y}\right|^{2}
$$

which is again bounded by $\|\mid v\| \|$.

To complete the proof of the lemma, we are now concerned with the higher regularity estimate. Again, applying Lemma 2.8 to the equation (2.52) yields

$$
\begin{aligned}
\sup _{0 \leq x \leq L} & \left\|\langle y\rangle^{n} v_{x y y}\right\|_{L^{2}\left(\mathbb{R}_{+}\right)}+\left\|\langle y\rangle^{n} v_{x x y}\right\|_{L^{2}} \\
& \leq C \sum_{k=0}^{1}\left(\left\|\langle y\rangle^{n} \partial_{x}^{k} v_{y y}\right\|_{L^{2}(\{x=0\})}+\left\|\langle y\rangle^{n} \partial_{x}^{k} f\right\|_{L^{2}}+\left\|\langle y\rangle^{n+\frac{3}{2}} \partial_{x}^{k} g\right\|_{L^{2}}\right) .
\end{aligned}
$$

Let us give bounds on the boundary term on $x=0$. Recall that $v=v_{p}(x, y)-y \chi(y) u_{e x}^{1}(x, 0)$. Lemma 2.7 gives

$$
\left\|\langle y\rangle^{n} v_{p x y y}(0, \cdot)\right\|_{L^{2}\left(\mathbb{R}_{+}\right)} \leq C_{0}\left(1+\left\|\langle y\rangle^{-m} u_{e x x}^{1}(0, \sqrt{\varepsilon} \cdot)\right\|_{H^{1}\left(\mathbb{R}_{+}\right)}\right) .
$$


Using the inequality $|L f(0)| \leq \int_{0}^{L}\left|[(L-x) f(x)]_{x}\right| d x \leq \sqrt{L}\|f\|_{L^{2}}+L^{3 / 2}\left\|f_{x}\right\|_{L^{2}}$, we have

$$
\begin{aligned}
\left\|u_{\text {exx }}^{1}(0, \sqrt{\varepsilon} \cdot)\right\|_{L^{2}\left(\mathbb{R}_{+}\right)} & \leq C \varepsilon^{-1 / 4}\left\|v_{\text {exz }}^{1}(0, \cdot)\right\|_{L^{2}\left(\mathbb{R}_{+}\right)} \\
& \leq C \varepsilon^{-1 / 4} L^{-1 / 2}\left(\left\|v_{\text {exz }}^{1}(\cdot)\right\|_{L^{2}}+\left\|v_{\text {exz }}^{1}(\cdot)\right\|_{L^{2}}^{1 / 2}\left\|v_{\text {exxz }}^{1}(\cdot)\right\|_{L^{2}}^{1 / 2}\right) \\
& \leq C \varepsilon^{-1 / 2} L^{-1 / 2}
\end{aligned}
$$

and

$$
\begin{aligned}
\left\|u_{\text {exxy }}^{1}(0, \sqrt{\varepsilon} \cdot)\right\|_{L^{2}\left(\mathbb{R}_{+}\right)} & \leq C \varepsilon^{1 / 4}\left\|v_{\text {exzz }}^{1}(0, \cdot)\right\|_{L^{2}\left(\mathbb{R}_{+}\right)} \\
& \leq C \varepsilon^{1 / 4} L^{-1 / 2}\left(\left\|v_{\text {exzz }}^{1}(\cdot)\right\|_{L^{2}}+\left\|v_{\text {exzz }}^{1}(\cdot)\right\|_{L^{2}}^{1 / 2}\left\|v_{\text {exxzz }}^{1}(\cdot)\right\|_{L^{2}}^{1 / 2}\right) \\
& \leq C \varepsilon^{-3 / 4} L^{-1 / 2}
\end{aligned}
$$

in which the estimates on $v_{e}^{1}$ from Lemma 2.5 were used. Also, we have

$$
\begin{aligned}
\left|u_{\text {exx }}^{1}(0,0)\right|^{2} & \leq\left\|u_{\text {exx }}^{1}(0, \cdot)\right\|_{L^{2}\left(\mathbb{R}_{+}\right)}\left\|v_{\text {exzz }}^{1}(0, \cdot)\right\|_{L^{2}\left(\mathbb{R}_{+}\right)} \\
& \leq C L^{-1 / 2}\left\|u_{\text {exx }}^{1}(0, \cdot)\right\|_{L^{2}\left(\mathbb{R}_{+}\right)}\left(\left\|v_{\text {exzz }}^{1}(\cdot)\right\|_{L^{2}}+\left\|v_{\text {exzz }}^{1}(\cdot)\right\|_{L^{2}}^{1 / 2}\left\|v_{\text {exzzz }}^{1}(\cdot)\right\|_{L^{2}}^{1 / 2}\right) \\
& \leq C \varepsilon^{-3 / 2} L^{-1}
\end{aligned}
$$

This proves that

$$
\left\|\langle y\rangle^{n} v_{\text {pxyy }}(0, \cdot)\right\|_{L^{2}\left(\mathbb{R}_{+}\right)} \leq C \varepsilon^{-3 / 4} L^{-1 / 2},
$$

uniformly in small $\varepsilon$ and $L$. Next, estimates for $f$ and $g$ are treated similarly as done above. In particular, we note

$$
\begin{aligned}
\left\|u_{\text {exxx }}^{1}(x, 0)\right\|_{L^{2}}^{2} & \leq \iint\left|v_{\text {exxz }}^{1}(x, z) v_{\text {exxzz }}^{1}(x, z)\right| \\
& \leq\left\|v_{\text {exx }}^{1}\right\|_{L^{2}}\left\|v_{\text {exx } z z}^{1}\right\|_{L^{2}} \leq C(L) \varepsilon^{-2},
\end{aligned}
$$

which together with the previous estimates yield the estimate (2.46).

This completes the proof of the lemma.

\subsubsection{Cut-off Prandtl layers}

Finally, we are ready to introduce the Prandtl layers that we shall use in the boundary layer expansion. Let us define a cutoff function $\chi(\cdot)$ with support in $[0,1]$, and let $\left[u_{p}, v_{p}\right]$ be constructed as in the previous section. We introduce

$$
u_{p}^{1}=\chi(\sqrt{\varepsilon} y) u_{p}+\sqrt{\varepsilon} \chi^{\prime}(\sqrt{\varepsilon} y) \int_{0}^{y} u_{p}(x, s) d s, \quad v_{p}^{1}=\chi(\sqrt{\varepsilon} y) v_{p} .
$$

Clearly, $\left[u_{p}^{1}, v_{p}^{1}\right]$ is a divergence-free vector field. By the estimates from Lemma 2.6 on $\left[u_{p}, v_{p}\right]$, we get

$$
\left|\sqrt{\varepsilon} \chi^{\prime}(\sqrt{\varepsilon} y) \int_{0}^{y} u_{p}(x, s) d s\right| \leq \sqrt{\varepsilon} y\left|\chi^{\prime}(\sqrt{\varepsilon} y)\right|\left\|u_{p}\right\|_{\infty} \leq C(L, \kappa) \varepsilon^{-\kappa} .
$$

Hence, Lemma 2.6 now reads we have

$$
\begin{aligned}
\left\|\left[u_{p}^{1}, v_{p}^{1}\right]\right\|_{\infty} & +\sup _{0 \leq x \leq L}\left\|\langle y\rangle^{n} v_{p y y}^{1}\right\|_{L^{2}\left(\mathbb{R}_{+}\right)}+\left\|\langle y\rangle^{n} v_{p x y}^{1}\right\|_{L^{2}} \leq C(L, \kappa) \varepsilon^{-\kappa} \\
& \sup _{0 \leq x \leq L}\left\|\langle y\rangle^{n} v_{p x y y}^{1}\right\|_{L^{2}\left(\mathbb{R}_{+}\right)}+\left\|\langle y\rangle^{n} v_{p x x y}^{1}\right\|_{L^{2}} \leq C(L) \varepsilon^{-1}
\end{aligned}
$$


uniformly in small $\varepsilon, L$, and for arbitrarily small $\kappa$. In addition, thanks to the cut-off function, we also have

$$
\begin{aligned}
\left\|v_{p x}^{1}\right\|_{L^{2}}^{2} & \leq \iint_{\{\sqrt{\varepsilon} y \leq 1\}}\left|v_{p x}^{1}\right|^{2} d x d y \lesssim \varepsilon^{-1 / 2} \iint\langle y\rangle^{n}\left|v_{p x y}^{1}\right|^{2} d x d y \leq C(L, \kappa) \varepsilon^{-1 / 2-2 \kappa} \\
\left\|v_{p y}^{1}\right\|_{L^{2}}^{2} & \leq \iint_{\{\sqrt{\varepsilon} y \leq 1\}}\left|v_{p y}^{1}\right|^{2} d x d y \lesssim \varepsilon^{-1 / 2} \iint\langle y\rangle^{n}\left|v_{p y y}^{1}\right|^{2} d x d y \leq C(L, \kappa) \varepsilon^{-1 / 2-2 \kappa} \\
\left\|v_{p x x}^{1}\right\|_{L^{2}}^{2} & \leq \iint_{\{\sqrt{\varepsilon} y \leq 1\}}\left|v_{p x x}^{1}\right|^{2} d x d y \lesssim \varepsilon^{-1 / 2} \iint\langle y\rangle^{n}\left|v_{p x x y}^{1}\right|^{2} d x d y \leq C(L) \varepsilon^{-5 / 2} .
\end{aligned}
$$

We now plug $\left[u_{p}^{1}, v_{p}^{1}\right]$ into (2.41), or equivalently, (2.18). It does not solve it completely, yielding a new error due to the above cut-off:

$$
\begin{aligned}
R_{p}^{u, 1}:= & \left(u^{0} \partial_{x}+u_{x}^{0}+\left[v_{p}^{0}+v_{e}^{1}\right] \partial_{y}-\partial_{y}^{2}\right)\left(\sqrt{\varepsilon} \chi^{\prime}(\sqrt{\varepsilon} y) \int_{0}^{y} u_{p} d s\right)-2 \sqrt{\varepsilon} \chi^{\prime}(\sqrt{\varepsilon} y)\left[v_{p}^{0}+v_{e}^{1}\right] u_{p y} \\
& +u_{p}\left[v_{p}^{0}+v_{e}^{1}\right]\left(\sqrt{\varepsilon} \chi^{\prime}(\sqrt{\varepsilon} y)-\varepsilon \chi^{\prime \prime}(\sqrt{\varepsilon} y)\right) \\
& +(1-\chi(\sqrt{\varepsilon} y))\left(u_{e z}^{0}\left[y u_{p x}^{0}+v_{p}^{0}\right]+y v_{e z}^{1} u_{p y}^{0}+u_{e}^{1} u_{p x}^{0}+u_{p}^{0} u_{e x}^{1}\right)
\end{aligned}
$$

which contributes into $R^{u, 1}$ in (2.37) (and hence $R_{\mathrm{app}}^{u}$ ). Let us give an estimate on this error term. Recall that $u_{p}^{0}$ is rapidly decaying at infinity, and so $u_{x}^{0}=u_{p x}^{0}$ also decays rapidly. Hence, the integral $u_{x}^{0} \int_{0}^{y} u_{p} d s$ is uniformly bounded by $\varepsilon^{-\kappa}$. Together with boundedness of the constructed Euler and Prandtl layers, we have $L^{2}$ norm of the first three big terms involving $u_{p}$ in $R_{p}^{u, 1}$ is bounded by

$$
C \varepsilon^{-\kappa} \sqrt{\varepsilon}\|\chi(\sqrt{\varepsilon} \cdot)\|_{L^{2}} \leq C \varepsilon^{1 / 4-\kappa} .
$$

Now, as for the term

$$
(1-\chi(\sqrt{\varepsilon} y))\left(u_{e z}^{0}\left[y u_{p x}^{0}+v_{p}^{0}\right]+y v_{e z}^{1} u_{p y}^{0}+u_{e}^{1} u_{p x}^{0}+u_{p}^{0} u_{e x}^{1}\right)
$$

we note that the Prandtl layers $\left[u_{p}^{0}, v_{p}^{0}\right]$ is rapidly decaying in $y \rightarrow \infty$, this error term is thus bounded by $C e^{-y}$, which is of order $\varepsilon^{n}$ in the region where $\sqrt{\varepsilon} y \geq 1$, for arbitrary large $n \geq 0$. Combining with the above estimates proves that

$$
\left\|R_{p}^{u, 1}\right\|_{L^{2}} \lesssim C(L, \kappa) \varepsilon^{1 / 4-\kappa} .
$$

Finally, by a view of definition of $p_{p}^{2}$ from (2.22), we estimate $p_{p x}^{2}$ :

$$
\begin{aligned}
p_{p x}^{2} & =\int_{y}^{\infty} \partial_{x}\left[\left[u_{e}^{0}+u_{p}^{0}\right] v_{p x}^{0}+u_{p}^{0} v_{e x}^{1}+\left[v_{p}^{0}+v_{e}^{1}\right] v_{p y}^{0}-v_{p y y}^{0}\right](x, \theta) d \theta \\
& =\int_{y}^{\infty}\left[\left[u_{e}^{0}+u_{p}^{0}\right] v_{p x x}^{0}+u_{p}^{0} v_{e x x}^{1}+\left[v_{p}^{0}+v_{e}^{1}\right] v_{p x y}^{0}-v_{p x y y}^{0}\right](x, \theta) d \theta
\end{aligned}
$$

the last identity was obtained with a use of the divergence-free condition on the vector field $\left[u_{p}^{0}, v_{p}^{0}\right]$. We estimate each term on the right. Thanks to the boundedness of $u_{e}^{0},\left[u_{p}^{0}, v_{p}^{0}\right]$ and $v_{e}^{1}$, and the 
rapid decay property of the Prandtl layers $\left[u_{p}^{0}, v_{p}^{0}\right]$, we note that

$$
\begin{gathered}
\int_{y}^{\infty}\left[u_{e}^{0}+u_{p}^{0}\right] v_{p x x}^{0} \leq C\langle y\rangle^{-n}\left\|u_{e}^{0}+u_{p}^{0}\right\|_{L^{\infty}}\left\|\langle y\rangle^{n} v_{p x x}^{0}\right\|_{L^{2}\left(\mathbb{R}_{+}\right)} \\
\int_{y}^{\infty} u_{p}^{0} v_{e x x}^{1} \leq C\langle y\rangle^{-n}\left\|\langle y\rangle^{n} u_{p}^{0}\right\|_{L^{2}\left(\mathbb{R}_{+}\right)}\left\|v_{e x x}^{1}(x, \sqrt{\varepsilon} \cdot)\right\|_{L^{2}\left(\mathbb{R}_{+}\right)} \\
\int_{y}^{\infty}\left[v_{p}^{0}+v_{e}^{1}\right] v_{p x y}^{0} \leq C\langle y\rangle^{-n}\left\|v_{p}^{0}+v_{e}^{1}\right\|_{L^{\infty}}\left\|\langle y\rangle^{n} v_{p x y}^{0}\right\|_{L^{2}\left(\mathbb{R}_{+}\right)} \\
\int_{y}^{\infty} v_{p x y y}^{0} \leq C\langle y\rangle^{-n}\left\|\langle y\rangle^{n} v_{p x y y}^{0}\right\|_{L^{2}\left(\mathbb{R}_{+}\right)} .
\end{gathered}
$$

Hence, taking $n \geq 2$ and using the known bounds on the profile solutions, we immediately get

$$
\left\|p_{p x}^{2}\right\|_{L^{2}} \lesssim \varepsilon^{-1 / 4}
$$

\subsection{Proof of Proposition 2.1}

Having constructed the Euler and Prandtl layers, we now calculate the remaining errors in $R_{\text {app }}^{u}$ and $R_{\text {app }}^{v}$ from (2.2a) and (2.2b), respectively, and hence complete the proof of Proposition 2.1, To do so, collecting errors from $R^{u, 0}$ in (2.5),$R^{u, 1}$ in (2.38), the new error $R_{p}^{u, 1}$ in (2.58), and the remaining $\varepsilon$-order terms in $R_{\mathrm{app}}^{u}$, we get

$$
\begin{aligned}
R_{\mathrm{app}}^{u}=E_{0}-\varepsilon u_{e z z}^{0}+\sqrt{\varepsilon} R^{u, 1}+\sqrt{\varepsilon} R_{p}^{u, 1}+\varepsilon\left[\left[u_{e}^{1}+u_{p}^{1}\right] \partial_{x}+v_{p}^{1} \partial_{y}\right]\left[u_{e}^{1}+u_{p}^{1}\right] \\
+\varepsilon p_{p x}^{2}-\varepsilon \partial_{x}^{2}\left\{u_{p}^{0}+\sqrt{\varepsilon}\left[u_{e}^{1}+u_{p}^{1}\right]\right\}
\end{aligned}
$$

in which reading the estimates (2.38), (2.40), (2.58), (2.59) and using the fact that $u_{e z z}^{0}$ are bounded in $L^{2}$ in the original coordinates, we immediately have

$$
\left\|E_{0}-\varepsilon u_{e z z}^{0}+\sqrt{\varepsilon} R^{u, 1}+\sqrt{\varepsilon} R_{p}^{u, 1}+\varepsilon p_{p x}^{2}\right\|_{L^{2}} \leq C(L, \kappa) \varepsilon^{3 / 4-\kappa} .
$$

Similarly, using boundedness of $u_{e}^{1}, u_{p}^{1}, v_{p}^{1}$ and the $L^{2}$ bound on the derivatives of $u_{p}^{0}, u_{e}^{1}, u_{p}^{1}$ and keeping in mind that the Euler flows are evaluated at $(x, \sqrt{\varepsilon} y)$, we get

$$
\begin{aligned}
\varepsilon\left\|\left[u_{e}^{1}+u_{p}^{1}\right] \partial_{x}\left[u_{e}^{1}+u_{p}^{1}\right]\right\|_{L^{2}} & \leq \varepsilon\left[\left\|u_{e}^{1}\right\|_{L^{\infty}}+\left\|u_{p}^{1}\right\|_{L^{\infty}}\right]\left[\left\|u_{e x}^{1}\right\|_{L^{2}}+\left\|u_{p x}^{1}\right\|_{L^{2}}\right] \leq C(L, \kappa) \varepsilon^{3 / 4-\kappa} \\
\varepsilon\left\|v_{p}^{1} \partial_{y}\left[u_{e}^{1}+u_{p}^{1}\right]\right\|_{L^{2}} & \leq \varepsilon\left\|v_{p}^{1}\right\|_{L^{\infty}}\left[\left\|\sqrt{\varepsilon} u_{e z}^{1}\right\|_{L^{2}}+\left\|u_{p y}^{1}\right\|_{L^{2}}\right] \leq C(L, \kappa) \varepsilon^{1-\kappa} \\
\varepsilon\left\|\partial_{x}^{2}\left\{u_{p}^{0}+\sqrt{\varepsilon}\left[u_{e}^{1}+u_{p}^{1}\right]\right\}\right\|_{L^{2}} & \leq \varepsilon\left\|u_{p x x}^{0}\right\|_{L^{2}}+\varepsilon^{3 / 2}\left[\left\|u_{e x x}^{1}\right\|_{L^{2}}+\left\|u_{p x x}^{1}\right\|_{L^{2}}\right] \leq C \varepsilon,
\end{aligned}
$$

in which $\kappa$ is arbitrarily small constant (we choose $\frac{\kappa}{2}$ in Lemma 2.6). This proves that

$$
\left\|R_{\text {app }}^{u}\right\|_{L^{2}} \leq C(L, \kappa) \varepsilon^{3 / 4-\kappa} .
$$

Next, we calculate the error $R_{\text {app }}^{v}$ from $(2.2 \mathrm{~b})$. Simply collecting the remaining terms in $R^{v, 0}$ (see (2.23) $)$ and all terms with a factor $\sqrt{\varepsilon}$ or small, we get

$$
\begin{aligned}
R_{\mathrm{app}}^{v}= & R^{v, 0}+\sqrt{\varepsilon}\left[\left\{u_{e}^{0}+u_{p}^{0}+\sqrt{\varepsilon}\left[u_{e}^{1}+u_{p}^{1}\right]\right\} \partial_{x}+\left\{v_{p}^{0}+v_{e}^{1}+\sqrt{\varepsilon} v_{p}^{1}\right\} \partial_{y}\right] v_{p}^{1} \\
& +\sqrt{\varepsilon}\left[\left[u_{e}^{1}+u_{p}^{1}\right] \partial_{x}+v_{p}^{1} \partial_{y}\right]\left[v_{p}^{0}+v_{e}^{1}\right]-\sqrt{\varepsilon} v_{\text {pyy }}^{1}-\varepsilon \partial_{x}^{2}\left\{v_{p}^{0}+v_{e}^{1}+\sqrt{\varepsilon} v_{p}^{1}\right\}
\end{aligned}
$$


By (2.39), we have $\left\|R^{v, 0}\right\|_{L^{2}} \leq C \varepsilon^{1 / 4}$. We now estimate the remaining terms one by one in $R_{\text {app }}^{v}$. Similarly as above, using the boundedness of all profile solutions, we get

$$
\begin{aligned}
\sqrt{\varepsilon}\left\|\left[\left\{u_{e}^{0}+u_{p}^{0}+\sqrt{\varepsilon}\left[u_{e}^{1}+u_{p}^{1}\right]\right\} \partial_{x}+\left\{v_{p}^{0}+v_{e}^{1}+\sqrt{\varepsilon} v_{p}^{1}\right\} \partial_{y}\right] v_{p}^{1}\right\|_{L^{2}} & \leq C \sqrt{\varepsilon}\left\|\left[u_{e}^{0}, u_{p}^{0}, v_{p}^{0}, u_{e}^{1}, v_{e}^{1}\right]\right\|_{L^{\infty}}\left\|\nabla v_{p}^{1}\right\|_{L^{2}} \\
& \leq C(L, \kappa) \varepsilon^{1 / 4-\kappa}
\end{aligned}
$$

upon recalling the bound $\left\|v_{p x}^{1}\right\|_{L^{2}} \leq C(L) \varepsilon^{-1 / 4-\kappa}$. Next, we have

$$
\begin{aligned}
\sqrt{\varepsilon}\left\|\left[\left[u_{e}^{1}+u_{p}^{1}\right] \partial_{x}+v_{p}^{1} \partial_{y}\right]\left[v_{p}^{0}+v_{e}^{1}\right]\right\|_{L^{2}} & \leq C \sqrt{\varepsilon}\left\|\left[u_{e}^{1}, u_{p}^{1}, v_{p}^{1}\right]\right\|_{L^{\infty}}\left(\left\|v_{p x}^{0}+v_{e x}^{1}\right\|_{L^{2}}+\left\|v_{p y}^{0}+\sqrt{\varepsilon} v_{e z}^{1}\right\|_{L^{2}}\right) \\
& \leq C(L, \kappa) \varepsilon^{1 / 4-\kappa},
\end{aligned}
$$

with noting that $\left\|v_{e x}^{1}(\cdot, \sqrt{\varepsilon} \cdot)\right\|_{L^{2}} \leq C \varepsilon^{-1 / 4}$. Finally, it is clear that

$$
\left\|\sqrt{\varepsilon} v_{p y y}^{1}-\varepsilon \partial_{x}^{2}\left\{v_{p}^{0}+v_{e}^{1}+\sqrt{\varepsilon} v_{p}^{1}\right\}\right\|_{L^{2}} \leq C(L) \varepsilon^{1 / 4-\kappa},
$$

since $\left\|\left[v_{p y y}^{1}, v_{p x x}^{0}\right]\right\|_{L^{2}}$ is uniformly bounded by $C(L, \kappa) \varepsilon^{-\kappa},\left\|v_{e x x}^{1}\right\|_{L^{2}} \leq C \varepsilon^{-1 / 4}$, and $\left\|v_{p x x}^{1}\right\|_{L^{2}} \leq$ $C(L) \varepsilon^{-5 / 4}$, as summarized in Section 2.4.2. Putting these together into $R_{\text {app }}^{v}$ and using the fact that $\varepsilon \ll L$, we have obtained

$$
\left\|R_{\mathrm{app}}^{v}\right\|_{L^{2}} \leq C(L, \kappa) \varepsilon^{1 / 4-\kappa} .
$$

This completes the proof of Proposition 2.1.

\section{$3 \quad$ Linear stability estimates}

This section is devoted to prove the following crucial linear stability estimates for the linearized equations around the constructed approximate solutions $\left[u_{\text {app }}, v_{\text {app }}\right]$. Recall (1.15).

Proposition 3.1. Let $\left[u_{s}, v_{s}\right]$ be the approximate solution defined as in (1.15). For any given $f, g$ in $L^{2}$, there exists a positive number $L$ so that the following linear problem

$$
\begin{aligned}
& u_{s} u_{x}+u u_{s x}+v_{s} u_{y}+v u_{s y}+p_{x}-\Delta_{\varepsilon} u=f \\
& u_{s} v_{x}+u v_{s x}+v_{s} v_{y}+v v_{s y}+\frac{p_{y}}{\varepsilon}-\Delta_{\varepsilon} v=g
\end{aligned}
$$

together with the divergence-free condition $u_{x}+v_{y}=0$ and boundary conditions

$$
\begin{array}{rlrl}
{[u, v]_{y=0}=0 \quad(\text { no-slip }),} & {[u, v]_{x=0}=0 \quad \text { (Dirichlet) },} \\
p-2 \varepsilon u_{x}=0, & u_{y}+\varepsilon v_{x}=0 & \text { at } x=L \text { (Neumann or stress-free) } .
\end{array}
$$

has a unique solution $[u, v, p]$ on $[0, L] \times \mathbb{R}_{+}$. Furthermore, there holds

$$
\left\|\nabla_{\varepsilon} u\right\|_{L^{2}}+\left\|\nabla_{\varepsilon} v\right\|_{L^{2}} \lesssim\|f\|_{L^{2}}+\sqrt{\varepsilon}\|g\|_{L^{2}}
$$

The proof of Proposition 3.1 consists of several steps. First, we construct the solution in the artificial cut-off domain:

$$
\Omega_{N}:=\{0 \leq x \leq L, \quad 0 \leq y \leq N\}
$$


with the no-slip boundary conditions $[u, v]=0$ prescribed at $y=N$. We shall apply the standard Schaefer's fixed theorem (see, for instance, [4, Section 9.2.2]) for the space $X=\left\{\|[u, v]\|_{H^{1}\left(\Omega_{N}\right)} \leq\right.$ $C$ \}, for some fixed constant $C>0$. Without loss of generality, we may assume the source terms $f$ and $g$ are smooth. Then the standard regularity theory for the Stokes problem yields $H^{3}$ regularity for $[u, v]$, except at the four corners $[0,0],[0, N],[L, 0]$ and $[L, N]$. In addition, by [13, 14, 2], we know that $[u, v] \in H^{3 / 2+}$ and $p \in H^{1 / 2+}$ including the four corners, and hence, the $H^{1}$ norm of $[u, v]$ makes sense. Indeed by the trace theorem, we have

$$
\|\nabla[u, v]\|_{L^{2+}(\Gamma)}+\|p\|_{L^{2+}(\Gamma)} \lesssim\|[u, v]\|_{H^{3 / 2+}}+\|p\|_{H^{1 / 2+}},
$$

for any finite piece-wise $C^{1}$ curve $\Gamma$. We now take $\Gamma=\Gamma_{\delta}$ to be the curve of intersection of $\Omega_{N}$ and the circle of radius $\delta$ and centered at the four corners, respectively. Clearly,

$$
\int_{\Gamma_{\delta}}|\nabla u|^{2}+|\nabla v|^{2} \lesssim o(1)\left[|\nabla u|_{L^{2+}\left(\Gamma_{\delta}\right)}^{2}+|\nabla v|_{L^{2}\left(\Gamma_{\delta}\right)}^{2}\right] \lesssim o(1)\left[\|[u, v]\|_{H^{3 / 2+}}+\|p\|_{H^{1 / 2+}}\right]
$$

where $o(1) \rightarrow 0$ as $\delta \rightarrow 0$. This justifies the meaning of $H^{1}$ norm of the solution $[u, v]$ in the presence of corners.

We shall now derive uniform a priori estimates for (3.1)-(3.3). Taking the limit $N \rightarrow \infty$ yields the uniform a priori bound (3.4). The existence of the solution and hence the Proposition 3.1 would then follow from a direct application of the Schaefer's fixed point theorem; see [4, Theorem 4, p. 504] and Section [3.3, below. As will be seen shortly, the positivity estimate (1.16) plays a crucial role.

\subsection{Energy estimates}

Lemma 3.2. Let $[u, v]$ be the solution to the problem (3.1)-(3.3). Assume that $\varepsilon \ll L$. There holds

$$
\left\|\nabla_{\varepsilon} u\right\|_{2}^{2}+\int_{x=L} u_{s}\left(u^{2}+\varepsilon v^{2}\right) \lesssim L\left\|\nabla_{\varepsilon} v\right\|_{L^{2}}^{2}+\|f\|_{L^{2}}^{2}+\varepsilon\|g\|_{L^{2}}^{2} .
$$

Proof. We multiply (3.1) with $u$ and (3.2) with $\varepsilon v$ (or equivalently, take $[u, v]$ as the test function in the weak formulation) get

$$
\begin{aligned}
& \iint\left[u_{s} u_{x}+u_{s x} u+v_{s} u_{y}+v u_{s y}+p_{x}-\Delta_{\varepsilon} u\right] u \\
& +\iint \varepsilon\left[u_{s} v_{x}+u_{s x} v+v_{s} v_{y}+v v_{s y}+p_{y}-\varepsilon \Delta_{\varepsilon} v\right] v \\
= & \iint u f+\iint \varepsilon v g .
\end{aligned}
$$

By writing $\Delta_{\varepsilon} u=2 \varepsilon u_{x x}+\left(u_{y}+\varepsilon v_{x}\right)_{y}, \Delta_{\varepsilon} v=\left(u_{y}+\varepsilon v_{x}\right)_{x}+2 v_{y y}$ and performing the integration 
by parts multiple times, the left-hand side of the above is reduced to

$$
\begin{aligned}
& \iint\left\{u_{s} u_{x}+u_{s x} u+v_{s} u_{y}+v u_{s y}+p_{x}-\Delta_{\varepsilon} u\right\} u \\
& +\iint \varepsilon\left[u_{s} v_{x}+u_{s x} v+v_{s} v_{y}+v v_{s y}+p_{y}-\varepsilon \Delta_{\varepsilon} v\right] v \\
= & -\iint u_{s x} \frac{u^{2}+\varepsilon v^{2}}{2}+\left.u_{s} \frac{u^{2}+\varepsilon v^{2}}{2}\right|_{x=0} ^{x=L}+\iint u_{s x}\left[u^{2}+\varepsilon v^{2}\right]-\iint v_{s y} \frac{u^{2}+\varepsilon v^{2}}{2} \\
& +\iint\left[v u_{s y} u+\varepsilon v_{s y} v^{2}\right]+\left.\int p u\right|_{x=0} ^{x=L} \\
& -\left.2 \varepsilon \int u_{x} u\right|_{x=0} ^{x=L}+2 \varepsilon \iint u_{x}^{2}+\iint\left(u_{y}+\varepsilon v_{x}\right) u_{y} \\
& -\left.\varepsilon \int\left(u_{y}+\varepsilon v_{x}\right) v\right|_{x=0} ^{x=L}+\iint \varepsilon\left(u_{y}+\varepsilon v_{x}\right) v_{x}+2 \iint \varepsilon v_{y}^{2} .
\end{aligned}
$$

By using the boundary conditions $p=2 \varepsilon u_{x}$ at $x=L$ and $[u, v]=0$ at $x=0$, and the divergence-free condition $u_{s x}+v_{s y}=0$, the energy estimate now becomes

$$
\begin{aligned}
& \int_{x=L} u_{s} \frac{u^{2}+\varepsilon v^{2}}{2}+\iint\left[2 \varepsilon u_{x}^{2}+\left(u_{y}+\varepsilon v_{x}\right)^{2}+2 \varepsilon v_{y}^{2}\right] \\
= & -\iint u_{s x}\left[u^{2}+\varepsilon v^{2}\right]-\iint\left[v u_{s y} u+\varepsilon v_{s y} v^{2}\right]+\iint u f+\varepsilon \iint v g .
\end{aligned}
$$

Here, we note that

$$
\begin{aligned}
\iint\left[2 \varepsilon u_{x}^{2}+\left(u_{y}+\varepsilon v_{x}\right)^{2}+2 \varepsilon v_{y}^{2}\right] & =\iint\left[2 \varepsilon u_{x}^{2}+u_{y}^{2}+\varepsilon^{2} v_{x}+2 \varepsilon v_{y}^{2}\right]+2 \varepsilon \iint u_{y} v_{x} \\
& \geq \frac{1}{2}\left\|\nabla_{\varepsilon} u\right\|_{L^{2}}^{2}-2 \varepsilon\left\|\nabla_{\varepsilon} v\right\|_{L^{2}}^{2},
\end{aligned}
$$

in which we have used the Young inequality, giving the estimate $2 \varepsilon \iint u_{y} v_{x} \leq \frac{1}{2}\left\|u_{y}\right\|_{L^{2}}^{2}+2 \varepsilon\left\|\nabla_{\varepsilon} v\right\|_{L^{2}}^{2}$. In addition, since $[u, v]=0$ at $x=0$, we have the embedding inequalities $\|u\|_{L^{2}} \leq L\left\|u_{x}\right\|_{L^{2}}=$ $L\left\|v_{y}\right\|_{L^{2}},\|v\|_{L^{2}} \leq L\left\|v_{x}\right\|_{L^{2}}$. Hence,

$$
\begin{aligned}
\iint u f+\varepsilon \iint v g & \leq\|u\|_{L^{2}}\|f\|_{L^{2}}+\varepsilon\|v\|_{L^{2}}\|g\|_{L^{2}} \\
& \leq L^{2}\left\{\left\|v_{y}\right\|_{L^{2}}^{2}+\varepsilon\left\|v_{x}\right\|^{2}\right\}+\|f\|_{L^{2}}^{2}+\varepsilon\|g\|_{L^{2}}^{2} .
\end{aligned}
$$

Similarly, since $v=0$ at $y=0$, we can estimate $v=\int_{0}^{y} v_{y} \leq \sqrt{y}\left\{\int_{0}^{y} v_{y}^{2}\right\}^{1 / 2}$. Hence,

$$
\begin{aligned}
& -\iint u_{s x}\left[u^{2}+\varepsilon v^{2}\right]-\iint\left[v \partial_{y} u_{s} u+\varepsilon \partial_{y} v_{s} v^{2}\right] \\
\leq & L^{2} \iint\left|u_{s x}\right| u_{x}^{2}+\varepsilon \iint\left|y u_{s x}\right|\left\{\int_{0}^{y} v_{y}^{2}\right\}+\iint\left\{\int_{0}^{x}\left|u_{x}^{2} d x\right|\right\}^{1 / 2} \sqrt{y} \partial_{y} u_{s}\left\{\int_{0}^{y}\left|v_{y}^{2} d x\right|\right\}^{1 / 2} \\
& +\varepsilon \iint y \partial_{y} v_{s}\left\{\int_{0}^{y} v_{y}^{2} d y\right\} \\
\leq & \left\{L^{2} \sup \left|\partial_{x} u_{s}\right|+\varepsilon \sup _{x} \int\left|y \partial_{y} v_{s}\right| d y+L \sup _{x} \sqrt{\int y\left\{\partial_{y} u_{s}\right\}^{2} d y}\right\}\left\|v_{y}\right\|_{L^{2}}^{2} .
\end{aligned}
$$


This proves the claimed inequality in the lemma, with

$$
C\left(\varepsilon, L, u_{s}, v_{s}\right):=\left\{L^{2} \sup \left|u_{s x}\right|+\varepsilon \sup _{x} \int\left|y v_{s y}\right| d y+L \sup _{x} \sqrt{\int y\left\{u_{s y}\right\}^{2}} d y+L^{2}+2 \varepsilon\right\} .
$$

It remains to give the bound on the constant $C\left(\varepsilon, L, u_{s}, v_{s}\right)$. We recall that $u_{s}=u_{e}^{0}+u_{p}^{0}+\sqrt{\varepsilon} u_{e}^{1}$ and $v_{s}=v_{p}^{0}+v_{e}^{1}$. Keeping in mind that the zeroth-order Prandtl layers $\left[u_{p}^{0}, v_{p}^{0}\right]$ are smooth with arbitrarily high Sobolev regularity and rapidly decaying in $y$. Hence,

$$
\left\|u_{s x}\right\|_{\infty} \leq\left\|u_{p x}^{0}\right\|_{\infty}+\sqrt{\varepsilon}\left\|u_{e x}^{1}\right\|_{\infty} \leq C\left(u_{p}^{0}\right)+\sqrt{\varepsilon}\left\|v_{e}^{1}(\cdot)\right\|_{H^{3}} \leq C,
$$

thanks to bounds on the Euler flows, summarized in Section 2.3.1, Similarly, we have

$$
\begin{aligned}
\sup _{x} \int\left|y v_{s y}\right| d y & \leq C\left(v_{p}^{0}\right)+\varepsilon^{1 / 2} \sup _{x} \int\left|y v_{e z}^{1}(x, \sqrt{\varepsilon} y)\right| d y \\
& \leq C\left(v_{p}^{0}\right)+\varepsilon^{-1 / 2} \sup _{x} \int\left|z v_{e z}^{1}\right| d z \\
& \leq C\left(v_{p}^{0}\right)+C \varepsilon^{-1 / 2} L^{-1}\left(\iint\left|z v_{e z}^{1}\right| d x d z+\iint\left|z v_{e x z}^{1}\right| d x d z\right) \\
& \lesssim 1+C \varepsilon^{-1 / 2} L^{-1}\left\|\langle z\rangle^{n} v_{e}^{1}\right\|_{H^{2}}^{2},
\end{aligned}
$$

which is bounded by $C(L) \varepsilon^{-1 / 2}$, thanks to the estimates from Lemma 2.5. Also, we have

$$
\begin{aligned}
\sup _{x} \int y\left\{u_{s y}\right\}^{2} d y & \leq \sup _{x} \int y\left[\varepsilon\left|u_{e z}^{0}\right|^{2}+\left|u_{p y}^{0}\right|^{2}+\varepsilon^{2}\left|u_{e z}^{1}\right|^{2}\right] d y \\
& \leq C\left(u_{p}^{0}\right)+\sup _{x} \int\left[z\left|u_{e z}^{0}\right|^{2}+\varepsilon z\left|u_{e z}^{1}\right|^{2}\right] d z \\
& \leq C\left(u_{e}^{0}, u_{p}^{0}\right)+\varepsilon L^{-1}\left(\iint z\left|u_{e z}^{1}\right|^{2} d x d z+\iint z\left|u_{e x z}^{1}\right|^{2} d x d z\right) \\
& \lesssim 1+\varepsilon L^{-1}\left\|\langle z\rangle^{n} v_{e}^{1}\right\|_{H^{2}}^{2}
\end{aligned}
$$

which is again bounded by $C$, for $\varepsilon \ll L$. Putting this together into the above definition of $C\left(\varepsilon, L, u_{s}, v_{s}\right)$ and the fact that $\varepsilon \ll L$ yield the lemma at once.

\subsection{Positivity estimates}

In this section, we establish the following crucial positivity estimate:

Lemma 3.3. Let $[u, v]$ be the solution to the problem (3.1)-(3.3). Assume that $\varepsilon \ll L$. There holds

$$
\left\|\nabla_{\varepsilon} v\right\|_{L^{2}}^{2}+\varepsilon^{2} \int_{x=0} v_{x}^{2}+\varepsilon \int_{x=L} v_{y}^{2} \lesssim\left\|\nabla_{\varepsilon} u\right\|_{L^{2}}^{2}+L\left\|\nabla_{\varepsilon} v\right\|_{L^{2}}^{2}+\|f\|_{L^{2}}^{2}+\varepsilon\|g\|_{L^{2}}^{2} .
$$

Proof. We start from the identity: $\partial_{y}\left\{\frac{v}{u_{s}}\right\} \times(\underline{3.1})-\varepsilon \partial_{x}\left\{\frac{v}{u_{s}}\right\} \times(3.2)$. Formally, this is the vorticity 
equation multiplied by the test function $\frac{v}{u_{s}}$. This yields

$$
\begin{aligned}
& \iint \partial_{y}\left\{\frac{v}{u_{s}}\right\}\left\{u_{s} u_{x}+u_{s x} u+v_{s} u_{y}+v \partial_{y} u_{s}+p_{x}-\Delta_{\varepsilon} u\right\} \\
& -\iint \partial_{x}\left\{\frac{v}{u_{s}}\right\}\left\{\varepsilon\left[u_{s} v_{x}+u \partial_{x} v_{s}+v_{s} v_{y}+v \partial_{y} v_{s}\right]+p_{y}-\varepsilon \Delta_{\varepsilon} v\right\} \\
= & \iint \partial_{y}\left\{\frac{v}{u_{s}}\right\} f-\varepsilon \partial_{x}\left\{\frac{v}{u_{s}}\right\} g .
\end{aligned}
$$

Again, we use the inequality $|v| \leq \sqrt{y}\left\{\int_{0}^{y} v_{y}^{2}\right\}^{1 / 2}$, together with the estimates (3.6)-(3.8) on $\left[u_{s}, v_{s}\right]$ to estimate the right-hand side of (3.10). We have

$$
\begin{aligned}
& \iint\left\{\frac{v_{y}}{u_{s}}-\frac{v \partial_{y} u_{s}}{u_{s}^{2}}\right\} f-\varepsilon\left\{\frac{v_{x}}{u_{s}}-\frac{v \partial_{x} u_{s}}{u_{s}^{2}}\right\} g \\
\leq & \frac{\sup _{x}\left|\langle y\rangle^{1 / 2} \nabla_{\varepsilon} u_{s}\right|_{2}+\sup \left\|u_{s}\right\|_{\infty}}{\left\{\min u_{s}\right\}^{2}}\left\{\|f\|_{L^{2}}+\sqrt{\varepsilon}\|g\|_{L^{2}}\right\}\left\|\nabla_{\varepsilon} v\right\|_{L^{2}} \\
\lesssim & \left\{\|f\|_{L^{2}}+\sqrt{\varepsilon}\|g\|_{L^{2}}\right\}\left\|\nabla_{\varepsilon} v\right\|_{L^{2}},
\end{aligned}
$$

in which the Young inequality can be applied to absorb the $L^{2}$ norm of $\nabla_{\varepsilon} v$ to the left hand side of $(\underline{3.9})$.

Next, we treat each term on the left-hand side of (3.10). First, integrating by parts multiple times, we have

$$
\begin{aligned}
& \iint \partial_{y}\left\{\frac{v}{u_{s}}\right\}\left\{u_{s} u_{x}+v \partial_{y} u_{s}\right\}-\iint \partial_{x}\left\{\frac{v}{u_{s}}\right\} \varepsilon u_{s} v_{x} \\
= & \iint \partial_{y}\left\{\frac{v}{u_{s}}\right\}\left\{-u_{s} v_{y}+v \partial_{y} u_{s}\right\}-\iint\left\{\frac{v_{x}}{u_{s}}-\frac{\partial_{x} u_{s} v}{u_{s}^{2}}\right\} \varepsilon u_{s} v_{x} \\
= & \iint\left\{\frac{v_{y}}{u_{s}}-\frac{\partial_{y} u_{s} v}{u_{s}^{2}}\right\}\left\{-u_{s} v_{y}+v \partial_{y} u_{s}\right\}-\varepsilon \iint v_{x}^{2}+\iint \frac{\varepsilon \partial_{x} u_{s} v v_{x}}{u_{s}^{2}} \\
= & -\iint v_{y}^{2}+2 \iint v v_{y} \frac{\partial_{y} u_{s}}{u_{s}}-\iint \frac{\left\{\partial_{y} u_{s}\right\}^{2} v^{2}}{u_{s}^{2}}-\varepsilon \iint v_{x}^{2}+\iint \frac{\varepsilon \partial_{y} u_{s} v v_{x}}{u_{s}^{2}} \\
= & -\iint v_{y}^{2}-\iint \partial_{y}\left\{\frac{\partial_{y} u_{s}}{u_{s}}\right\} v^{2}-\iint v^{2} \frac{\left\{\partial_{y} u_{s}\right\}^{2}}{u_{s}^{2}}-\varepsilon \iint v_{x}^{2}+\iint \frac{\varepsilon \partial_{y} u_{s} v v_{x}}{u_{s}^{2}} \\
= & -\iint v_{y}^{2}-\iint \frac{\partial_{y y} u_{s}}{u_{s}} v^{2}-\varepsilon \iint v_{x}^{2}+\iint \frac{\varepsilon \partial_{y} u_{s} v v_{x}}{u_{s}^{2}} \\
= & -\iint u_{s}^{2}\left|\partial_{y}\left\{\frac{v}{u_{s}}\right\}\right|^{2}-\varepsilon \iint v_{x}^{2}+\iint \frac{\varepsilon \partial_{y} u_{s} v v_{x}}{u_{s}^{2}}
\end{aligned}
$$

in which the last equality is precisely due to the positivity estimate (1.16). From (1.17), we obtain a lower bound

$$
\iint \partial_{y}\left\{\frac{v}{u_{s}}\right\}\left\{u_{s} u_{x}+v \partial_{y} u_{s}\right\}-\iint \partial_{x}\left\{\frac{v}{u_{s}}\right\} \varepsilon u_{s} v_{x} \lesssim-\iint\left|\nabla_{\varepsilon} v\right|^{2}+\iint \frac{\varepsilon \partial_{y} u_{s} v v_{x}}{u_{s}^{2}},
$$

which crucially yields a bound on the $L^{2}$ norm of $\nabla_{\varepsilon} v$; or precisely, the $L^{2}$ norm of $\nabla_{\varepsilon} v$ appearing on the left-hand side of (3.9). 
Next, we treat the pressure term. Integrating by parts, with recalling that $p=2 \varepsilon u_{x}$ at $x=L$, we have

$$
\begin{aligned}
\iint \partial_{y}\left\{\frac{v}{u_{s}}\right\} p_{x}-\iint \partial_{x}\left\{\frac{v}{u_{s}}\right\} p_{y} & =\int_{x=L} \partial_{y}\left\{\frac{v}{u_{s}}\right\} p \\
& =2 \varepsilon \int_{x=L} \partial_{y}\left\{\frac{v}{u_{s}}\right\} u_{x}=-2 \varepsilon \int_{x=L} \partial_{y}\left\{\frac{v}{u_{s}}\right\} v_{y} \\
& =-2 \varepsilon \int_{x=L} \frac{v_{y}^{2}}{u_{s}}-2 \varepsilon \int_{x=L} \partial_{y}\left\{\frac{1}{u_{s}}\right\} v v_{y}
\end{aligned}
$$

in which we can estimate

$$
\begin{aligned}
\varepsilon \int_{x=L} \partial_{y}\left\{\frac{1}{u_{s}}\right\} v v_{y} & \leq \varepsilon \int_{x=L} \frac{\partial_{y} u_{s}}{u_{s}^{2}} v v_{y} \leq \varepsilon \sup \frac{\partial_{y} u_{s}}{u_{s}^{3 / 2}} \int_{x=L} v \frac{v_{y}}{\sqrt{u_{s}}} \\
& \leq\left\{C\left(u_{e y}^{0}, u_{p y}^{0}\right)+\varepsilon^{1 / 2}\left\|u_{e z}^{1}\right\|_{\infty}\right\} L \varepsilon\left\|v_{x}\right\|_{L^{2}}\left\{\int_{x=L} \frac{v_{y}^{2}}{u_{s}}\right\}^{1 / 2} .
\end{aligned}
$$

Here, thanks to the bounds on the Euler flows, summarized in Section 2.3.1, we in particular have $\varepsilon^{1 / 2}\left\|u_{e z}^{1}\right\|_{\infty} \leq C \varepsilon^{1 / 2}\left\|u_{e}^{1}\right\|_{H^{3}} \lesssim 1$. Together with the Young inequality, we thus obtain

$$
\iint\left[\partial_{y}\left\{\frac{v}{u_{s}}\right\} p_{x}-\partial_{x}\left\{\frac{v}{u_{s}}\right\} p_{y}\right] \leq-\frac{\varepsilon}{2} \int_{x=L} \frac{v_{y}^{2}}{u_{s}}+C\left(u_{s}, v_{s}\right) L^{2}\left\|\nabla_{\varepsilon} v\right\|_{L^{2}}^{2},
$$

in which we stress that the boundary term is favorable.

Next, we shall treat terms involving the Laplacian. Again, we recall from [13, 14, that the Stokes problem yields $[u, v] \in H^{3 / 2+}$ and $p \in H^{1 / 2+}$, and so their traces $[\nabla u, \nabla v] \in L^{2+}(\Gamma)$ and $p \in L^{2+}(\Gamma)$ on any smooth curve $\Gamma$. Moreover, $[u, v] \in H^{2}$ and $p \in H^{1}$ away from the four corners of $[0, L] \times[0, N]$. Hence, we can evaluate

$$
\begin{aligned}
I:= & \iint\left[-\partial_{y}\left\{\frac{v}{u_{s}}\right\} \Delta_{\varepsilon} u+\partial_{x}\left\{\frac{v}{u_{s}}\right\} \varepsilon \Delta_{\varepsilon} v\right] \\
= & \iint\left[-\partial_{y}\left\{\frac{v}{u_{s}}\right\}\left[u_{y y}+\varepsilon u_{x x}\right]+\partial_{x}\left\{\frac{v}{u_{s}}\right\} \varepsilon\left[v_{y y}+\varepsilon v_{x x}\right]\right] \\
= & \iint\left[-\left\{\frac{v_{y}}{u_{s}}-\frac{v \partial_{y} u_{s}}{u_{s}^{2}}\right\}\left[u_{y y}+\varepsilon u_{x x}\right]+\left\{\frac{v_{x}}{u_{s}}-\frac{v \partial_{x} u_{s}}{u_{s}^{2}}\right\} \varepsilon\left[v_{y y}+\varepsilon v_{x x}\right]\right] \\
= & \iint\left[\frac{u_{y y} u_{x}}{u_{s}}+\varepsilon \frac{u_{x x} u_{x}}{u_{s}}+\varepsilon \frac{v_{x} v_{y y}}{u_{s}}+\varepsilon^{2} \frac{v_{x} v_{x x}}{u_{s}}\right] \\
& +\iint\left[\left\{\frac{v \partial_{y} u_{s}}{u_{s}^{2}}\right\}\left[u_{y y}+\varepsilon u_{x x}\right]-\frac{v \partial_{x} u_{s}}{u_{s}^{2}}\left[\varepsilon v_{y y}+\varepsilon^{2} v_{x x}\right]\right] .
\end{aligned}
$$

Now taking integration by parts respectively in each integration above, with a special attention on 
the boundary contributions, we get

$$
\begin{aligned}
I= & -\frac{1}{2} \int_{x=L} \frac{u_{y}^{2}}{u_{s}}+\frac{1}{2} \iint \partial_{x}\left\{\frac{1}{u_{s}}\right\} u_{y}^{2}-\iint \partial_{y}\left\{\frac{1}{u_{s}}\right\} u_{y} u_{x} \\
& +\frac{\varepsilon}{2} \int_{x=L} \frac{u_{x}^{2}}{u_{s}}-\frac{\varepsilon}{2} \iint \partial_{x}\left\{\frac{1}{u_{s}}\right\} u_{x}^{2} \\
& -\frac{\varepsilon}{2} \int_{x=L} \frac{v_{y}^{2}}{u_{s}}+\frac{\varepsilon}{2} \iint \partial_{x}\left\{\frac{1}{u_{s}}\right\} v_{y}^{2}-\varepsilon \iint \partial_{y}\left\{\frac{1}{u_{s}}\right\} v_{y} v_{x} \\
& +\frac{\varepsilon^{2}}{2} \int_{x=L} \frac{v_{x}^{2}}{u_{s}}-\frac{\varepsilon^{2}}{2} \int_{x=0} \frac{v_{x}^{2}}{u_{s}}-\frac{\varepsilon^{2}}{2} \iint \partial_{x}\left\{\frac{1}{u_{s}}\right\} v_{x}^{2} \\
& -\iint \partial_{y}\left\{\frac{v \partial_{y} u_{s}}{u_{s}^{2}}\right\} u_{y}-\varepsilon \iint \partial_{x}\left\{\frac{v \partial_{y} u_{s}}{u_{s}^{2}}\right\} u_{x}+\varepsilon \int_{x=L}\left\{\frac{v \partial_{y} u_{s}}{u_{s}^{2}}\right\} u_{x} \\
& +\varepsilon \iint \partial_{y}\left\{\frac{v \partial_{x} u_{s}}{u_{s}^{2}}\right\} v_{y}+\varepsilon^{2} \iint \partial_{x}\left\{\frac{v \partial_{x} u_{s}}{u_{s}^{2}}\right\} v_{x}-\varepsilon^{2} \int_{x=L}\left\{\frac{v \partial_{y} u_{s}}{u_{s}^{2}}\right\} v_{x} .
\end{aligned}
$$

Let us first take care of boundary contributions. Notice that there is only one boundary term at $x=0$, which is a favorable term: $-\frac{\varepsilon^{2}}{2} \int_{x=0} \frac{v_{x}^{2}}{u_{s}}$. Now as for boundary terms at $x=L$, one can use the fact that $u_{y}+\varepsilon v_{x}=0$ and $u_{x}+v_{y}=0$ at $x=L$, and hence, the boundary contributions at $x=L$ can be simplified as

$$
\begin{aligned}
B^{L}:= & -\frac{1}{2} \int_{x=L} \frac{u_{y}^{2}}{u_{s}}+\frac{\varepsilon}{2} \int_{x=L} \frac{u_{x}^{2}}{u_{s}}-\frac{\varepsilon}{2} \int_{x=L} \frac{v_{y}^{2}}{u_{s}}+\frac{\varepsilon^{2}}{2} \int_{x=L} \frac{v_{x}^{2}}{u_{s}} \\
& +\varepsilon \int_{x=L}\left\{\frac{v \partial_{y} u_{s}}{u_{s}^{2}}\right\} u_{x}-\varepsilon^{2} \int_{x=L}\left\{\frac{v \partial_{y} u_{s}}{u_{s}^{2}}\right\} v_{x} \\
= & -\varepsilon \int_{x=L}\left\{\frac{v \partial_{y} u_{s}}{u_{s}^{2}}\right\} v_{y}-\varepsilon \int_{x=L}\left\{\frac{v \partial_{y} u_{s}}{u_{s}^{2}}\right\} u_{y} \\
= & -\varepsilon \int_{x=L}\left\{\frac{v \partial_{y} u_{s}}{u_{s}^{2}}\right\} v_{y}+\varepsilon \int_{x=L} \partial_{y}\left\{\frac{v \partial_{y} u_{s}}{u_{s}^{2}}\right\} u,
\end{aligned}
$$

which can be estimated by

$$
\begin{aligned}
B^{L} \leq & \varepsilon \sqrt{L} \sup _{x}\left|\frac{\partial_{y} u_{s}}{u_{s}}\right|\left\|v_{x}\right\|_{L^{2}} \sqrt{\int_{x=L} \frac{v_{y}^{2}}{u_{s}}}+\varepsilon \sqrt{L} \sup _{x}\left|\frac{\partial_{y} u_{s}}{u_{s}}\right|\left\|u_{x}\right\|_{L^{2}} \sqrt{\int_{x=L} \frac{v_{y}^{2}}{u_{s}}} \\
& +\varepsilon L\left\{\sup _{x} \sqrt{\int \frac{y\left|\partial_{y y} u_{s}\right|}{u_{s}^{2}} d y}+\sup _{x} \sqrt{\int \frac{y\left\{\partial_{y} u_{s}\right\}^{2}}{u_{s}^{3}}} d y\right\}\left\|u_{x}\right\|_{L^{2}} \sqrt{\int_{x=L} \frac{v_{y}^{2}}{u_{s}}} .
\end{aligned}
$$

We estimate norms on $u_{s}$ in the same lines as done in (3.6)-(3.8). Recall that $u_{s}=u_{e}^{0}+u_{p}^{0}+\sqrt{\varepsilon} u_{e}^{1}$ and $u_{s}$ is bounded below away from zero thanks to the assumption (1.14). Now, similarly as done 
for (3.8), we have

$$
\begin{aligned}
\sup _{x} \int y\left|u_{\text {syy }}\right| d y & \leq \sup _{x} \int y\left[\varepsilon\left|u_{e z z}^{0}\right|+\left|u_{\text {pyy }}^{0}\right|+\varepsilon^{3 / 2}\left|u_{e z z}^{1}\right|\right] d y \\
& \leq C\left(u_{p}^{0}\right)+\sup _{x} \int\left[z\left|u_{e z z}^{0}\right|+\varepsilon^{1 / 2} z\left|u_{e z z}^{1}\right|\right] d z \\
& \leq C\left(u_{e}^{0}, u_{p}^{0}\right)+\varepsilon^{1 / 2} L^{-1}\left(\iint z\left|u_{e z z}^{1}\right| d x d z+\iint z\left|u_{e x z z}^{1}\right| d x d z\right) \\
& \leq C\left(u_{e}^{0}, u_{p}^{0}\right)+\varepsilon^{1 / 2} L^{-1}\left\|\langle z\rangle^{n} v_{e}^{1}\right\|_{W^{3, q}} \\
& \lesssim 1+C(L) \varepsilon^{-1 / 2+1 / q},
\end{aligned}
$$

for any $q>1$. Here, Lemma 2.5 was used. Taking $q \rightarrow 1$ in the above estimates so that $-1 / 2+1 / q>$ 0 , the above is bounded uniformly by a constant $C\left(u_{s}, v_{s}\right)$, which is independent of small $\varepsilon, L$. The integral $\int y\left|u_{s y}\right|^{2} d y$ is already estimated in (3.8). Also, we get

$$
\left\|u_{s y}\right\|_{\infty} \leq \sqrt{\varepsilon}\left\|u_{e z}^{0}\right\|_{\infty}+\left\|u_{p y}^{0}\right\|_{\infty}+\varepsilon\left\|u_{e z}^{1}\right\|_{\infty} \leq C\left(u_{e}^{0}, u_{p}^{0}\right)+\varepsilon\left\|v_{e}^{1}(\cdot)\right\|_{H^{3}} \leq C .
$$

This together with the Young inequality yields

$$
B^{L} \leq \frac{\varepsilon}{4} \int_{x=L} \frac{v_{y}^{2}}{u_{s}}+C\left(u_{s}, v_{s}\right) L\left\|\nabla_{\varepsilon} v\right\|_{2}^{2},
$$

upon using the divergence-free condition $u_{x}=-v_{y}$. Here, $\nabla_{\varepsilon}=\left(\sqrt{\varepsilon} \partial_{x}, \partial_{y}\right)$. The boundary term in (3.16) can be absorbed into the good boundary term in (3.12).

We now combine the untreated terms on the left-hand side of (3.10), all the interior terms in (3.13), and the last term in (3.11), altogether. We shall use the standard embedding inequalities $\|u\|_{L^{2}} \leq L\left\|u_{x}\right\|_{L^{2}},|v| \leq \sqrt{y}\left\{\int_{0}^{y} v_{y}^{2}\right\}^{1 / 2}$ and $|u| \leq \sqrt{y}\left\{\int_{0}^{y} u_{y}^{2}\right\}^{1 / 2}$. Respectively, we have

$$
\begin{aligned}
R^{0}:= & \iint \frac{1}{2} \partial_{x}\left\{\frac{1}{u_{s}}\right\} u_{y}^{2}-\partial_{y}\left\{\frac{1}{u_{s}}\right\} u_{y} u_{x} \\
& \iint-\frac{\varepsilon}{2} \partial_{x}\left\{\frac{1}{u_{s}}\right\} u_{x}^{2}+\frac{\varepsilon}{2} \partial_{x}\left\{\frac{1}{u_{s}}\right\} v_{y}^{2}-\varepsilon \partial_{y}\left\{\frac{1}{u_{s}}\right\} v_{y} v_{x}-\frac{\varepsilon^{2}}{2} \partial_{x}\left\{\frac{1}{u_{s}}\right\} v_{x}^{2} \\
& -\iint \partial_{y}\left\{\frac{v \partial_{y} u_{s}}{u_{s}^{2}}\right\} u_{y}-\varepsilon \partial_{x}\left\{\frac{v \partial_{y} u_{s}}{u_{s}^{2}}\right\} u_{x}+\varepsilon \partial_{y}\left\{\frac{v \partial_{x} u_{s}}{u_{s}^{2}}\right\} v_{y}+\varepsilon^{2} \partial_{x}\left\{\frac{v \partial_{x} u_{s}}{u_{s}^{2}}\right\} v_{x} \\
& +\iint \partial_{y}\left\{\frac{v}{u_{s}}\right\}\left\{\partial_{x} u_{s} u+v_{s} u_{y}\right\}-\varepsilon \partial_{x}\left\{\frac{v}{u_{s}}\right\}\left\{u \partial_{x} v_{s}+v_{s} v_{y}+v \partial_{y} v_{s}\right\}+\frac{\varepsilon \partial_{y} u_{s} v v_{x}}{u_{s}^{2}} .
\end{aligned}
$$

Let us give estimates on each term on the right. We claim that

$$
R^{0} \lesssim\left\|\nabla_{\varepsilon} u\right\|_{L^{2}}^{2}+\left\|\nabla_{\varepsilon} u\right\|_{L^{2}}\left\|\nabla_{\varepsilon} v\right\|_{L^{2}}+C(L) \sqrt{\varepsilon}\left\|\nabla_{\varepsilon} v\right\|_{L^{2}}^{2}
$$

Proof of (3.17). First, we have

$$
\iint \frac{1}{2} \partial_{x}\left\{\frac{1}{u_{s}}\right\} u_{y}^{2}-\partial_{y}\left\{\frac{1}{u_{s}}\right\} u_{y} u_{x} \leq C\left\|\nabla u_{s}\right\|_{\infty}\left\|u_{y}\right\|_{L^{2}}\left(\left\|u_{y}\right\|_{L^{2}}+\left\|v_{y}\right\|_{L^{2}}\right)
$$


in which the bounds (3.5) and (3.15) gives $\left\|\nabla u_{s}\right\|_{\infty} \lesssim 1$. Next, upon recalling the definition $\nabla_{\varepsilon}=\left(\sqrt{\varepsilon} \partial_{x}, \partial_{y}\right)$, the Hölder inequality yields

$$
\begin{gathered}
\iint-\frac{\varepsilon}{2} \partial_{x}\left\{\frac{1}{u_{s}}\right\} u_{x}^{2}+\frac{\varepsilon}{2} \partial_{x}\left\{\frac{1}{u_{s}}\right\} v_{y}^{2}-\varepsilon \partial_{y}\left\{\frac{1}{u_{s}}\right\} v_{y} v_{x}-\frac{\varepsilon^{2}}{2} \partial_{x}\left\{\frac{1}{u_{s}}\right\} v_{x}^{2} \\
\leq C\left\|\nabla u_{s}\right\|_{\infty}\left(\left\|\nabla_{\varepsilon} u\right\|_{L^{2}}^{2}+\left\|\nabla_{\varepsilon} u\right\|_{L^{2}}\left\|\nabla_{\varepsilon} v\right\|_{L^{2}}+\sqrt{\varepsilon}\left\|\nabla_{\varepsilon} v\right\|_{L^{2}}^{2}\right)
\end{gathered}
$$

which again gives the bound as claimed, since $\left\|\nabla u_{s}\right\|_{\infty} \lesssim 1$. We now estimate the third line on the right of $R^{0}$. We first have

$$
\begin{aligned}
\iint \partial_{y}\left\{\frac{v \partial_{y} u_{s}}{u_{s}^{2}}\right\} u_{y} & =\iint\left\{\frac{\partial_{y} u_{s}}{u_{s}^{2}}\right\} u_{y} v_{y}+\iint \partial_{y}\left\{\frac{\partial_{y} u_{s}}{u_{s}^{2}}\right\} u_{y} v \\
& \leq C\left(\left\|u_{s y}\right\|_{\infty}+\sup _{x}\left(\int_{0}^{\infty} y\left(\left|u_{s y y}\right|^{2}+\left|u_{s y}\right|^{4}\right)\right)^{1 / 2}\right)\left\|u_{y}\right\|_{L^{2}}\left\|v_{y}\right\|_{L^{2}}
\end{aligned}
$$

and

$$
\begin{aligned}
\iint \varepsilon \partial_{x}\left\{\frac{v \partial_{y} u_{s}}{u_{s}^{2}}\right\} u_{x} & =-\varepsilon \iint\left\{\frac{\partial_{y} u_{s}}{u_{s}^{2}}\right\} v_{y} v_{x}-\varepsilon \iint \partial_{x}\left\{\frac{\partial_{y} u_{s}}{u_{s}^{2}}\right\} v_{y} v \\
& \leq C\left(\sqrt{\varepsilon}\left\|u_{s y}\right\|_{\infty}+\varepsilon \sup _{x}\left(\int_{0}^{\infty} y\left(\left|u_{s x y}\right|^{2}+\left|u_{s x} u_{s y}\right|^{2}\right)\right)^{1 / 2}\right)\left\|\nabla_{\varepsilon} v\right\|_{L^{2}}^{2} .
\end{aligned}
$$

The last two terms on the third line on the right of $R^{0}$ can be estimated very similarly. We give bounds on the norms of $\left[u_{s}, v_{s}\right]$. Similarly as done in (3.14), we get

$$
\begin{aligned}
\sup _{x} \int y\left|u_{s y y}\right|^{2} d y & \leq \sup _{x} \int y\left[\varepsilon^{2}\left|u_{e z z}^{0}\right|^{2}+\left|u_{p y y}^{0}\right|^{2}+\varepsilon^{3}\left|u_{e z z}^{1}\right|^{2}\right] d y \\
& \leq C\left(u_{p}^{0}\right)+\sup _{x} \int\left[\varepsilon z\left|u_{e z z}^{0}\right|^{2}+\varepsilon^{2} z\left|u_{e z z}^{1}\right|^{2}\right] d z \\
& \leq C\left(u_{e}^{0}, u_{p}^{0}\right)+\varepsilon^{2} L^{-1}\left(\iint z\left|u_{e z z}^{1}\right|^{2} d x d z+\iint z\left|u_{e x z z}^{1}\right|^{2} d x d z\right) \\
& \leq C\left(u_{e}^{0}, u_{p}^{0}\right)+\varepsilon^{2} L^{-1}\left\|\langle z\rangle^{n} v_{e}^{1}\right\|_{H^{3}}^{2} \\
& \lesssim 1+C(L) \varepsilon
\end{aligned}
$$

and

$$
\begin{aligned}
\sup _{x} \int y\left|u_{s y}\right|^{4} d y & \leq \sup _{x} \int y\left[\varepsilon^{2}\left|u_{e z}^{0}\right|^{4}+\left|u_{p y}^{0}\right|^{4}+\varepsilon^{4}\left|u_{e z}^{1}\right|^{4}\right] d y \\
& \leq C\left(u_{e}^{0}, u_{p}^{0}\right)+\varepsilon^{3} L^{-1}\left(\iint z\left|u_{e z}^{1}\right|^{4} d x d z+\iint z\left|u_{e x z}^{1}\right|^{4} d x d z\right) \\
& \leq C\left(u_{e}^{0}, u_{p}^{0}\right)+\varepsilon^{3} L^{-1}\left\|\langle z\rangle^{n} v_{e}^{1}\right\|_{W^{2,4}}^{2} \\
& \lesssim 1+C(L) \varepsilon^{3}
\end{aligned}
$$

both of which are thus bounded, thanks to the estimates from Lemma 2.5. Same bounds can be given for the weighted integrals of $\varepsilon\left|u_{s x y}\right|^{2}$ and $\varepsilon\left|u_{s x}\right|^{4}$, using the extra factor of $\varepsilon$ in these integrals. 
In addition, we have

$$
\begin{aligned}
\sup _{x} \int \varepsilon y\left|u_{s x x}\right| d y & \leq \sup _{x} \int \varepsilon y\left[\left|u_{p x x}^{0}\right|+\varepsilon^{1 / 2}\left|u_{e x x}^{1}\right|\right] d y \\
& \left.\leq C\left(u_{p}^{0}\right)+\varepsilon^{1 / 2} \sup _{x} \int z\left|v_{\text {exz }}^{1}\right|\right] d z \\
& \leq C\left(u_{p}^{0}\right)+\varepsilon^{1 / 2} L^{-1}\left(\iint z\left|v_{\text {exz }}^{1}\right| d x d z+\iint z\left|v_{\text {exxz }}^{1}\right| d x d z\right) \\
& \leq C\left(u_{e}^{0}, u_{p}^{0}\right)+\varepsilon^{1 / 2} L^{-1}\left\|\langle z\rangle^{n} v_{e}^{1}\right\|_{W^{3, q}}^{2} \\
& \lesssim 1+C(L) \varepsilon^{-3 / 2+2 / q},
\end{aligned}
$$

which are again bounded, thanks to the Euler bounds from Lemma 2.5, with $q$ being arbitrarily close to 1 .

We now give bounds on the last line on the right of $R^{0}$. We have

$$
\begin{aligned}
\iint \partial_{y} & \left\{\frac{v}{u_{s}}\right\}\left\{\partial_{x} u_{s} u+v_{s} u_{y}\right\} \\
= & \iint \partial_{y}\left\{\frac{1}{u_{s}}\right\}\left\{\partial_{x} u_{s} u+v_{s} u_{y}\right\} v+\iint\left\{\frac{1}{u_{s}}\right\}\left\{\partial_{x} u_{s} u+v_{s} u_{y}\right\} v_{y} \\
& \leq C\left(\sup _{x}\left(\int_{0}^{\infty} y\left(\left|u_{s y} u_{s x}\right|+\left|u_{s y} v_{s}\right|^{2}+\left|u_{s x}\right|^{2}\right)\right)^{1 / 2}+\left\|v_{s}\right\|_{\infty}\right)\left\|u_{y}\right\|_{L^{2}}\left\|v_{y}\right\|_{L^{2}}
\end{aligned}
$$

in which we note that $v_{s}$ is uniformly bounded. The estimate (3.8) gives the weighted bound on $u_{s y}$. Next, we estimate

$$
\begin{aligned}
\sup _{x} \int y\left\{u_{s x}\right\}^{2} d y & \leq \sup _{x} \int y\left[\left|u_{p x}^{0}\right|^{2}+\varepsilon\left|u_{e x}^{1}\right|^{2}\right] d y \\
& \leq C\left(u_{p}^{0}\right)+\sup _{x} \int z\left|v_{e z}^{1}\right|^{2} d z \\
& \leq C\left(u_{p}^{0}\right)+C\left(\int z\left|v_{e z}^{1}\right|_{\mid x=0}^{2} d z+\iint z\left|v_{e z}^{1}\right|^{2} d x d z+\iint z\left|v_{e x z}^{1}\right|^{2} d x d z\right), \\
& \lesssim 1 .
\end{aligned}
$$

This gives the desired bound on the first term on the last line in $R^{0}$. Next, we have

$$
\begin{aligned}
& \varepsilon \iint \partial_{x}\left\{\frac{v}{u_{s}}\right\}\left\{u \partial_{x} v_{s}+v_{s} v_{y}+v \partial_{y} v_{s}\right\} \\
& =\varepsilon \iint \partial_{x}\left\{\frac{1}{u_{s}}\right\}\left\{u \partial_{x} v_{s}+v_{s} v_{y}+v \partial_{y} v_{s}\right\} v+\varepsilon \iint\left\{\frac{1}{u_{s}}\right\}\left\{u \partial_{x} v_{s}+v_{s} v_{y}+v \partial_{y} v_{s}\right\} v_{x} \\
& \leq C \varepsilon \sup _{x}\left(\int_{0}^{\infty} y\left(\left|u_{s x} v_{s x}\right|+\left|u_{s x} v_{s}\right|^{2}+\left|u_{s x} v_{s y}\right|\right)\right)^{1 / 2}\left\|u_{y}\right\|_{L^{2}}\left\|v_{y}\right\|_{L^{2}} \\
& \quad+C(L) \varepsilon\left(\left\|v_{s x}\right\|_{\infty}+\left\|v_{s}\right\|_{\infty}+\sup _{x}\left(\int_{0}^{\infty} y\left|v_{s y}\right|^{2}\right)^{1 / 2}\right)\left\|u_{x}\right\|_{L^{2}}\left\|v_{x}\right\|_{L^{2}}
\end{aligned}
$$

in which the last estimate used the inequality: $\|u\|_{L^{2}} \leq L\left\|u_{x}\right\|_{L^{2}}$ and the divergence-free condition $v_{y}=-u_{x}$. As estimated above, it remains to give a uniform estimate on

$$
\left\|v_{s x}\right\|_{\infty} \leq\left\|v_{p x}^{0}\right\|_{\infty}+\left\|v_{e x}^{1}\right\|_{\infty} \lesssim 1+\left\|v_{e}^{1}\right\|_{W^{2, q}}^{2} \leq C(L)
$$


thanks to the estimates on $v_{e}^{1}$, with $q>2$. Finally, we estimate

$$
\varepsilon \iint \frac{\partial_{y} u_{s} v v_{x}}{u_{s}^{2}} \leq C \varepsilon \sup _{x}\left(\int_{0}^{\infty} y\left|u_{s y}\right|^{2}\right)^{1 / 2}\left\|v_{y}\right\|_{L^{2}}\left\|v_{x}\right\|_{L^{2}}
$$

in which the integral $\int y\left|u_{s y}\right|^{2} d y$ is already estimated in (3.8). Putting all above estimates together, we have completed the proof for the claim (3.17).

Finally, using the Young inequality and the smallness of $\varepsilon$, the $L^{2}$ norm of $\nabla_{\varepsilon} v$ can be absorbed into the left-hand side of (3.9).

This completes the proof of the positivity estimate and the lemma.

\subsection{Proof of Proposition 3.1}

The proof of Proposition 3.1 now follows straightforwardly from the energy estimate (Lemma 3.2) and the positivity estimate (Lemma 3.3), as a direct application of the Schaefer's fixed point theorem; see [4, Theorem 4, p. 504]. Indeed, first combining these estimates together and choosing $L$ sufficiently small, we get

$$
\left\|\nabla_{\varepsilon} u\right\|_{L^{2}}+\left\|\nabla_{\varepsilon} v\right\|_{L^{2}} \leq C\left(u_{s}, v_{s}\right)\left[\|f\|_{L^{2}}+\sqrt{\varepsilon}\|g\|_{L^{2}}\right]
$$

uniformly in $N$. Taking $N \rightarrow \infty$ yields the stability estimate (3.4).

To apply the fixed point theorem of Schaefer ([4, Theorem 4, p. 504]), we consider the following system

$$
\begin{aligned}
u+p_{x}-\Delta_{\varepsilon} u & =\lambda\left(f+u-\left[u_{s} u_{x}+u u_{s x}+v_{s} u_{y}+v u_{s y}\right]\right) \\
\frac{v}{\varepsilon}+\frac{p_{y}}{\varepsilon}-\Delta_{\varepsilon} v & =\lambda\left(g+\frac{v}{\varepsilon}-\left[u_{s} v_{x}+u v_{s x}+v_{s} v_{y}+v v_{s y}\right]\right) \\
u_{x}+v_{y} & =0
\end{aligned}
$$

or in the operator form $S[u, v]=\lambda T[u, v]$, for parameter $\lambda \in[0,1]$. The existence of a solution to (3.1)-(3.2) is equivalent to the existence of a fixed point of $S^{-1} T$. The compactness of the operator $S^{-1} T$ follows directly from that of the Stokes operator. To derive uniform bounds on the set of solutions $\left[u^{\lambda}, v^{\lambda}\right]$, we may rewrite the above system as

$$
\begin{aligned}
& (1-\lambda) u+\lambda\left[u_{s} u_{x}+u u_{s x}+v_{s} u_{y}+v u_{s y}\right]+p_{x}-\Delta_{\varepsilon} u=\lambda f \\
& (1-\lambda) \frac{v}{\varepsilon}+\lambda\left[u_{s} v_{x}+u v_{s x}+v_{s} v_{y}+v v_{s y}\right]+\frac{p_{y}}{\varepsilon}-\Delta_{\varepsilon} v=\lambda g
\end{aligned}
$$

together with the divergence-free condition and the same boundary conditions (3.3). The uniform estimates now follow almost identically from the above energy estimates and positivity estimates. We omit to repeat the details. This completes the proof of Proposition 3.1.

\section{$4 \quad L^{\infty}$ estimates}

In order to perform nonlinear iteration, we shall need to derive bounds in $L^{\infty}$ for the solution. We prove the following: 
Lemma 4.1. Consider the scaled Stokes system

$$
p_{x}-\Delta_{\varepsilon} u=f, \quad \frac{p_{y}}{\varepsilon}-\Delta_{\varepsilon} v=g
$$

together with the divergence-free condition $u_{x}+v_{y}=0$ and the (same) boundary conditions

$$
\begin{array}{rlrl}
{[u, v]_{y=0}=0} & \text { (no-slip), } & {[u, v]_{x=0}=0 \quad \text { (Dirichlet) },} \\
p-2 \varepsilon u_{x}=0, \quad u_{y}+\varepsilon v_{x}=0 & \text { at } x=L \text { (Neumann or stress-free). }
\end{array}
$$

Then, there holds

$$
\varepsilon^{\frac{\gamma}{4}}\|u\|_{\infty}+\varepsilon^{\frac{\gamma}{4}+\frac{1}{2}}\|v\|_{\infty} \lesssim C_{\gamma, L}\left\{\|u\|_{H^{1}}+\sqrt{\varepsilon}\|v\|_{H^{1}}+\|f\|_{L^{2}}+\sqrt{\varepsilon}\|g\|_{L^{2}}\right\},
$$

for some constant $C_{\gamma, L}$.

Proof. Since our rectangle domain can be covered by two $C^{0,1}$ charts, we may apply the standard extension theorem (see, for instance, [3, Theorem 5.4]) such that there exists $\bar{u} \in H^{1+\beta}\left(\mathbb{R}^{2}\right)$ and $\bar{v} \in H^{1+\beta}\left(\mathbb{R}^{2}\right)$, for $\beta \in(0,1)$, such that $[\bar{u}, \bar{v}]=[u, v]$ in $[0, L] \times \mathbb{R}_{+}$, and

$$
\|\bar{u}\|_{H^{1+\beta}} \leq C_{\beta, L}\|u\|_{H^{1+\beta}}, \quad\|\bar{v}\|_{H^{1+\beta}} \leq C_{\beta, L}\|v\|_{H^{1+\beta}},
$$

for some constant $C_{\beta, L}$ that depends only on $\beta$ and $L$. By the Sobolev's imbedding in $\mathbb{R}^{2}$ and an interpolation inequality for $\bar{u}$ and $\bar{v}$, we have for any $0<\tau<\alpha$,

$$
\begin{aligned}
\|u\|_{\infty} & \leq\|\bar{u}\|_{\infty} \leq\|\bar{u}\|_{H^{1+\tau}} \\
& \leq C_{\tau, L}\left[\|\bar{u}\|_{H^{1}}\right]^{\frac{\alpha-\tau}{\alpha}}\left[\|\bar{u}\|_{H^{1+\alpha}}\right]^{\frac{\tau}{\alpha}} \\
& \leq C_{\tau, \alpha, L}\left[\|u\|_{H^{1}}\right]^{\frac{\alpha-\tau}{\alpha}}\left[\|u\|_{H^{1+\alpha}}\right]^{\frac{\tau}{\alpha}}
\end{aligned}
$$

and similarly,

$$
\varepsilon^{1 / 2}\|v\|_{\infty} \leq C_{\tau, \alpha, L}\left[\varepsilon^{1 / 2}\|v\|_{H^{1}}\right]^{\frac{\alpha-\tau}{\alpha}}\left[\varepsilon^{1 / 2}\|v\|_{H^{1+\alpha}}\right]^{\frac{\tau}{\alpha}}
$$

for some constant $C_{\tau, \alpha, L}$. Here, we have used the standard interpolation between Sobolev spaces $H^{1}, H^{1+\tau}$, and $H^{1+\alpha}$, with $0<\tau<\alpha$. We note that thanks to our uniform estimates for $\|u\|_{H^{1}}$ and $\varepsilon^{1 / 2}\|v\|_{H^{1}}$, it suffices to give estimates on the $H^{1+\alpha}$ norm of $[u, \sqrt{\varepsilon} v]$.

In what follows, we fix $\alpha \sim 1 / 2$ and take $\tau$ so that $\tau<<\alpha$. We claim that there exists a possibly large number $m_{\alpha}>0$ such that there holds

$$
\|u\|_{H^{1+\alpha}}+\sqrt{\varepsilon}\|v\|_{H^{1+\alpha}} \lesssim \varepsilon^{-m_{\alpha}}\left\{\|f\|_{L^{2}}+\sqrt{\varepsilon}\|g\|_{L^{2}}+\|u\|_{H^{1}}+\sqrt{\varepsilon}\|v\|_{H^{1}}\right\} .
$$

Given the claim, we then have

$$
\|u\|_{\infty}+\sqrt{\varepsilon}\|v\|_{\infty} \leq C_{\tau, \alpha, L} \varepsilon^{-\frac{m_{\alpha} \tau}{\alpha}}\left\{\|f\|_{L^{2}}+\sqrt{\varepsilon}\|g\|_{L^{2}}+\|u\|_{H^{1}}+\sqrt{\varepsilon}\|v\|_{H^{1}}\right\} .
$$

The lemma would then be proved at once by choosing $\tau \ll \alpha$ so that $\frac{m_{\alpha} \tau}{\alpha} \leq \frac{\gamma}{4}$.

We shall now prove the claim for $\alpha=1 / 2$ and $m_{\alpha}=7 / 4$. To do so, let us introduce the (original) scaling:

$$
u_{\varepsilon}(x, y) \equiv u\left(L x, L \frac{y}{\sqrt{\varepsilon}}\right), \quad v_{\varepsilon}(x, y) \equiv \sqrt{\varepsilon} v\left(L x, L \frac{y}{\sqrt{\varepsilon}}\right), \quad p_{\varepsilon}(x, y) \equiv \frac{L}{\varepsilon} p\left(L x, L \frac{y}{\sqrt{\varepsilon}}\right) .
$$


Clearly, direct calculations yield $u_{\varepsilon x}+v_{\varepsilon y}=0$ and

$$
\begin{array}{rlrl}
\partial_{x x} u_{\varepsilon}(x, y) & =L^{2} u_{x x}\left(L x, L \frac{y}{\sqrt{\varepsilon}}\right), & \partial_{y y} u_{\varepsilon}(x, y)=\frac{L^{2}}{\varepsilon} u_{y y}\left(L x, L \frac{y}{\sqrt{\varepsilon}}\right) \\
\partial_{x x} v_{\varepsilon}(x, y)=L^{2} \sqrt{\varepsilon} v_{x x}\left(L x, L \frac{y}{\sqrt{\varepsilon}}\right), & \partial_{y y} v_{\varepsilon}(x, y)=\frac{L^{2}}{\varepsilon^{1 / 2}} v_{y y}\left(L x, L \frac{y}{\sqrt{\varepsilon}}\right) \\
\partial_{x} p_{\varepsilon}(x, y)=\frac{L^{2}}{\varepsilon} p_{x}\left(L x, L \frac{y}{\sqrt{\varepsilon}}\right), & \partial_{y} p_{\varepsilon}(x, y)=\frac{L^{2}}{\varepsilon^{3 / 2}} p_{y}\left(L x, L \frac{y}{\sqrt{\varepsilon}}\right) .
\end{array}
$$

Plugging these in the Stokes problem, we yield a normalized Stokes system:

$$
p_{\varepsilon x}-\Delta u_{\varepsilon}=\varepsilon^{-1} L^{2} f\left(L x, L \frac{y}{\sqrt{\varepsilon}}\right), \quad p_{\varepsilon y}-\Delta v_{\varepsilon}=\varepsilon^{-\frac{1}{2}} L^{2} g\left(L x, L \frac{y}{\sqrt{\varepsilon}}\right)
$$

in a fixed domain $0 \leq x \leq 1$ and $0 \leq y \leq \infty$ with boundary conditions

$$
\begin{aligned}
{\left[u_{\varepsilon}, v_{\varepsilon}\right] } & =0 \text { on both boundaries: }\{y=0\} \text { and }\{x=0\} \\
p_{\varepsilon}+2 u_{\varepsilon x} & =0, \quad v_{\varepsilon x}+u_{\varepsilon y}=0 \text { at } x=1 .
\end{aligned}
$$

We now invoke the standard elliptic estimate for the Stoke problem in such a fixed domain. Recall that there holds the Poincare's inequality: $\left\|u_{\varepsilon}\right\|_{L^{2}} \leq\left\|u_{\varepsilon x}\right\|_{L^{2}},\left\|v_{\varepsilon}\right\|_{L^{2}} \leq\left\|v_{\varepsilon x}\right\|_{L^{2}}$. Next, the standard energy estimates yield

$$
\begin{aligned}
\left\|\nabla u_{\varepsilon}\right\|_{L^{2}}+\left\|\nabla v_{\varepsilon}\right\|_{L^{2}} & \leq C\left\{\varepsilon^{-1} L^{2}\left\|f\left(L x, \frac{L y}{\sqrt{\varepsilon}}\right)\right\|_{L^{2}}+\varepsilon^{-\frac{1}{2}} L^{2}\left\|g\left(L x, \frac{L y}{\sqrt{\varepsilon}}\right)\right\|_{L^{2}}\right\} \\
& \leq C \varepsilon^{-3 / 4} L\left[\|f\|_{L^{2}}+\sqrt{\varepsilon}\|g\|_{L^{2}}\right] .
\end{aligned}
$$

Next, let us give an $L^{2}$ estimate on the pressure $p_{\varepsilon}$. First, for $h \in L^{2}$, we show that there is a vector-valued function $\phi \in H^{1}$ such that $\phi(0, y) \equiv 0, \phi(x, 0) \equiv 0, \nabla \cdot \phi=h$ and $\|\phi\|_{H^{1}} \lesssim\|h\|_{L^{2}}$. Indeed, we can decompose $h=\sum_{n=0}^{\infty} \mathbf{1}_{n \leq y<n+1}(y) h$, for characteristic functions $\mathbf{1}_{n \leq y<n+1}(y)$. Hence, for each $n$, the function $q_{n}=\mathbf{1}_{n \leq y<n+1}(y) h$ is supported in a unit square $[0,1] \times[n, n+1]$. By [13, page 27], we can then find $\phi_{n} \in H^{1}$, such that $\nabla \cdot \phi_{n}=h_{n}$ on the unit square, with $\phi_{n}(0, y) \equiv \phi_{n}(x, n) \equiv \phi_{n}(x, n+1) \equiv 0$. Furthermore, we have $\left\|\phi_{n}\right\|_{H^{1}} \leq C\left\|h_{n}\right\|_{L^{2}}$, uniformly in $n$. If we now define $\phi \equiv \sum_{n=1}^{\infty} \phi_{n}$, it then follows that $\|\phi\|_{H^{1}} \leq C\|h\|_{L^{2}}, \nabla \cdot \phi=h$ and $\phi(0, y) \equiv 0$, $\phi(x, 0) \equiv 0$.

The pressure estimate now follows directly from the existence of the vector field $\phi$. Indeed, we approximate $p_{\varepsilon}$ by smooth functions of the form $q=\nabla \cdot \phi$ so that $\left\|p_{\varepsilon}\right\|_{L^{2}} \lesssim\|q\|_{L^{2}}=\|\nabla \phi\|_{L^{2}}$. Then, we can use the vector field $\phi$ as a test function to the Stokes problem. This, together with the Young inequality, immediately yields

$$
\begin{aligned}
\left\|p_{\varepsilon}\right\|_{L^{2}} & \leq C\left\{\left\|\nabla u_{\varepsilon}\right\|_{L^{2}}+\left\|\nabla v_{\varepsilon}\right\|_{L^{2}}+\varepsilon^{-1} L^{2}\left\|f\left(L x, \frac{L y}{\sqrt{\varepsilon}}\right)\right\|_{L^{2}}+\varepsilon^{-\frac{1}{2}} L^{2}\left\|g\left(L x, \frac{L y}{\sqrt{\varepsilon}}\right)\right\|_{L^{2}}\right\} \\
& \leq C \varepsilon^{-3 / 4} L\left[\|f\|_{L^{2}}+\sqrt{\varepsilon}\|g\|_{L^{2}}\right]
\end{aligned}
$$

thanks to the estimate (4.5). 
Now, it remains to derive estimates in the higher regularity norms. We multiply the Stokes system by an arbitrary cut-off function $\chi\left(x, \frac{y}{\sqrt{\varepsilon}}\right)$ to obtain:

$$
\begin{aligned}
& \left\{\chi p_{\varepsilon}\right\}_{x}-\Delta\left\{\chi u_{\varepsilon}\right\}=\chi_{x} p_{\varepsilon}+u_{\varepsilon} \Delta \chi+2 \nabla \chi \cdot \nabla u_{\varepsilon}+\chi \varepsilon^{-1} L^{2} f \\
& \left\{\chi p_{\varepsilon}\right\}_{y}-\Delta\left\{\chi v_{\varepsilon}\right\}=\chi_{y} p_{\varepsilon}+v_{\varepsilon} \Delta \chi+2 \nabla \chi \cdot \nabla v_{\varepsilon}+\chi \varepsilon^{-\frac{1}{2}} L^{2} g
\end{aligned}
$$

If we choose $\chi=\chi_{1}\left(x, \frac{y}{\sqrt{\varepsilon}}\right)$ which has a compact support away from the corners, then the Stokes problem has an $H^{2}$ estimate so that

$$
\begin{aligned}
& \left\|\chi_{1} p_{\varepsilon}\right\|_{H^{1}}+\left\|\chi_{1} u_{\varepsilon}\right\|_{H^{2}}+\left\|\chi_{1} v_{\varepsilon}\right\|_{H^{2}} \\
\leq & \left\|\chi_{x} p_{\varepsilon}+u_{\varepsilon} \Delta \chi+2 \nabla \chi \cdot \nabla u_{\varepsilon}+\chi \varepsilon^{-1} L^{2} f\right\|_{L^{2}}+\left\|\chi_{y} p_{\varepsilon}+v_{\varepsilon} \Delta \chi+2 \nabla \chi \cdot \nabla v_{\varepsilon}+\chi \varepsilon^{-\frac{1}{2}} L^{2} g\right\|_{L^{2}} \\
\leq & \varepsilon^{-1}\left\{\left\|u_{\varepsilon}\right\|_{L^{2}}+\left\|v_{\varepsilon}\right\|_{L^{2}}\right\}+\varepsilon^{-\frac{1}{2}}\left\{\left\|\nabla u_{\varepsilon}\right\|_{L^{2}}+\left\|\nabla v_{\varepsilon}\right\|_{L^{2}}+\left\|p_{\varepsilon}\right\|_{L^{2}}\right\}+\varepsilon^{-3 / 4} L\|f\|_{L^{2}}+\varepsilon^{-1 / 4} L\|g\|_{L^{2}} \\
\leq & \varepsilon^{-5 / 4} L\left\{\|f\|_{L^{2}}+\sqrt{\varepsilon}\|g\|_{L^{2}}\right\},
\end{aligned}
$$

upon using the estimate (4.5). This implies the following uniform estimate for the unscaled solution $[u, v, p]$ via the change of variables (4.3):

$$
\begin{aligned}
\left\|\chi_{1} u_{\varepsilon}\right\|_{H^{2}} & \approx \varepsilon^{-1+\frac{1}{4}}\left\|\nabla^{2}\left(\chi_{1} u\right)\right\|_{L^{2}}+\varepsilon^{-\frac{1}{2}+\frac{1}{4}}\left\|\nabla\left(\chi_{1} u\right)\right\|_{L^{2}}+\varepsilon^{1 / 4}\left\|\chi_{1} u\right\|_{L^{2}}, \\
\left\|\chi_{1} v_{\varepsilon}\right\|_{H^{2}} & \approx \varepsilon^{1 / 2} \varepsilon^{-1+\frac{1}{4}}\left\|\nabla^{2}\left(\chi_{1} v\right)\right\|_{L^{2}}+\varepsilon^{\frac{1}{4}}\left\|\nabla\left(\chi_{1} v\right)\right\|_{L^{2}}+\varepsilon^{1 / 2+1 / 4}\left\|\chi_{1} v\right\|_{L^{2}}
\end{aligned}
$$

which immediately yield

$$
\begin{aligned}
& \varepsilon^{-1+\frac{1}{4}}\left\|\nabla^{2}\left(\chi_{1} u\right)\right\|_{L^{2}}+\varepsilon^{\frac{1}{2}} \varepsilon^{-1+\frac{1}{4}}\left\|\nabla^{2}\left(\chi_{1} v\right)\right\|_{L^{2}} \\
& \quad \leq \varepsilon^{-5 / 4} L\left\{\|f\|_{L^{2}}+\sqrt{\varepsilon}\|g\|_{L^{2}}\right\}+\varepsilon^{-1 / 4}\left\{\|u\|_{H^{1}}+\sqrt{\varepsilon}\|v\|_{H^{1}}\right\} .
\end{aligned}
$$

Next, we choose the cut-off function $\chi=\chi_{2}\left(x, \frac{y}{\sqrt{\varepsilon}}\right)$ with support near the corners, around which we do not have any $H^{2}$ estimate of the solution. However, thanks to [14], we do have a weaker estimate: precisely,

$$
\begin{aligned}
\left\|\chi_{2} p_{\varepsilon}\right\|_{H^{3 / 2}}+\left\|\chi_{2} u_{\varepsilon}\right\|_{H^{1+3 / 2}}+\left\|\chi_{2} v_{\varepsilon}\right\|_{H^{1+3 / 2}} \leq & \left\|\chi_{2 x} p_{\varepsilon}+\Delta \chi_{2} u_{\varepsilon}+2 \nabla \chi_{2} \cdot \nabla u_{\varepsilon}+\chi_{2} f\right\|_{L^{2}} \\
& +\left\|\chi_{2 y} p_{\varepsilon}+\Delta \chi_{2} v_{\varepsilon}+2 \nabla \chi_{2} \cdot \nabla v^{\varepsilon}+\chi_{2} g\right\|_{L^{2}} \\
\leq & \varepsilon^{-1}\left\{\varepsilon^{-3 / 4} L\|f\|_{L^{2}}+\varepsilon^{-1 / 4} L\|g\|_{L^{2}}\right\} .
\end{aligned}
$$

Noting that by scaling via (4.3) there hold

$$
\left\|\nabla\left\{\chi_{2} u_{\varepsilon}\right\}\right\|_{H^{1 / 2}}=\varepsilon^{-\frac{3}{4}+\frac{1}{4}}\left\|\nabla\left\{\chi_{2} u\right\}\right\|_{H^{1 / 2}}, \quad\left\|\nabla\left\{\chi_{2} v_{\varepsilon}\right\}\right\|_{H^{1 / 2}}=\varepsilon^{\frac{1}{2}} \varepsilon^{-\frac{3}{4}+\frac{1}{4}}\left\|\nabla\left\{\chi_{2} v\right\}\right\|_{H^{1 / 2}}
$$

we thus obtain

$$
\varepsilon^{-\frac{3}{2}+\frac{1}{4}}\left\|\nabla\left\{\chi_{2} u\right\}\right\|_{H^{1 / 2}}+\varepsilon^{\frac{1}{2}} \varepsilon^{-\frac{3}{4}+\frac{1}{4}}\left\|\nabla\left\{\chi_{2} v\right\}\right\|_{H^{1 / 2}} \leq \varepsilon^{-1}\left\{\varepsilon^{-3 / 4} L\|f\|_{L^{2}}+\varepsilon^{-1 / 4} L\|g\|_{L^{2}}\right\} .
$$

Finally, combining the estimates on $\chi_{1}[u, v]$ and $\chi_{2}[u, v]$ yields at once the claimed bound (4.2) for $\alpha=1 / 2$ and $m_{\alpha}=7 / 4$. The lemma is thus proved. 


\section{Proof of the main theorem}

We are now ready to give the proof of our main theorem. Consider the nonlinear scaled NavierStokes equations (1.4) and write the solutions $\left[U^{\varepsilon}, V^{\varepsilon}, P^{\varepsilon}\right]$ in the asymptotic expansion (1.9):

$$
\left[U^{\varepsilon}, V^{\varepsilon}, P^{\varepsilon}\right](x, y)=\left[u_{\text {app }}, v_{\text {app }}, p_{\text {app }}\right](x, y)+\varepsilon^{\gamma+\frac{1}{2}}\left[u^{\varepsilon}, v^{\varepsilon}, p^{\varepsilon}\right](x, y)
$$

with the approximate solutions constructed as in Proposition 2.1. We shall now study the equations for the remainder solutions $\left[u^{\varepsilon}, v^{\varepsilon}, p^{\varepsilon}\right]$. Let us recall the leading approximate solutions (without having the Prandtl layers $\left.\left[u_{p}^{1}, v_{p}^{1}\right]\right)$ :

$$
u_{s}(x, y)=u_{e}^{0}+u_{p}^{0}+\sqrt{\varepsilon} u_{e}^{1}, \quad v_{s}(x, y)=v_{p}^{0}+v_{e}^{1} .
$$

Then, the remainder solutions $\left[u^{\varepsilon}, v^{\varepsilon}, p^{\varepsilon}\right]$ solve

$$
\begin{aligned}
u_{s} u_{x}^{\varepsilon}+u^{\varepsilon} u_{s x}+v_{s} u_{y}^{\varepsilon}+v^{\varepsilon} u_{s y}+p_{x}^{\varepsilon}-\Delta_{\varepsilon} u^{\varepsilon} & =R_{1}\left(u^{\varepsilon}, v^{\varepsilon}\right) \\
u_{s} v_{x}^{\varepsilon}+u^{\varepsilon} v_{s x}+v_{s} v_{y}^{\varepsilon}+v^{\varepsilon} v_{s y}+\frac{p_{y}^{\varepsilon}}{\varepsilon}-\Delta_{\varepsilon} v^{\varepsilon} & =R_{2}\left(u^{\varepsilon}, v^{\varepsilon}\right) \\
u_{x}^{\varepsilon}+v_{y}^{\varepsilon} & =0
\end{aligned}
$$

in which $\Delta_{\varepsilon}=\partial_{y}^{2}+\varepsilon \partial_{x}^{2}$ and the remainders $R_{1}\left(u^{\varepsilon}, v^{\varepsilon}\right), R_{2}\left(u^{\varepsilon}, v^{\varepsilon}\right)$ are defined by

$$
\begin{aligned}
& R_{1}\left(u^{\varepsilon}, v^{\varepsilon}\right):=\varepsilon^{-\gamma-\frac{1}{2}} R_{\mathrm{app}}^{u}-\sqrt{\varepsilon}\left[\left(u_{p}^{1}+\varepsilon^{\gamma} u^{\varepsilon}\right) u_{x}^{\varepsilon}+u^{\varepsilon} u_{p x}^{1}+\left(v_{p}^{1}+\varepsilon^{\gamma} v^{\varepsilon}\right) u_{y}^{\varepsilon}+v^{\varepsilon} u_{p y}^{1}\right] \\
& R_{2}\left(u^{\varepsilon}, v^{\varepsilon}\right):=\varepsilon^{-\gamma-\frac{1}{2}} R_{\mathrm{app}}^{v}-\sqrt{\varepsilon}\left[\left(u_{p}^{1}+\varepsilon^{\gamma} u^{\varepsilon}\right) v_{x}^{\varepsilon}+u^{\varepsilon} v_{p x}^{1}+\left(v_{p}^{1}+\varepsilon^{\gamma} v^{\varepsilon}\right) v_{y}^{\varepsilon}+v^{\varepsilon} v_{p y}^{1}\right] .
\end{aligned}
$$

Here, the errors $R_{\mathrm{app}}^{u}, R_{\mathrm{app}}^{v}$ from the approximation are estimated in Proposition 2.1 ,

We shall apply the standard contraction mapping theorem for the existence of the solutions to the nonlinear problem. Indeed, we introduce the function space $X$, endowed with the norm:

$$
\left\|\left[u^{\varepsilon}, v^{\varepsilon}\right]\right\|_{X} \equiv\left\|\nabla_{\varepsilon} u^{\varepsilon}\right\|_{L^{2}}+\left\|\nabla_{\varepsilon} v^{\varepsilon}\right\|_{L^{2}}+\varepsilon^{\frac{\gamma}{2}}\left\|u^{\varepsilon}\right\|_{\infty}+\varepsilon^{\frac{1}{2}+\frac{\gamma}{2}}\left\|v^{\varepsilon}\right\|_{\infty} .
$$

Now, for each $\left[u^{\varepsilon}, v^{\varepsilon}\right] \in X$, we solve the following linear problem for $\left[\bar{u}^{\varepsilon}, \bar{v}^{\varepsilon}\right]$ :

$$
\begin{aligned}
& u_{s} \bar{u}_{x}^{\varepsilon}+\bar{u}^{\varepsilon} u_{s x}+v_{s} \bar{u}_{y}^{\varepsilon}+\bar{v}^{\varepsilon} u_{s y}+\bar{p}_{x}^{\varepsilon}-\Delta_{\varepsilon} \bar{u}^{\varepsilon}=R_{1}\left(u^{\varepsilon}, v^{\varepsilon}\right), \\
& u_{s} \bar{v}_{x}^{\varepsilon}+\bar{u}^{\varepsilon} v_{s x}+v_{s} \bar{v}_{y}^{\varepsilon}+\bar{v}^{\varepsilon} v_{s y}+\frac{\bar{p}_{y}^{\varepsilon}}{\varepsilon}-\Delta_{\varepsilon} \bar{v}^{\varepsilon}=R_{2}\left(u^{\varepsilon}, v^{\varepsilon}\right) .
\end{aligned}
$$

We are in the position to use the linear stability estimates obtained in Proposition 3.1, yielding

$$
\left\|\nabla_{\varepsilon} \bar{u}^{\varepsilon}\right\|_{L^{2}}+\left\|\nabla_{\varepsilon} \bar{v}^{\varepsilon}\right\|_{L^{2}} \leq\left\|R_{1}\left(u^{\varepsilon}, v^{\varepsilon}\right)\right\|_{L^{2}}+\sqrt{\varepsilon}\left\|R_{2}\left(u^{\varepsilon}, v^{\varepsilon}\right)\right\|_{L^{2}} .
$$

We give estimates on the remainders $R_{1}, R_{2}$, defined as in (5.4). Proposition 2.1 yields

$$
\varepsilon^{-\gamma-\frac{1}{2}}\left[\left\|R_{\mathrm{app}}^{u}\right\|_{L^{2}}+\sqrt{\varepsilon}\left\|R_{\mathrm{app}}^{v}\right\|_{L^{2}}\right] \leq C\left(L, u_{s}, v_{s}\right) \varepsilon^{-\gamma-\kappa+\frac{1}{4}}
$$


for arbitrary $\kappa>0$. In what follows, we take any $\gamma<1$ and take $\kappa$ so that $\gamma+\kappa<1 / 4$. In addition, by a view of the definition of $\|\cdot\|_{X}$, the divergence-free condition $u_{x}=-v_{y}$, and the fact that $\left[u_{p}^{1}, v_{p}^{1}\right]$ are nearly bounded, we estimate

$$
\begin{aligned}
\sqrt{\varepsilon}\left\|\left(u_{p}^{1}+\varepsilon^{\gamma} u^{\varepsilon}\right) u_{x}^{\varepsilon}\right\|_{L^{2}} & \leq \sqrt{\varepsilon}\left(\left\|u_{p}^{1}\right\|_{\infty}+\varepsilon^{\gamma}\left\|u^{\varepsilon}\right\|_{\infty}\right)\left\|u_{x}^{\varepsilon}\right\|_{L^{2}} \\
& \leq \varepsilon^{1 / 2-\kappa}\left(C\left(u_{p}^{1}\right)+\varepsilon^{\frac{\gamma}{2}}\left\|\left[u^{\varepsilon}, v^{\varepsilon}\right]\right\|_{X}\right)\left\|\left[u^{\varepsilon}, v^{\varepsilon}\right]\right\|_{X} \\
\sqrt{\varepsilon}\left\|\left(v_{p}^{1}+\varepsilon^{\gamma} v^{\varepsilon}\right) u_{y}^{\varepsilon}\right\|_{L^{2}} & \leq \sqrt{\varepsilon}\left(\left\|v_{p}^{1}\right\|_{\infty}+\varepsilon^{\gamma}\left\|v^{\varepsilon}\right\|_{\infty}\right)\left\|u_{y}^{\varepsilon}\right\|_{L^{2}} \\
& \leq\left(C\left(v_{p}^{1}\right) \varepsilon^{1 / 2-\kappa}+\varepsilon^{\frac{\gamma}{2}}\left\|\left[u^{\varepsilon}, v^{\varepsilon}\right]\right\|_{X}\right)\left\|\left[u^{\varepsilon}, v^{\varepsilon}\right]\right\|_{X} .
\end{aligned}
$$

Also, using the inequality $\left|\left[u^{\varepsilon}, v^{\varepsilon}\right]\right| \leq \sqrt{y}\left\|\left[u_{y}^{\varepsilon}, v_{y}^{\varepsilon}\right]\right\|_{L^{2}\left(\mathbb{R}_{+}\right)}$and the uniform $H^{1}$ bounds obtained in (2.56) on $u_{p}^{1}$, we get

$$
\begin{aligned}
\sqrt{\varepsilon}\left\|u^{\varepsilon} u_{p x}^{1}+v^{\varepsilon} u_{p y}^{1}\right\|_{L^{2}} & \leq \sqrt{\varepsilon} \sup _{x}\left(\left\|\langle y\rangle^{n} u_{p x}^{1}\right\|_{L^{2}\left(\mathbb{R}_{+}\right)}+\left\|\langle y\rangle^{n}\right\| u_{p y}^{1} \|_{L^{2}\left(\mathbb{R}_{+}\right)}\right)\left\|\left[u_{y}^{\varepsilon}, v_{y}^{\varepsilon}\right]\right\|_{L^{2}} \\
& \leq \varepsilon^{1 / 2-\kappa} C\left(u_{p}^{1}\right)\left\|\left[u^{\varepsilon}, v^{\varepsilon}\right]\right\|_{X} .
\end{aligned}
$$

Similarly for terms in $R_{2}$, we have

$$
\begin{aligned}
\sqrt{\varepsilon}\left\|\left(u_{p}^{1}+\varepsilon^{\gamma} u^{\varepsilon}\right) v_{x}^{\varepsilon}\right\|_{L^{2}} & \leq \sqrt{\varepsilon}\left(\left\|u_{p}^{1}\right\|_{\infty}+\varepsilon^{\gamma}\left\|u^{\varepsilon}\right\|_{\infty}\right)\left\|v_{x}^{\varepsilon}\right\|_{L^{2}} \\
& \leq \varepsilon^{-\kappa}\left(C\left(u_{p}^{1}\right)+\varepsilon^{\frac{\gamma}{2}}\left\|\left[u^{\varepsilon}, v^{\varepsilon}\right]\right\|_{X}\right)\left\|\left[u^{\varepsilon}, v^{\varepsilon}\right]\right\|_{X} \\
\sqrt{\varepsilon}\left\|\left(v_{p}^{1}+\varepsilon^{\gamma} v^{\varepsilon}\right) v_{y}^{\varepsilon}\right\|_{L^{2}} & \leq \sqrt{\varepsilon}\left(\left\|v_{p}^{1}\right\|_{\infty}+\varepsilon^{\gamma}\left\|v^{\varepsilon}\right\|_{\infty}\right)\left\|v_{y}^{\varepsilon}\right\|_{L^{2}} \\
& \leq \varepsilon^{1 / 2-\kappa}\left(C\left(v_{p}^{1}\right)+\varepsilon^{\frac{\gamma}{2}}\left\|\left[u^{\varepsilon}, v^{\varepsilon}\right]\right\|_{X}\right)\left\|\left[u^{\varepsilon}, v^{\varepsilon}\right]\right\|_{X}
\end{aligned}
$$

and

$$
\begin{aligned}
\sqrt{\varepsilon}\left\|u^{\varepsilon} v_{p x}^{1}+v^{\varepsilon} v_{p y}^{1}\right\|_{L^{2}} & \leq \sqrt{\varepsilon}\left(\left\|u^{\varepsilon}\right\|_{\infty}\left\|v_{p x}^{1}\right\|_{L^{2}}+\sup _{x}\left\|\langle y\rangle^{n}\right\| v_{p y}^{1}\left\|_{L^{2}\left(\mathbb{R}_{+}\right)}\right\| v_{y}^{\varepsilon} \|_{L^{2}}\right) \\
& \leq C\left(v_{p}^{1}\right) \varepsilon^{1 / 4-\frac{\gamma}{2}-\kappa}\left\|\left[u^{\varepsilon}, v^{\varepsilon}\right]\right\|_{X},
\end{aligned}
$$

in which we have used the bound (2.57): $\left\|v_{p x}^{1}\right\|_{L^{2}} \lesssim \varepsilon^{-1 / 4-\kappa}$.

Combining the above estimates into (5.6) yields

$$
\begin{aligned}
& \left\|R_{1}\left(u^{\varepsilon}, v^{\varepsilon}\right)\right\|_{L^{2}}+\sqrt{\varepsilon}\left\|R_{2}\left(u^{\varepsilon}, v^{\varepsilon}\right)\right\|_{L^{2}} \\
& \quad \leq C\left(L, u_{s}, v_{s}\right) \varepsilon^{-\gamma-\kappa+\frac{1}{4}}+\varepsilon^{-\frac{\gamma}{2}+\frac{1}{2}}\left\{\left\|\left[u^{\varepsilon}, v^{\varepsilon}\right]\right\|_{X}+\left\|\left[u^{\varepsilon}, v^{\varepsilon}\right]\right\|_{X}^{2}\right\},
\end{aligned}
$$

upon noting that $\gamma+\kappa \leq \frac{1}{4}$ and $\varepsilon \ll 1$, and hence the bound on the gradient of $\bar{u}^{\varepsilon}, \bar{v}^{\varepsilon}$ by (5.6). It remains to bound the sup norm. We now use the estimates on the Stokes problem from Lemma 4.1 with

$$
\begin{aligned}
& f=-u_{s} u_{x}^{\varepsilon}-u_{s x} u^{\varepsilon}-v_{s} u_{y}^{\varepsilon}-v^{\varepsilon} u_{s y}+R_{1} \\
& g=-u_{s} v_{x}^{\varepsilon}-u^{\varepsilon} v_{s x}-v_{s} v_{y}^{\varepsilon}-v^{\varepsilon} v_{s y}+R_{2} .
\end{aligned}
$$

The Stokes estimates, together with (5.6), yield

$$
\begin{aligned}
\varepsilon^{\frac{\gamma}{2}}\left\|\bar{u}^{\varepsilon}\right\|_{\infty}+\varepsilon^{\frac{1}{2}+\frac{\gamma}{2}}\left\|\bar{v}^{\varepsilon}\right\|_{\infty} \leq & C_{\gamma} \varepsilon^{\frac{\gamma}{4}}\left\{\left\|\nabla_{\varepsilon} \bar{u}^{\varepsilon}\right\|_{L^{2}}+\left\|\nabla_{\varepsilon} \bar{v}^{\varepsilon}\right\|_{L^{2}}\right\} \\
& +\varepsilon^{\frac{\gamma}{4}}\left\|-u_{s} u_{x}^{\varepsilon}-u_{s x} u^{\varepsilon}-v_{s} u_{y}^{\varepsilon}-v^{\varepsilon} u_{s y}+R_{1}\right\|_{L^{2}} \\
& +\varepsilon^{\frac{\gamma}{4}}+\frac{1}{2}\left\|-u_{s} v_{x}^{\varepsilon}-u^{\varepsilon} v_{s x}-v_{s} v_{y}^{\varepsilon}-v^{\varepsilon} v_{s y}+R_{2}\right\|_{L^{2}} .
\end{aligned}
$$


The estimates (5.6) and (5.7) have yielded the desired estimates on $\nabla_{\varepsilon}\left[\bar{u}^{\varepsilon}, \bar{v}^{\varepsilon}\right]$ and on $R_{1}, R_{2}$. It remains to give estimates on terms on the right-hand side of the above that involve $\left[u_{s}, v_{s}\right]$. Indeed, since $\left[u_{s}, v_{s}\right]$ are nearly bounded, we have

$$
\begin{aligned}
\varepsilon^{\frac{\gamma}{4}}\left\|u_{s} u_{x}^{\varepsilon}+v_{s} u_{y}^{\varepsilon}\right\|_{L^{2}} & \leq \varepsilon^{\frac{\gamma}{4}-\kappa} C\left(L, u_{s}, v_{s}\right)\left(\left\|u_{x}^{\varepsilon}\right\|_{L^{2}}+\left\|u_{y}^{\varepsilon}\right\|_{L^{2}}\right) \leq \varepsilon^{\frac{\gamma}{4}} C\left(u_{s}, v_{s}\right) \|\left[u^{\varepsilon}, v^{\varepsilon} \|_{X}\right. \\
\varepsilon^{\frac{\gamma}{4}}\left\|u_{s x} u^{\varepsilon}+v^{\varepsilon} u_{s y}\right\|_{L^{2}} & \leq \varepsilon^{\frac{\gamma}{4}}\left(\sup _{x}\left\|\sqrt{y} u_{s x}\right\|_{L^{2}\left(\mathbb{R}_{+}\right)}+\sup _{x}\left\|\sqrt{y} u_{s y}\right\|_{L^{2}\left(\mathbb{R}_{+}\right)}\right)\left\|\left[u_{y}^{\varepsilon}, v_{y}^{\varepsilon}\right]\right\|_{L^{2}} \\
& \leq \varepsilon^{\frac{\gamma}{4}-\kappa} C\left(L, u_{s}, v_{s}\right) \|\left[u^{\varepsilon}, v^{\varepsilon} \|_{X},\right.
\end{aligned}
$$

in which the last inequality used the estimates (3.8) and the following estimate:

$$
\begin{aligned}
\sup _{x} \int y\left|u_{s x}\right|^{2} d y & \leq \sup _{x} \int y\left[\left|u_{p x}^{0}\right|^{2}+\varepsilon\left|u_{e x}^{1}\right|^{2}\right] d y \\
& \leq C\left(u_{p}^{0}\right)+\sup _{x} \int z\left|v_{e z}^{1}\right|^{2} d z \\
& \leq C\left(u_{p}^{0}\right)+L^{-1}\left(\iint z\left|v_{e z}^{1}\right|^{2} d x d z+\iint z\left|v_{e x z}^{1}\right|^{2} d x d z\right) \\
& \leq C\left(L, u_{s}, v_{s}\right) .
\end{aligned}
$$

Similarly, we estimate

$$
\begin{aligned}
\varepsilon^{\frac{\gamma}{4}+\frac{1}{2}}\left\|u_{s} v_{x}^{\varepsilon}+v_{s} v_{y}^{\varepsilon}\right\|_{L^{2}} & \leq \varepsilon^{\frac{\gamma}{4}+\frac{1}{2}-\kappa} C\left(L, u_{s}, v_{s}\right)\left(\left\|v_{x}^{\varepsilon}\right\|_{L^{2}}+\left\|v_{y}^{\varepsilon}\right\|_{L^{2}}\right) \leq \varepsilon^{\frac{\gamma}{4}} C\left(u_{s}, v_{s}\right) \|\left[u^{\varepsilon}, v^{\varepsilon} \|_{X}\right. \\
\varepsilon^{\frac{\gamma}{4}+\frac{1}{2}}\left\|v_{s x} u^{\varepsilon}+v^{\varepsilon} v_{s y}\right\|_{L^{2}} & \leq \varepsilon^{\frac{\gamma}{4}+\frac{1}{2}} \sup _{x}\left(\left\|\sqrt{y} v_{s x}\right\|_{L^{2}}+\left\|\sqrt{y} u_{s x}\right\|_{L^{2}\left(\mathbb{R}_{+}\right)}\right)\left\|\left[u_{y}^{\varepsilon}, v_{y}^{\varepsilon}\right]\right\|_{L^{2}} \\
& \leq \varepsilon^{\frac{\gamma}{4}-\kappa} C\left(L, u_{s}, v_{s}\right) \|\left[u^{\varepsilon}, v^{\varepsilon} \|_{X},\right.
\end{aligned}
$$

in which we have used

$$
\begin{aligned}
\sup _{x} \int y\left|v_{s x}\right|^{2} d y & \leq \sup _{x} \int y\left[\left|v_{p x}^{0}\right|^{2}+\left|v_{e x}^{1}\right|^{2}\right] d y \\
& \leq C\left(v_{p}^{0}\right)+\varepsilon^{-1} \sup _{x} \int z\left|v_{e x}^{1}\right|^{2} d z \\
& \leq C\left(u_{p}^{0}\right)+\varepsilon^{-1} L^{-1}\left(\iint z\left|v_{e x}^{1}\right|^{2} d x d z+\iint z\left|v_{e x x}^{1}\right|^{2} d x d z\right) \\
& \leq \varepsilon^{-1} C\left(L, u_{s}, v_{s}\right) .
\end{aligned}
$$

Hence, putting the above estimates together into the sup estimate (5.8), we have proved

$$
\begin{gathered}
\varepsilon^{\frac{\gamma}{2}}\left\|\bar{u}^{\varepsilon}\right\|_{\infty}+\varepsilon^{\frac{1}{2}+\frac{\gamma}{2}}\left\|\bar{v}^{\varepsilon}\right\|_{\infty} \leq C \\
C\left(u_{s}, v_{s}\right) \varepsilon^{-\frac{3 \gamma}{4}+\frac{1}{4}-\kappa}+\varepsilon^{-\frac{\gamma}{4}+\frac{1}{2}-\kappa}\left\{\left\|\left[u^{\varepsilon}, v^{\varepsilon}\right]\right\|_{X}+\left\|\left[u^{\varepsilon}, v^{\varepsilon}\right]\right\|_{X}^{2}\right\} \\
+\varepsilon^{\frac{\gamma}{4}-\kappa} C\left(L, u_{s}, v_{s}\right) \|\left[u^{\varepsilon}, v^{\varepsilon} \|_{X} .\right.
\end{gathered}
$$

Thus, by definition and the assumption that $\gamma+\kappa \leq \frac{1}{4}$, the estimates (5.7) and (5.9) yield

$$
\left\|\left[\bar{u}^{\varepsilon}, \bar{v}^{\varepsilon}\right]\right\|_{X} \leq C\left(u_{s}, v_{s}\right)\left(1+\varepsilon^{\frac{\gamma}{4}-\kappa} C(L)\left\|\left[u^{\varepsilon}, v^{\varepsilon}\right]\right\|_{X}+\varepsilon^{\frac{3}{8}}\left\|\left[u^{\varepsilon}, v^{\varepsilon}\right]\right\|_{X}^{2}\right) .
$$


This proves that the operator $\left[u^{\varepsilon}, v^{\varepsilon}\right] \mapsto\left[\bar{u}^{\varepsilon}, \bar{v}^{\varepsilon}\right]$ via the problem (5.5) maps the ball $\left\{\left\|\left[u^{\varepsilon}, v^{\varepsilon}\right]\right\|_{X} \leq\right.$ $\left.2 C\left(u_{s}, v_{s}\right)\right\}$ in $X$ into itself, for sufficiently small $\varepsilon$. Moreover, it follows in the similar lines of estimates that

$$
\left\|\left[\bar{u}_{1}^{\varepsilon}-\bar{u}_{2}^{\varepsilon}, \bar{v}_{1}^{\varepsilon}-\bar{v}_{2}^{\varepsilon}\right]\right\|_{X} \leq C\left(L, u_{s}, v_{s}\right) \varepsilon^{\frac{\gamma}{4}-\kappa}\left\{\left\|\left[u_{1}^{\varepsilon}, v_{1}^{\varepsilon}\right]\right\|_{X}+\left\|\left[u_{2}^{\varepsilon}, v_{2}^{\varepsilon}\right]\right\|_{X}\right\}\left\|\left[u_{1}^{\varepsilon}-u_{2}^{\varepsilon}, v_{1}^{\varepsilon}-v_{2}^{\varepsilon}\right]\right\|_{X},
$$

for every two pairs $\left[u_{1}^{\varepsilon}, v_{1}^{\varepsilon}\right]$ and $\left[u_{2}^{\varepsilon}, v_{2}^{\varepsilon}\right]$ in $X$. This proves the existence of the solution to (5.5) with $\left[\bar{u}^{\varepsilon}, \bar{v}^{\varepsilon}\right]=\left[u^{\varepsilon}, v^{\varepsilon}\right]$ via the standard contraction mapping theorem, for sufficiently small $\varepsilon$. The main theorem is proved.

Acknowledgement: The authors wish to thank H. Dong, J. Guzman, and I. Tice for their discussions and pointing out references 2, 13, 14 on regularity of Stokes problems in a domain with corners. Y. Guo's research is supported in part by NSFC grant 10828103 and NSF grant DMS0905255, and T. Nguyen's research was supported in part by the NSF under grant DMS-1405728.

\section{References}

[1] A. Asano, Zero-viscosity Limit of the Incompressible Navier-Stokes Equations, Conference at the 4th Workshop on Mathematical Aspects of Fluid and Plasma dynamics, Kyoto, 1991.

[2] Blum, H. and Rannacher, R.: On the boundary value problem of the biharmonic operator on domains with augular corners. Math. Mech. in the Appl. Sci. 2, 556-581 (1980)

[3] E. Di Nezza, G. Palatucci, E. Valdinoci, Hitchhiker's guide to the fractional Sobolev spaces, Bulletin des Sciences MathZ̆matiques, 136 (2012), pp. 521-573.

[4] L.C. Evans, Partial differential equations. Second edition. Graduate Studies in Mathematics. American Mathematical Society, Providence, RI, 2010. xxii+749 pp.

[5] E, W., and Engquist, B. Blowup of solutions of the unsteady Prandtl's equation. Comm. Pure Appl. Math. 50, 12 (1997), 1287-1293.

[6] D. Gérard-Varet and E. Dormy, On the ill-posedness of the Prandtl equation, J. Amer. Math. Soc., 23 (2010), no. 2, 591-609.

[7] Grenier, E. On the nonlinear instability of Euler and Prandtl equations. Comm. Pure Appl. Math. 53, 9 (2000), 1067-1091.

[8] E. Grenier, Y. Guo, and T. Nguyen. Spectral instability of characteristic boundary layer flows, arXiv:1406.3862.

[9] Y. Guo and T. Nguyen. A note on the Prandtl boundary layers. Comm. Pure Appl. Math. , 64 (2011), no. 10, 1416-1438.

[10] Y. Maekawa, On the inviscid limit problem of the vorticity equations for viscous incompressible flows in the half-plane, Comm. Pure Appl. Math. 67, (2014) 1045-1128.

[11] A. Mazzucato and M. Taylor. Vanishing viscosity plane parallel channel flow and related singular perturbation problems. Anal. PDE, 1(1):35-93, 2008. 
[12] Oleinik, O. A. ; Samokhin, V. N. Mathematical models in boundary layer theory. Applied Mathematics and Mathematical Computation, 15. Chapman \& Hall/CRC, Boca Raton, FL, 1999. $\mathrm{x}+516 \mathrm{pp}$.

[13] M. Orlt, Regularity for Navier-Stokes in domains with corners, PhD Thesis, 1998 (in German).

[14] M. Orlt and A.-M. Sändig, Regularity of viscous Navier-Stokes flows in nonsmooth domains. Boundary value problems and integral equations in nonsmooth domains (Luminy, 1993), 185201, Lecture Notes in Pure and Appl. Math., 167, Dekker, New York, 1995.

[15] M. Sammartino and R. Caflisch, Zero viscosity limit for analytic solutions of the Navier-Stokes equation on a half-space. I. Existence for Euler and Prandtl equations. Comm. Math. Phys. 192 (1998), no. 2, 433-461.

[16] M. Sammartino and R. Caflisch, Zero viscosity limit for analytic solutions, of the NavierStokes equation on a half-space. II. Construction of the Navier-Stokes solution. Comm. Math. Phys. 192 (1998), no. 2, 463-491.

[17] Schlichting, H and Gersten, K.: Boundary layer theory. 8th Edition, Springer-Verlag 2000. 